\title{
Neutrino oscillograms of the Earth: effects of 1-2 mixing and CP-violation
}

\author{
Evgeny Kh. Akhmedov \\ Max-Planck-Institut für Kernphysik, Postfach 103980, D-69029 Heidelberg, Germany \\ and: National Research Centre Kurchatov Institute, Moscow, Russia \\ E-mail: akhmedov@mpi-hd.mpg.de

\section{Michele Maltoni} \\ Departamento de Física Teórica 6 Instituto de Física Teórica UAM/CSIC, Facultad de \\ Ciencias C-XI, Universidad Autónoma de Madrid, Cantoblanco, E-28049 Madrid, Spain \\ E-mail: michele.maltoni@uam.es
}

\author{
Alexei Yu. Smirnov \\ The Abdus Salam International Centre for Theoretical Physics, Strada Costiera 11, \\ I-34014 Trieste, Italy \\ and: Institute for Nuclear Research, Russian Academy of Sciences, Moscow, Russia \\ E-mail: smirnov@ictp.trieste.it
}

\begin{abstract}
We develop a comprehensive description of three flavor neutrino oscillations inside the Earth in terms of neutrino oscillograms in the whole range of nadir angles and for energies above $0.1 \mathrm{GeV}$. The effects of the 1-2 mass splitting and mixing as well the interference of the 1-2 and 1-3 modes of oscillations are quantified. The 1-2 mass splitting and mixing lead to the appearance, apart from the resonance MSW peaks, of the parametric resonance peak for core-crossing trajectories at $E_{\nu} \sim 0.2 \mathrm{GeV}$. We show that the interference effects, in particular $\mathrm{CP}$ violation, have a domain structure with borders determined by the solar and atmospheric magic lines and the lines of the interference phase condition. The dependence of the oscillograms on the Dirac CP-violating phase is studied. We show that for $\sin ^{2} 2 \theta_{13}<0.1$ the strongest dependence of the oscillograms on $\delta$ is in the 1-2 and 1-3 resonance regions.
\end{abstract}

KEYWORDS: neutrino oscillations, matter effects, leptonic CP violation. 


\section{Contents}

1. Introduction 1

2. Three-flavor neutrino oscillations in matter 3

2.1 Evolution matrix and probabilities for symmetric profile 3

2.2 Eigenvalues 5

$\begin{array}{ll}2.3 & \text { Amplitudes in medium of constant density }\end{array}$

$\begin{array}{lll}2.4 & \text { The factorization approximation } & 8\end{array}$

3. Effects of 1-2 splitting and mixing $\quad 9$

3.1 Neutrino oscillograms of the Earth 9

$\begin{array}{ll}3.2 \text { Oscillograms due to 1-2 mixing } & 10\end{array}$

$\begin{array}{lll}3.3 & \nu_{e}-\nu_{e} \text { channel } & 15\end{array}$

$\begin{array}{lll}3.4 & \nu_{e}-\nu_{\mu} \text { and } \nu_{e}-\nu_{\tau} \text { channels } & 19\end{array}$

$3.5 \nu_{\mu}-\nu_{\mu}$ and $\nu_{\tau}-\nu_{\tau}$ channels 22

$\begin{array}{lll}3.6 & \nu_{\mu}-\nu_{\tau} \text { channel } & 25\end{array}$

$\begin{array}{ll}3.7 & \text { Inverted mass hierarchy } \\ \end{array}$

4. Effects of CP-violating phase $\delta \quad \mathbf{2 8}$

$\begin{array}{lll}4.1 & \text { Interference and CP-violation } & 28\end{array}$

4.2 "Magic" lines and interference phase lines 30

$\begin{array}{lll}4.3 & \text { CP-phase domains for channels involving } \nu_{e} & 36\end{array}$

$\begin{array}{lll}4.4 & \text { CP-domains for channels not involving } \nu_{e} & 36\end{array}$

4.5 Sensitivity to the CP phase in the $\nu_{\mu}-\nu_{e}$ channel 38

4.6 Sensitivity to the CP phase in the $\nu_{\mu}-\nu_{\mu}$ channel 42

5. Discussion and conclusions $\quad 43$

\section{Introduction}

Substantial future progress in neutrino physics will be related to the long baseline experiments as well as studies of the cosmic and atmospheric neutrinos. These studies are expected to fill some of the outstanding gaps in our knowledge of neutrino properties, such as the value of the leptonic mixing angle $\theta_{13}$, the type of the neutrino mass hierarchy, the octant of the mixing angle $\theta_{23}$ and the size of the Dirac-type leptonic CP-violation. They are also expected to improve the accuracy of the determination of the already known parameters, such as the mass squared differences $\Delta m_{21}^{2}$ and $\left|\Delta m_{31}^{2}\right|$ and the mixing parameters $\theta_{12}$ and $\sin ^{2} 2 \theta_{23}$. 
The key element of these experiments is that neutrinos propagate long distances inside the Earth before reaching detectors, and therefore a careful analysis of the Earth matter effects on neutrino oscillations is necessary (see, e.g., references in $[1,2]$ as well as Refs. [3, $4,5,6,7,8,9,10,11,12,13,14,15,16,17,18,19,20,21])$. In a previous publication [1] we have studied these matter effects by making use of "neutrino oscillograms" of the Earth as the main tool. These are the contours of constant oscillation probabilities in the plane of neutrino energy and nadir angle. The plots of this type were introduced by P. Lipari in 1998 (unpublished) and then appeared in several publications [22, 23, 24, 25, 1]. The oscillograms exhibit a very rich structure with local and global maxima and minima, including the MSW $[26,27]$ resonance maxima in the mantle and core of the Earth and the parametric enhancement $[28,29,4,5,30,31,32,22,33]$ ridges for core-crossing neutrino trajectories. It was shown in [1] that all these features, including the local and global minima and maxima as well as saddle points can be understood in terms of various realizations of just two conditions: the generalized amplitude and phase conditions. We refer the reader to Ref. [1] for details. It has been shown that these oscillograms are very useful for gaining an insight into the physics of neutrino oscillations in the Earth and should help plan the future experiments as well as interpret their data.

The analysis in [1] was performed in the limit of vanishing "solar" mass squared splitting $\Delta m_{21}^{2}$. While this approximation is quantitatively well justified at relatively high neutrino energies $\left(E_{\nu} \gtrsim 3 \mathrm{GeV}\right)$, it is less satisfactory at lower energies and also misses some important 3 -flavor features of neutrino oscillations, most notably $\mathrm{CP}$ violation. In the present paper we extend the study of [1] to the case $\Delta m_{21}^{2} \neq 0$ and consider 3-flavor effects in neutrino oscillations in the Earth, with the emphasis on the effects of the CP violating phase $\delta$. We explore in detail

- the effects of the 1-2 mixing and splitting on the oscillation probabilities,

- the interference of the 1-2 and 1-3 (i.e., "solar" and "atmospheric") amplitudes,

- the effects of and the sensitivity to the Dirac-type CP-violating phase $\delta$,

- the dependence of the oscillation probabilities on the neutrino mass hierarchy.

We perform numerical calculations of oscillation probabilities and also develop a simple analytic approach to interpretation of the obtained results based on lines of three types in the neutrino energy - nadir angle plane. These are the solar and atmospheric "magic lines", i.e., the lines on which respectively the solar or atmospheric contributions to the transition amplitude approximately vanish, and the interference phase lines. Construction of these curves allows one to identify the regions in the experimental parameter space that are most sensitive to the effects of non-vanishing phase $\delta$. Our results can be useful for planning experiments with atmospheric and accelerator neutrinos, as well as neutrinos of cosmic origin.

In the present paper, as well as in [1], we confine our consideration to the study of the oscillations probabilities. Accurate predictions for the event numbers and sensitivities of future experiments can only be done when an information on the corresponding detection efficiencies and systematic errors becomes available. Still, some general statements can be 
made and estimates done even in the absence of such an information; we plan to present the corresponding analysis in a future publication.

Three-flavor effects in neutrino oscillations in the Earth have been considered in the past $[34,35,36,7,23,37,38,39,40,41,11,42,43,44,45,24,46,47,48,17,49,50,51$, $52,53,54,55]$. The main new results of the present paper are the analysis of the neutrino oscillations in the Earth in terms of the aforementioned three sets of curves, and use of the neutrino oscillograms for a detailed study of the domains of the parameter space that are most sensitive to the value of CP-violating phase $\delta$.

The paper is organized as follows. In Sec. 2 we give general 3-flavor expressions for the oscillation probabilities in matter. We present exact analytic results for matter with constant density and introduce a factorization approximation. In Sec. 3 present the neutrino oscillograms for different oscillation channels and discuss the effects of non-vanishing mixing and splitting in the 1-2 sector on these oscillograms. We consider features of the oscillograms for the inverted mass hierarchy. Sec. 4 contains the discussion of the effects of the CP-violating phase $\delta$ and their analysis in terms of the three sets of special lines. In this section we also discuss the sensitivity of the oscillation probabilities to the phase $\delta$ and its dependence on neutrino energy and nadir angle (baseline length). Conclusions follow in Sec. 5.

\section{Three-flavor neutrino oscillations in matter}

\subsection{Evolution matrix and probabilities for symmetric profile}

We consider mixing of the three flavor neutrinos, $\nu_{f} \equiv\left(\nu_{e}, \nu_{\mu}, \nu_{\tau}\right)^{T}$. The mixing matrix $U$, defined through $\nu_{f}=U \nu_{m}$, where $\nu_{m}=\left(\nu_{1}, \nu_{2}, \nu_{3}\right)^{T}$ is the vector of neutrino mass eigenstates, can be parametrized as

$$
U=U_{23} I_{\delta} U_{13} I_{-\delta} U_{12} .
$$

Here the matrices $U_{i j}=U_{i j}\left(\theta_{i j}\right)$ describe rotations in the $i j$-planes by the angles $\theta_{i j}$, and $I_{\delta} \equiv \operatorname{diag}\left(1,1, e^{i \delta}\right)$, where $\delta$ is the Dirac-type CP-violating phase.

Evolution of the system in matter is described by the equation

$$
i \frac{d \nu_{f}}{d t}=\left(\frac{U M^{2} U^{\dagger}}{2 E_{\nu}}+\hat{V}\right) \nu_{f},
$$

where $E_{\nu}$ is the neutrino energy and $M^{2} \equiv \operatorname{diag}\left(0, \Delta m_{21}^{2}, \Delta m_{31}^{2}\right)$ is the diagonal matrix of neutrino mass squared differences with $\Delta m_{j i}^{2} \equiv m_{j}^{2}-m_{i}^{2} . \hat{V}=\operatorname{diag}\left(V_{e}, 0,0\right)$ is the matrix of matter-induced neutrino potentials with $V_{e} \equiv \sqrt{2} G_{F} N_{e}, G_{F}$ and $N_{e}$ being the Fermi constant and the electron number density, respectively. The evolution matrix $S\left(t, t_{0}\right)$ (the matrix of oscillation amplitudes) defined through $\nu(t)=S\left(t, t_{0}\right) \nu\left(t_{0}\right)$ satisfies the same Eq. (2.2) with the initial condition $S\left(t_{0}, t_{0}\right)=\mathbb{1}$.

It is convenient to consider the evolution of the neutrino system in the propagation basis $\tilde{\nu}=\left(\nu_{e}, \tilde{\nu}_{2}, \tilde{\nu}_{3}\right)^{T}$ defined through the relation

$$
\nu_{f}=U_{23} I_{\delta} \tilde{\nu}
$$


with $\tilde{\nu}=U_{13} I_{-\delta} U_{12} \nu$. According to Eqs. (2.2) and (2.1), the Hamiltonian $\tilde{H}$ which describes the evolution of the neutrino vector of state $\tilde{\nu}$ is

$$
\tilde{H}=\frac{1}{2 E_{\nu}} U_{13} U_{12} M^{2} U_{12}^{\dagger} U_{13}^{\dagger}+\hat{V} .
$$

This Hamiltonian does not depend on the 2-3 mixing and the CP-violating phase. The dependence on these parameters appears when one projects the initial flavor state on the propagation basis and the final state back onto the original flavor basis. Explicitly, the Hamiltonian $\tilde{H}$ reads

$$
\tilde{H}=\frac{\Delta m_{31}^{2}}{2 E_{\nu}}\left(\begin{array}{ccc}
s_{13}^{2}+s_{12}^{2} c_{13}^{2} r_{\Delta}+2 V_{e} E_{\nu} / \Delta m_{31}^{2} & s_{12} c_{12} c_{13} r_{\Delta} & s_{13} c_{13}\left(1-s_{12}^{2} r_{\Delta}\right) \\
\ldots & c_{12}^{2} r_{\Delta} & -s_{12} c_{12} s_{13} r_{\Delta} \\
\ldots & \ldots & c_{13}^{2}+s_{12}^{2} s_{13}^{2} r_{\Delta}
\end{array}\right) .
$$

Here $r_{\Delta} \equiv \Delta m_{21}^{2} / \Delta m_{31}^{2}, c_{i j} \equiv \cos \theta_{i j}$ and $s_{i j} \equiv \sin \theta_{i j}$. We introduce the evolution matrix (the matrix of transition and survival amplitudes) in the basis $\left(\nu_{e}, \tilde{\nu}_{2}, \tilde{\nu}_{3}\right)$ as

$$
\tilde{S}=\left(\begin{array}{lll}
A_{e e} & A_{e \tilde{2}} & A_{e \tilde{3}} \\
A_{\tilde{2} e} & A_{\tilde{2} \tilde{2}} & A_{\tilde{2} \tilde{3}} \\
A_{\tilde{3} e} & A_{\tilde{3} \tilde{2}} & A_{\tilde{3} \tilde{3}}
\end{array}\right)
$$

This matrix satisfies the Schrödinger equation with the Hamiltonian $\tilde{H}$. Then, according to Eq. (2.3), the evolution matrix in the flavor basis $S$ is

$$
S=\tilde{U} \tilde{S} \tilde{U}^{\dagger}, \quad \tilde{U} \equiv U_{23} I_{\delta}
$$

As follows immediately from the form of the Hamiltonian in Eq. (2.5), the amplitudes have the following hierarchy:

$$
A_{e \tilde{2}}, A_{\tilde{2} e} \sim r_{\Delta}, \quad A_{e \tilde{3}}, A_{\tilde{3} e} \sim s_{13}, \quad A_{\tilde{3} \tilde{2}}, A_{\tilde{2} \tilde{3}} \sim s_{13} r_{\Delta}
$$

i.e., $A_{\tilde{2} \tilde{3}}$ and $A_{\tilde{3} \tilde{2}}$ are the smallest amplitudes.

In terms of the propagation-basis amplitudes, according to Eqs. (2.6) and (2.7) (see also [43]), the matrix $S$ is given by

$$
S=\left(\begin{array}{ccc}
A_{e e} & c_{23} A_{e \tilde{2}}+s_{23} e^{-i \delta} A_{e \tilde{3}} & -s_{23} A_{e \tilde{2}}+c_{23} e^{-i \delta} A_{e \tilde{3}} \\
c_{23} A_{\tilde{2} e}+s_{23} e^{i \delta} A_{\tilde{3} e} & c_{23}^{2} A_{\tilde{2} \tilde{2}}+s_{23}^{2} A_{\tilde{3} \tilde{3}}+K_{\mu \mu} & -s_{23} c_{23}\left(A_{\tilde{2} \tilde{2}}-A_{\tilde{3} \tilde{3}}\right)+K_{\mu \tau} \\
-s_{23} A_{\tilde{2} e}+c_{23} e^{i \delta} A_{\tilde{3} e}-s_{23} c_{23}\left(A_{\tilde{2} \tilde{2}}-A_{\tilde{3} \tilde{3}}\right)+K_{\tau \mu} & s_{23}^{2} A_{\tilde{2} \tilde{2}}+c_{23}^{2} A_{\tilde{3} \tilde{3}}+K_{\tau \tau}
\end{array}\right)
$$

where

$$
\begin{aligned}
K_{\mu \mu} & \equiv s_{23} c_{23}\left(e^{-i \delta} A_{\tilde{2} \tilde{3}}+e^{i \delta} A_{\tilde{3} \tilde{2}}\right) \\
K_{\mu \tau} & \equiv c_{23}^{2} e^{-i \delta} A_{\tilde{2} \tilde{3}}-s_{23}^{2} e^{i \delta} A_{\tilde{3} \tilde{2}} \\
K_{\tau \mu} & =K_{\mu \tau}(\delta \rightarrow-\delta, \tilde{2} \leftrightarrow \tilde{3}) \\
K_{\tau \tau} & =-K_{\mu \mu}
\end{aligned}
$$

Notice that $K_{\alpha \beta}(\alpha, \beta=\mu, \tau)$ are proportional to the small amplitudes $A_{\tilde{2} \tilde{3}}$ and $A_{\tilde{3} \tilde{2}}$. 
Since the Earth density profile is to a good approximation symmetric with respect to the midpoint of the neutrino trajectory and there is no fundamental CP- (and T-) violation in the propagation basis, the neutrino evolution is T-invariant in this basis, which yields [11]

$$
A_{\tilde{2} e}=A_{e \tilde{2}}, \quad A_{\tilde{3} e}=A_{e \tilde{3}}, \quad A_{\tilde{3} \tilde{2}}=A_{\tilde{2} \tilde{3}} .
$$

Therefore for $K_{\alpha \beta}$ we obtain

$$
\begin{aligned}
& K_{\mu \tau}=A_{\tilde{2} \tilde{3}}\left(\cos 2 \theta_{23} \cos \delta-i \sin \delta\right), \\
& K_{\tau \mu}=A_{\tilde{2} \tilde{3}}\left(\cos 2 \theta_{23} \cos \delta+i \sin \delta\right), \\
& K_{\mu \mu}=-K_{\tau \tau}=A_{\tilde{2} \tilde{3}} \sin 2 \theta_{23} \cos \delta .
\end{aligned}
$$

Notice that the diagonal elements $S_{\mu \mu}$ and $S_{\tau \tau}$ of the evolution matrix (2.9) depend on the CP phase only via $\cos \delta$, whereas $S_{e e}$ does not depend on $\delta$ at all. The latter is a consequence of our use of the standard parametrization (2.1) for the leptonic mixing matrix.

The oscillation probabilities are expressed through the matrix elements of $S$ as

$$
P_{\alpha \beta} \equiv P\left(\nu_{\alpha} \rightarrow \nu_{\beta}\right)=\left|S_{\beta \alpha}\right|^{2} \quad \text { with } \quad \alpha, \beta=e, \mu, \tau .
$$

From Eqs. (2.13), (2.9), (2.11) and (2.12) one finds for the probabilities $P_{\alpha \beta}$

$$
\begin{aligned}
& P_{\mu e}=c_{23}^{2}\left|A_{e \tilde{2}}\right|^{2}+s_{23}^{2}\left|A_{e \tilde{3}}\right|^{2}+2 s_{23} c_{23} \operatorname{Re}\left(e^{-i \delta} A_{e \tilde{2}}^{*} A_{e \tilde{3}}\right), \\
& P_{\tau e}=s_{23}^{2}\left|A_{e \tilde{2}}\right|^{2}+c_{23}^{2}\left|A_{e \tilde{3}}\right|^{2}-2 s_{23} c_{23} \operatorname{Re}\left(e^{-i \delta} A_{e \tilde{2}}^{*} A_{e \tilde{3}}\right), \\
& P_{\mu \mu}=\left|c_{23}^{2} A_{\tilde{2} \tilde{2}}+s_{23}^{2} A_{\tilde{3} \tilde{3}}+2 s_{23} c_{23} \cos \delta A_{\tilde{2} \tilde{3}}\right|^{2}, \\
& P_{\mu \tau}=\left|s_{23} c_{23}\left(A_{\tilde{3} \tilde{3}}-A_{\tilde{2} \tilde{2}}\right)+\left(\cos 2 \theta_{23} \cos \delta+i \sin \delta\right) A_{\tilde{2} \tilde{3}}\right|^{2} .
\end{aligned}
$$

The probabilities $P_{\beta \alpha}$ are obtained from $P_{\alpha \beta}$ through the substitution $\delta \rightarrow-\delta:^{1}$

$$
P_{\beta \alpha}=P_{\alpha \beta}(\delta \rightarrow-\delta) .
$$

All the results presented in this section are also valid for antineutrinos if one makes substitutions

$$
\delta \rightarrow-\delta, \quad A_{i j} \rightarrow \bar{A}_{i j}, \quad \text { where } \quad \bar{A}_{i j} \equiv A_{i j}(V \rightarrow-V) .
$$

Notice that the amplitudes of transitions (2.14) and (2.15) that involve $\nu_{e}$ are given by linear combinations of two propagation-basis amplitudes. The other amplitudes depend on three propagation-basis amplitudes.

\subsection{Eigenvalues}

We will refer to parameters $\theta_{12}$ and $\Delta m_{21}^{2}$ as the 1-2 (or solar) sector, and to parameters $\theta_{13}$ and $\Delta m_{31}^{2}$ as the 1-3 (or atmospheric) sector. We use the same form of the mixing matrix in

\footnotetext{
${ }^{1}$ Note that in a matter with an asymmetric density profile one would also have to substitute $V \rightarrow \tilde{V}$, where $\tilde{V}$ is the reverse profile corresponding to the interchanged positions of the neutrino source and detector [11]). This, in particular, means that one would have to distinguish $A_{i j}$ from $A_{j i}$ and $K_{\beta \alpha}$ from $K_{\alpha \beta}$.
} 
matter as in vacuum, with substitution $\theta_{i j} \rightarrow \theta_{i j}^{m}$. The eigenvalues of the Hamiltonian $H_{i}^{m}$ are identified in such a way that $H_{i}^{m} \rightarrow \Delta m_{i 1}^{2} / 2 E_{\nu}$ when $V \rightarrow 0$. For densities (energies) between the 1-2 and 1-3 MSW resonances we find for the normal mass hierarchy

$$
H_{1}^{m} \approx \frac{\Delta m_{21}^{2} c_{12}^{2}}{2 E_{\nu}}, \quad H_{2}^{m} \approx V, \quad H_{3}^{m} \approx \frac{\Delta m_{31}^{2}}{2 E_{\nu}} .
$$

For high densities (energies) which are far above the 1-3 resonance we have

$$
H_{1}^{m} \approx \frac{\Delta m_{21}^{2} c_{12}^{2}}{2 E_{\nu}}, \quad H_{2}^{m} \approx \frac{\Delta m_{31}^{2} c_{13}^{2}}{2 E_{\nu}}, \quad H_{3}^{m} \approx V .
$$

In a constant density medium the oscillation phases equal

$$
2 \phi_{j i}^{m}=\Delta H_{j i} L \quad \text { with } \quad \Delta H_{j i} \equiv H_{j}^{m}-H_{i}^{m} .
$$

There are two independent frequencies in the problem, $\Delta H_{21}$ and $\Delta H_{32}$.

For antineutrinos there are no level crossings, and with the increase of density (energy) the eigenvalues have the following asymptotic limits:

$$
H_{1}^{m} \rightarrow V, \quad H_{2}^{m} \rightarrow \frac{\Delta m_{21}^{2} c_{12}^{2}}{2 E_{\nu}}, \quad H_{3}^{m} \rightarrow \frac{\Delta m_{31}^{2} c_{13}^{2}}{2 E_{\nu}} .
$$

We will discuss the level crossing scheme for the inverted hierarchy in Sec. 3.7.

\subsection{Amplitudes in medium of constant density}

Oscillations in a matter of constant (but trajectory dependent) density layers give a good approximation to the results of exact numerical calculations for neutrino oscillations in the Earth. As we have shown in [1] they reproduce rather accurately all the features of the oscillograms (at least for $\Delta m_{21}^{2}=0$ ). Thus, we can use the exact analytic results for constant-density matter to clarify various features of the numerical results for the realistic Earth density profile. This also allows one to obtain a parametric dependence of the probabilities, in particular parametric smallness of certain contributions to the probabilities. In what follows we present the results for one layer of constant density. We mark the corresponding amplitudes and probabilities with the superscript "cst".

The exact formula for the $\nu_{\mu} \rightarrow \nu_{e}$ transition amplitude in matter of constant density is

$$
S_{e \mu}^{\mathrm{cst}}=2 i e^{i \phi_{21}^{m}}\left[U_{e 1}^{m} U_{\mu 1}^{m *} \sin \phi_{21}^{m}-e^{-i \phi_{31}^{m}} U_{e 3}^{m} U_{\mu 3}^{m *} \sin \phi_{32}^{m}\right],
$$

where $U_{\alpha j}^{m}$ and $\phi_{j i}^{m}$ are the elements of mixing matrix and the oscillation half-phases in matter. Using the expressions for $U_{e i}^{m}$ and $U_{\mu i}^{m}$ in terms of the mixing angles in the standard parametrization, we can rewrite Eq. (2.24) as

$$
S_{e \mu}^{\mathrm{cst}}=\cos \theta_{23}^{m} A_{e \tilde{2}}^{\mathrm{cst}}+\sin \theta_{23}^{m} e^{-i \delta^{m}} A_{e \tilde{3}}^{\mathrm{cst}},
$$

where

$$
\begin{aligned}
& A_{e \tilde{2}}^{\mathrm{cst}} \equiv-i e^{i \phi_{21}^{m}} \cos \theta_{13}^{m} \sin 2 \theta_{12}^{m} \sin \phi_{21}^{m} \\
& A_{e \tilde{3}}^{\mathrm{css}} \equiv-i e^{i \phi_{21}^{m}} \sin 2 \theta_{13}^{m}\left[\sin \phi_{32}^{m} e^{-i \phi_{31}^{m}}+\cos ^{2} \theta_{12}^{m} \sin \phi_{21}^{m}\right] .
\end{aligned}
$$


Here $\phi_{31}^{m}=\phi_{32}^{m}+\phi_{21}^{m}$. Since to a good approximation $\theta_{23}^{m} \approx \theta_{23}$ and $\delta^{m} \approx \delta[56,42]$, the amplitudes $A_{\tilde{2}}^{\text {cst }}$ and $A_{e \tilde{3}}^{\text {cst }}$ can be identified with $A_{e \tilde{2}}$ and $A_{e \tilde{3}}$ in Eq. (2.14) and (2.15). According to Eq. (2.20), between the two MSW resonances $\phi_{31}^{m} \approx \Delta m_{32}^{2} L / 4 E_{\nu}$. Above the 1-2 resonance $\sin ^{2} \theta_{12}^{m} \approx 1$, so that the second term in (2.27) is very small:

$$
\cos ^{2} \theta_{12}^{m} \approx \frac{\sin ^{2} 2 \theta_{12}}{4}\left(\frac{\Delta m_{21}^{2}}{2 V E_{\nu}}\right)^{2}=\frac{1}{4} \tan ^{2} 2 \theta_{12}\left(\frac{E_{12}^{R}}{E_{\nu}}\right)^{2},
$$

which is suppressed as $1 / V^{2}$. Here $E_{12}^{R} \approx \cos 2 \theta_{12} \Delta m_{21}^{2} / 2 V$ is the 1-2 resonance energy. Consequently, up to the phase factor, the amplitude $A_{e \tilde{3}}$ is reduced to the $2 \nu$ form with the parameters $\left(\theta_{13}^{m}, \phi_{32}^{m}\right)$. Notice that above the 1-3 resonance $\phi_{21}^{m} \rightarrow \Delta m_{32}^{2} L / 4 E_{\nu}$.

Similarly, for the $\nu_{\mu} \rightarrow \nu_{\mu}$ amplitude we obtain

$$
S_{\mu \mu}^{\mathrm{cst}}=1+2 i e^{i \phi_{21}^{m}}\left|U_{\mu 1}^{m}\right|^{2} \sin \phi_{21}^{m}-2 i e^{-i \phi_{32}^{m}}\left|U_{\mu 3}^{m}\right|^{2} \sin \phi_{32}^{m} .
$$

In terms of mixing angles,

$$
U_{\mu 1}^{m}=-s_{12}^{m} c_{23}^{m}-c_{12}^{m} s_{13}^{m} s_{23}^{m} e^{i \delta^{m}}, \quad U_{\mu 3}^{m}=c_{13}^{m} s_{23}^{m},
$$

and the amplitude can be rewritten as

$$
S_{\mu \mu}^{\mathrm{cst}}=\cos ^{2} \theta_{23}^{m} A_{\tilde{2} \tilde{2}}^{\mathrm{cst}}+\sin ^{2} \theta_{23}^{m} A_{\tilde{3} \tilde{3}}^{\mathrm{cst}}+\sin 2 \theta_{23}^{m} \cos \delta^{m} A_{\tilde{2} \tilde{3}}^{\mathrm{cst}},
$$

where

$$
\begin{aligned}
& A_{\tilde{2} \tilde{2}}^{\mathrm{cst}} \equiv 1+2 i e^{i \phi_{21}^{m}} \sin ^{2} \theta_{12}^{m} \sin \phi_{21}^{m}, \\
& A_{\tilde{3} \tilde{3}}^{\mathrm{cst}} \equiv 1-2 i e^{-i \phi_{32}^{m}} \cos ^{2} \theta_{13}^{m} \sin \phi_{32}^{m}+2 i e^{i \phi_{21}^{m}} \sin ^{2} \theta_{13}^{m} \cos ^{2} \theta_{12}^{m} \sin \phi_{21}^{m}, \\
& A_{\tilde{2} \tilde{3}}^{\mathrm{cst}} \equiv i e^{i \phi_{21}^{m}} \sin \theta_{13}^{m} \sin 2 \theta_{12}^{m} \sin \phi_{21}^{m} .
\end{aligned}
$$

Notice that $A_{\tilde{2} \tilde{2}}^{\text {cst }}$ has exactly the form of the corresponding $2 \nu$ amplitude driven by the solar parameters. The amplitude $A_{\tilde{3} \tilde{3}}^{\text {cst }}$ also coincides to a very good approximation with the corresponding $2 \nu$ amplitude driven by the atmospheric parameters. The contribution of the solar mode to $A_{\tilde{3} \tilde{3}}^{\text {cst }}$ is strongly suppressed by the factor $\sin ^{2} \theta_{13}$ in the region of 1-2 resonance and by $\cos ^{2} \theta_{12}^{m}$ above this resonance. Up to the overall factor $\sin \theta_{13}^{m}$ the amplitude $A_{\tilde{2} \tilde{3}}^{\text {cst }}$ depends on the solar (1-2) phase only, and in general it contains double smallness: $\sin \theta_{13}$ and the one related to the solar mode of oscillations. Again in the approximation $\theta_{23}^{m} \approx \theta_{23}$ and $\delta^{m} \approx \delta$ the amplitudes (2.32), (2.33) and (2.34) can be identified with the corresponding amplitudes in the propagation basis.

For completeness, we present also the expression for the $\nu_{e} \rightarrow \nu_{e}$ amplitude:

$$
S_{e e}^{\mathrm{cst}}=1+2 i e^{i \phi_{21}^{m}} \cos ^{2} \theta_{13}^{m} \cos ^{2} \theta_{12}^{m} \sin \phi_{21}^{m}-2 i e^{-i \phi_{32}^{m} \sin ^{2} \theta_{13}^{m} \sin \phi_{32}^{m} .}
$$

It can be rewritten in the form convenient for use at low energies:

$$
S_{e e}^{\mathrm{cst}}=1+2 i e^{i \phi_{21}^{m}} \cos ^{2} \theta_{12}^{m} \sin \phi_{21}^{m}-2 i e^{i \phi_{21}^{m}} \sin ^{2} \theta_{13}^{m}\left[e^{-i \phi_{32}^{m}} \sin \phi_{31}^{m}+\cos ^{2} \theta_{12}^{m} \sin \phi_{21}^{m}\right] \text {. }
$$

In formulas for the amplitudes one can interchange the phases in the exponents and sines using the following identity:

$$
\sin \phi_{32}^{m} e^{-i \phi_{31}^{m}}=\sin \phi_{31}^{m} e^{-i \phi_{32}^{m}}-\sin \phi_{21}^{m},
$$

where $\phi_{31}^{m}=\phi_{32}^{m}+\phi_{21}^{m}$. Due to the level crossing phenomenon this phase interchange is convenient for studies of oscillation effects in different energy ranges. 


\subsection{The factorization approximation}

The elements of the evolution matrix in the propagation basis $\tilde{S}$ depend in general on $\Delta m_{21}^{2}, \theta_{12}, \Delta m_{31}^{2}$ and $\theta_{13}$. As follows immediately from the form of the Hamiltonian $\tilde{H}$ in Eq. (2.5), in the limits $\Delta m_{21}^{2} \rightarrow 0$ or/and $s_{12} \rightarrow 0$ the state $\tilde{\nu}_{2}$ decouples from the rest of the system, and consequently, the amplitude $A_{e \tilde{2}}$ vanishes. In this limit, $A_{e \tilde{3}}$ (as well as $A_{\tilde{3} \tilde{3}}$ and $\left.S_{e e}\right)$ is reduced to a $2 \nu$ amplitude which depends on the parameters $\Delta m_{31}^{2}$ and $\theta_{13}$. We denote the latter by $A_{A}$ :

$$
A_{A}\left(\Delta m_{31}^{2}, \theta_{13}\right) \equiv A_{e \tilde{3}}\left(\Delta m_{21}^{2}=0\right) .
$$

It is this amplitude that has been studied in our previous paper [1]; the corresponding probability equals $P_{A}=\left|A_{A}\right|^{2}$.

In the limit $s_{13} \rightarrow 0$ the state $\tilde{\nu}_{3}$ decouples and the amplitude $A_{e \tilde{3}}$ vanishes. At the same time, the amplitude $A_{e \tilde{2}}$ reduces to a $2 \nu$ amplitude depending on the parameters of the 1-2 sector, $\Delta m_{21}^{2}$ and $\theta_{12}$. Denoting this amplitude by $A_{S}$ we have

$$
A_{S}\left(\Delta m_{21}^{2}, \theta_{12}\right) \equiv A_{e \tilde{2}}\left(\theta_{13}=0\right) \text {. }
$$

We will also use the notation $P_{S} \equiv\left|A_{S}\right|^{2}$.

This consideration implies that to the leading non-trivial order in the small parameters $s_{13}$ and $r_{\Delta}$ the amplitudes $A_{e \tilde{2}}$ and $A_{\tilde{2} e}$ below the 1-3 resonance depend only on the "solar" parameters, whereas the amplitudes $A_{e \tilde{3}}$ and $A_{\tilde{3} e}$ above the 1-2 resonance depend only on the "atmospheric" parameters, i.e.:

$$
\begin{aligned}
& A_{e \tilde{2}} \simeq A_{\tilde{2} e} \simeq A_{S}\left(\Delta m_{21}^{2}, \theta_{12}\right), \quad E_{\nu}<E_{13}^{R}, \\
& A_{e \tilde{3}} \simeq A_{\tilde{3} e} \simeq A_{A}\left(\Delta m_{31}^{2}, \theta_{13}\right), \quad E_{\nu}>E_{12}^{R} \text {. }
\end{aligned}
$$

In what follows we will call the approximate equalities in Eq. (2.40) the factorization approximation, since the dependence of the interference terms in the probabilities, $A_{\tilde{2} e}^{*} A_{\tilde{3} e}$, on solar and atmospheric parameters factorizes in this approximation.

An additional insight into the factorization approximation can be obtained from the results for constant-density matter obtained in the previous section. According to Eqs. (2.26) and (2.27), up to the phase factors one has

$$
A_{A} \rightarrow A_{A}^{\mathrm{cst}} \equiv \sin 2 \theta_{13}^{m} \sin \phi_{A}, \quad A_{S} \rightarrow A_{S}^{\mathrm{cst}} \equiv \sin 2 \theta_{12}^{m} \sin \phi_{S},
$$

where

$$
\begin{aligned}
& \phi_{S}=\frac{\Delta m_{21}^{2} L}{4 E_{\nu}} \sqrt{\left(\cos 2 \theta_{12} \mp 2 V E_{\nu} / \Delta m_{21}^{2}\right)^{2}+\sin ^{2} 2 \theta_{12}}, \\
& \phi_{A}=\frac{\Delta m_{31}^{2} L}{4 E_{\nu}} \sqrt{\left(\cos 2 \theta_{13} \mp 2 V E_{\nu} / \Delta m_{31}^{2}\right)^{2}+\sin ^{2} 2 \theta_{13}} .
\end{aligned}
$$

Here the upper (lower) sign corresponds to neutrinos (antineutrinos).

Due to the level crossing phenomenon the factorization approximation (2.40) is not valid in the $\left(E_{\nu}, \Theta_{\nu}\right)$ parameter space of the 1-3 resonance where 1-3 mixing is enhanced. Indeed, using Eqs. (2.20) and (2.21) we find that for the normal mass hierarchy

$$
\phi_{32}^{m} \approx \phi_{A} \quad \text { for } \quad E_{\nu} \gg E_{12}^{R}
$$


and

$$
\phi_{21}^{m} \approx \phi_{S} \quad \text { for } \quad E_{\nu} \ll E_{13}^{R} .
$$

However, the last formula is not correct in the energy region near the 1-3 resonance and above it due to the 1-3 level crossing. In particular, from (2.21) we obtain for $E_{\nu} \gg E_{13}^{R}$

$$
\phi_{21}^{m} \approx \frac{L}{4 E_{\nu}}\left(\Delta m_{31}^{2} c_{13}^{2}-\Delta m_{21}^{2} c_{12}^{2}\right) \approx \frac{\Delta m_{31}^{2} L}{4 E_{\nu}} \equiv \phi_{A}^{0},
$$

where $\phi_{A}^{0}$ is the 1-3 vacuum phase. At the same time, $\phi_{S} \approx \phi_{31}^{m}$.

The results (2.26) and (2.27) allow us also to evaluate corrections to the factorization approximation. As follows from Eq. (2.26), beyond this approximation the amplitude $A_{e \tilde{2}}$ acquires an extra factor $c_{13}^{m}$ and still depends on just one oscillation frequency, determined by the solar mass splitting $\Delta m_{21}^{2}$. The factor $\cos \theta_{13}^{m}$ decreases with increasing neutrino energy, approaching the value $1 / \sqrt{2}$ at the $1-3 \mathrm{MSW}$ resonance and further decreasing to $\cos \theta_{13}^{m} \simeq 0$ above this resonance. This leads to an additional suppression of the "solar" amplitude $A_{e \tilde{2}}$ in matter at high energies, on top of the usual suppression due to the quenching of the mixing in the 1-2 sector.

According to Eq. (2.27) the exact expression for $A_{e \tilde{3}}^{\text {cst }}$ differs from $A_{A}^{\text {cst }}$ by an additional term depending on the 1-2 frequency. Above the 1-2 resonance we have $\cos ^{2} \theta_{12}^{m} \ll 1$, and this additional term can be omitted:

$$
A_{e \tilde{3}}^{\mathrm{cst}} \approx-i e^{i \phi_{21}^{m}}\left[\sin 2 \theta_{13}^{m} \sin \phi_{32}^{m} e^{-i \phi_{31}^{m}}\right] .
$$

Hence, up to a phase factor the amplitude $A_{e \tilde{3}}$ is also reduced to the standard two-neutrino form and depends on a single oscillation phase $\phi_{32}^{m}$. We can rewrite the amplitudes (2.26) and $(2.27)$ as

$$
\begin{array}{lr}
A_{e \tilde{2}}^{\mathrm{cst}}=-i e^{i \phi_{21}^{m}} \cos \theta_{13}^{m} A_{S}^{\mathrm{cst}} & \left(E_{\nu} \ll E_{13}^{R}\right), \\
A_{e \tilde{3}}^{\mathrm{cst}}=-i e^{i \phi_{21}^{m}}\left[e^{-i \phi_{31}^{m}} A_{A}^{\mathrm{cst}}+\cot ^{2} \theta_{12}^{m} \sin 2 \theta_{13}^{m} A_{S}^{\mathrm{cst}}\right], & \left(E_{12}^{R} \ll E_{\nu} \ll E_{13}^{R}\right) .
\end{array}
$$

In the general case of a matter of an arbitrary density profile, one can show, using simple power counting arguments, that the corrections to the factorization approximation for the amplitude $A_{e \tilde{2}}$ are of order $s_{13}^{2}$, whereas the corrections to the "atmospheric" amplitude $A_{e \tilde{3}}$ are of order $r_{\Delta}$ [50], in agreement with our consideration for constant density. The amplitude $A_{e \tilde{3}}$ does not in general have a 2 -flavor form, once the corrections to the factorization approximation are taken into account.

\section{Effects of 1-2 splitting and mixing}

\subsection{Neutrino oscillograms of the Earth}

In this section we study the neutrino oscillograms of the Earth - contours of constant oscillation probabilities in the plane of neutrino nadir angle and energy - in the complete $3 \nu$ context (see Fig. 1). In our numerical calculations we take the matter density distribution inside the Earth as given by the PREM model [57]. Unless otherwise specified, we assume 
the normal neutrino mass hierarchy and use the following numerical values of neutrino parameters: $\Delta m_{31}^{2}=2.5 \cdot 10^{-3} \mathrm{eV}^{2}, \Delta m_{21}^{2}=8 \cdot 10^{-5} \mathrm{eV}^{2}, \tan \theta_{12}=0.45[2,58,59,60]$.

Recall that the distance $L$ that neutrinos propagate inside the Earth is related to the nadir angle of the neutrino trajectory $\Theta_{\nu}$ through

$$
L=2 R_{\oplus} \cos \Theta_{\nu},
$$

where $R_{\oplus}=6371 \mathrm{~km}$ is the Earth radius. The value $\Theta_{\nu}=0$ corresponds to vertically upgoing neutrinos which travel along the Earth's diameter, whereas $\Theta_{\nu}=\pi / 2$ corresponds to horizontal neutrino trajectories. For $0 \leq \Theta_{\nu} \leq 33^{\circ}$ neutrinos cross both the mantle and the core of the Earth, whereas for larger values of the nadir angle they only cross the Earth's mantle. We call the corresponding parts of the oscillograms the core domain and the mantle domain, respectively.

In the limit of vanishing $\Delta m_{21}^{2}$ the main features of the neutrino oscillations in the Earth are determined by the MSW resonance enhancement of neutrino oscillations and the parametric resonance enhancement in the core domain (see Ref. [1] and Fig. 1). The former leads to the appearance of the MSW resonance peaks: one in the mantle domain of the oscillogram at neutrino energies $E_{\nu} \sim(6-7) \mathrm{GeV}$, and another one in the core domain at $E_{\nu} \sim(2-3) \mathrm{GeV}$. The latter (parametric enhancement) produces three parametric ridges in the core domain. In the notation of Ref. [1] they are ridges A, B, C. Ridge A spans the energy range from $E_{\nu} \approx 3 \mathrm{GeV}$ at $\Theta_{\nu}=0$ to $E_{\nu} \approx 6 \mathrm{GeV}$ at the mantle-core border, where it merges with the mantle MSW resonance peak (see Fig. 1 middle and right panels). Ridge B starts from $E_{\nu} \approx 5 \mathrm{GeV}$ at $\Theta_{\nu}=0$ and becomes nearly vertical for $\Theta_{\nu}=33^{\circ}$. The energy of the ridge $\mathrm{C}$ increases from $E_{\nu} \approx 10 \mathrm{GeV}$ to high energies when the nadir angle changes from $\Theta_{\nu}=0$ to $\Theta_{\nu}=33^{\circ}$. The location of the ridges weakly depends on the 1-3 mixing angle. The ridges differ by the oscillation phase acquired in the core [1].

\subsection{Oscillograms due to 1-2 mixing}

The oscillograms for $\theta_{13}=0$ are presented in the left panels of Fig. 1. In this limit, according to Eqs. (2.9) and (2.39),

$$
1-P_{e e}=\left|A_{e \tilde{2}}\right|^{2}=P_{S} .
$$

Thus, shown are the contours of constant probability $P_{S}$.

In the $2 \nu$ case the oscillation probabilities depend on $\Delta m^{2}$ and neutrino energy $E_{\nu}$ via the ratio $\Delta m^{2} / E_{\nu}$. Therefore when oscillations are driven by the solar splitting, $\Delta m_{21}^{2}$, the oscillation pattern is shifted to smaller energies as compared to that due to $\Delta m_{31}^{2}$. Moreover, the 1-2 pattern differs from the pattern for the 1-3 mixing due to the large value of the 1-2 mixing. Indeed, as a consequence of the large 1-2 mixing the following new features appear.

1. The oscillation length at the resonance is smaller than that for small mixing

$$
l_{m}^{R}=\frac{l_{\nu}}{\sin 2 \theta_{12}} \sim l_{\nu}
$$

where $l_{\nu}$ is the vacuum oscillation length. 


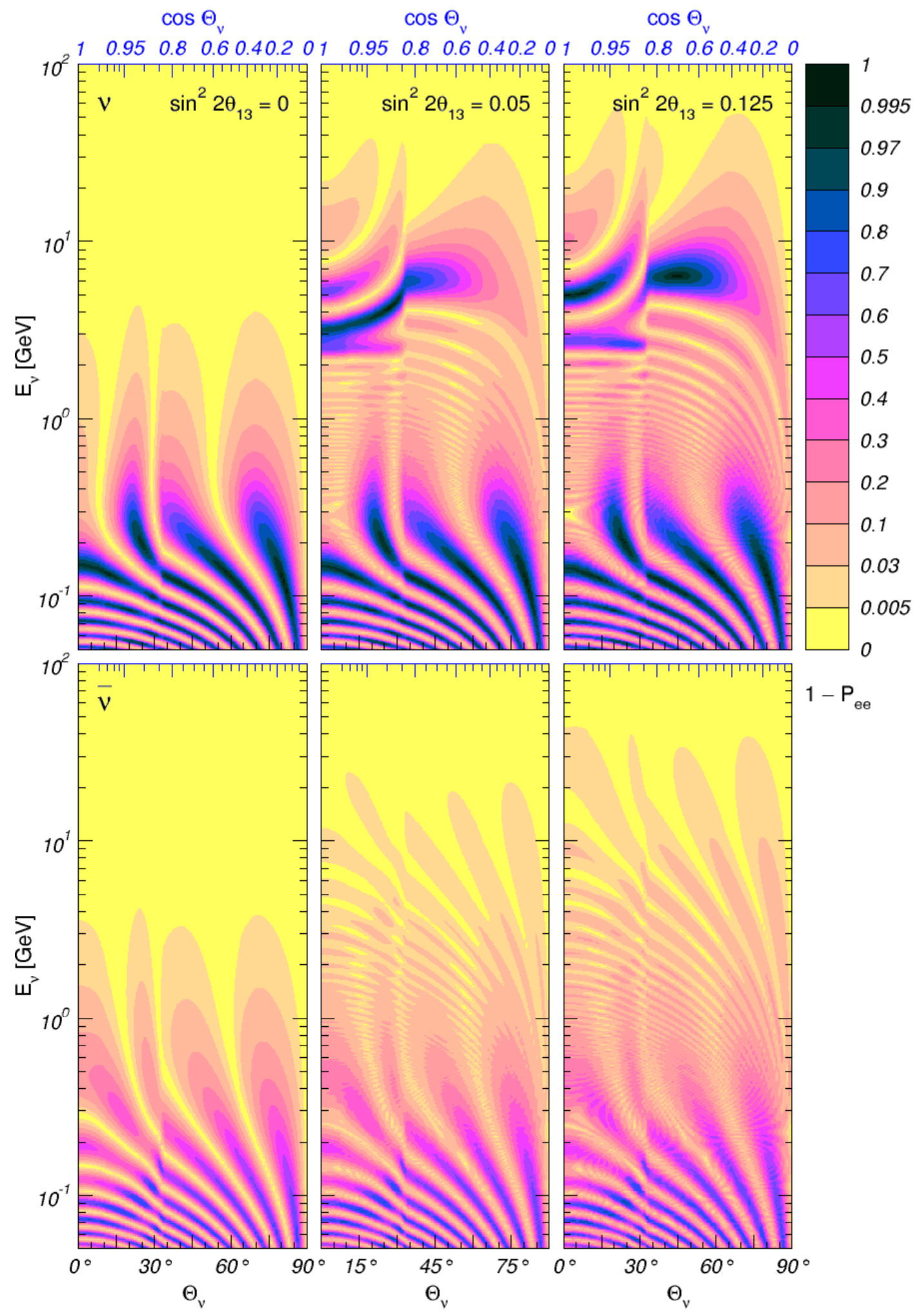

Figure 1: Neutrino oscillograms in the $3 \nu$-mixing case. Shown are the contours of constant probability $1-P_{e e}$ (upper panels) and $1-P_{\bar{e} \bar{e}}$ (lower panels) for $\Delta m_{21}^{2}=8 \times 10^{-5} \mathrm{eV}^{2}, \tan ^{2} \theta_{12}=0.45$ and different values of $\theta_{13}$. 
2. The resonance energy is shifted to smaller values not only due to $\Delta m_{21}^{2} \ll \Delta m_{31}^{2}$ but also because of the factor $\cos 2 \theta_{12} \approx 0.4$ :

$$
E_{12}^{R}=\frac{\Delta m_{21}^{2}}{2 \bar{V}} \cos 2 \theta_{12} .
$$

Here $\bar{V}$ is an effective (average) value of potential.

3. The degree of adiabaticity is better than for the 1-3 mixing case, and therefore the oscillation probability in the mantle is determined by the potential near the surface of the Earth, $\bar{V}$, averaged over the distance of the order of oscillation length.

4. The oscillation length in matter, $l_{m}=2 \pi / \Delta H_{21}$, monotonically increases with energy, approaching in the limit $E_{\nu} \rightarrow \infty$ the refraction length $l_{0} \equiv 2 \pi / V$. Recall that for small mixings $l_{m}$ first increases with energy, reaches a maximum slightly above the resonance and then decreases.

5. The jump of the mixing angle at the mantle-core boundary is small. Therefore, a sudden distortion of the oscillation patterns at $\Theta_{\nu}=33^{\circ}$ is not as significant as it is for the small 1-3 mixing, especially below the 1-2 resonance energy.

These features allow one to understand the structure of the oscillograms. In the mantle domain $\left(\Theta_{\nu}>33^{\circ}\right)$ the oscillation pattern for neutrinos is determined by the resonance enhancement of oscillations. There are three MSW resonance peaks above $0.1 \mathrm{GeV}$, which differ from each other by the value of the total oscillation phase. The outer peak $\left(\Theta_{\nu} \approx 82^{\circ}\right)$ corresponds to $\phi \approx \pi / 2$, the deeper one at $\Theta_{\nu}=60^{\circ}$, to $\phi \approx 3 \pi / 2$, and the inner one $\left(\Theta_{\nu} \approx 40^{\circ}\right)$, to $\phi=5 \pi / 2$. Recall that such a large phase can be acquired due to a smaller resonance oscillation length (3.3) in comparison to the length in the 1-3 mixing case, in which only one peak with $\phi=\pi / 2$ can be realized (see the upper parts of the panels in Fig. 1). The resonance energy is given by Eq. (3.4), and for the surface potential we find

$$
E_{12}^{R} \approx 0.12 \mathrm{GeV} .
$$

The ratio of the 1-2 and 1-3 resonance energies equals

$$
\frac{E_{12}^{R}}{E_{13}^{R}}=\left(\frac{\Delta m_{21}^{2}}{\Delta m_{31}^{2}}\right)\left(\frac{\cos 2 \theta_{12}}{\cos 2 \theta_{13}}\right)\left(\frac{\bar{V}_{13}}{\bar{V}_{12}}\right) \approx \frac{1}{50} .
$$

Here $\bar{V}_{13} / \bar{V}_{12} \approx 1.5$ (see [1]), since for 1-3 oscillations we should take the average of potential along the whole trajectory. The estimate (3.5) is valid for two outer peaks. For the peak at $\Theta_{\nu}=40^{\circ}, \bar{V}$ is larger, and accordingly, the resonance energy is slightly smaller.

The width of the 1-2 resonance is larger and therefore the regions of sizable oscillation probability are more extended in the $E_{\nu}$ direction as compared to the oscillations governed by the 1-3 mixing and splitting.

The resonance energy in the core is $E_{12}^{R} \approx 0.04 \mathrm{GeV}$. Therefore for $E_{\nu}>(0.10-$ $0.15) \mathrm{GeV}$ the 1-2 mixing in the core is substantially suppressed by matter. Furthermore, at the energies above the resonance energy in the mantle $\left(E_{\nu}>0.12 \mathrm{GeV}\right)$ the mixing in the mantle is also suppressed. Therefore the peaks with $P_{\max } \approx 1$ at $E_{\nu}>0.12 \mathrm{GeV}$ should be 

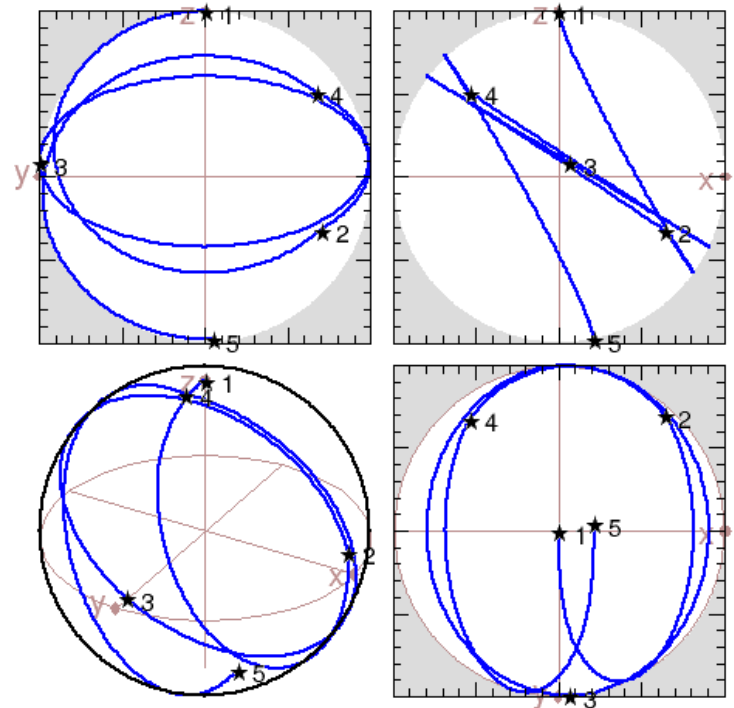

$\tan ^{2} \theta=0.45, \Theta_{v}=25.1^{\circ}, \mathrm{E}_{v}=0.2 \mathrm{GeV}$

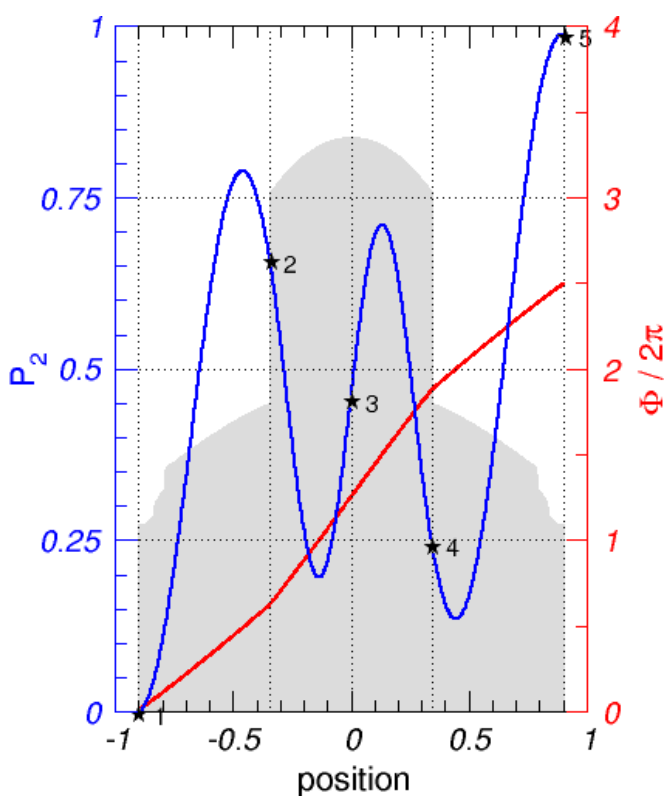

Figure 2: Parametric resonance due to 1-2 mixing and splitting at the parametric peak at $\Theta_{\nu}=$ $25.1^{\circ}$ and $E_{\nu}=0.2 \mathrm{GeV}$. Left panels: trajectory of the neutrino polarization vector in the flavor space, and projections of this trajectory onto three planes. Right panel: dependence of $P_{S}$ (blue line) and of the oscillation phase (red line) on the distance along the neutrino trajectory. The stars along the blue line correspond to the beginning (1), the mantle-core boundary (2), the midpoint (3), the core-mantle boundary (4), and the end of the trajectory (5).

due to the interplay of the core and mantle effects. In particular, the peak at $E_{\nu} \simeq 0.2 \mathrm{GeV}$ and $\Theta_{\nu} \simeq 25^{\circ}$ is due to the parametric enhancement of the oscillations. It corresponds to the realization of the parametric resonance condition when the oscillation half-phases equal approximately $\phi_{\text {mantle }} \approx \pi / 2$ and $\phi_{\text {core }} \approx 3 \pi / 2$ (note that the total phase $\approx 5 \pi / 2$, and this parametric ridge is attached to the $5 \pi / 2$ - MSW peak in the mantle domain). The spatial evolution of neutrinos in this peak and its graphical representation are shown in Fig. 2. In the left panel the blue lines present the trajectory of the neutrino polarization vector in the flavor space. Recall that in terms of the elements of the $2 \times 2$ evolution $S$ matrix, $S_{11}, S_{12}, S_{21}=-S_{12}^{*}$ and $S_{22}=S_{11}^{*}$ (where the last two equalities are the consequences of unitarity and hold in the basis where the effective Hamiltonian is traceless), the polarization vector is defined in the flavor space as the vector with components $s_{X}=\operatorname{Re}\left[S_{11}^{*} S_{12}\right]$, $s_{Y}=\operatorname{Im}\left[S_{11}^{*} S_{12}\right], s_{Z}=\left|S_{11}\right|^{2}-1 / 2$. Then the $\nu_{e}$ survival probability is given by $P_{e e}=$ $\left|S_{11}\right|^{2}=s_{Z}+1 / 2$ (see [1] for details).

The ridge at $E_{\nu} \simeq(0.12-0.15) \mathrm{GeV}$ and $\Theta_{\nu} \simeq 0-12^{\circ}$ can also be considered as being due to the parametric resonance with a larger core phase: $\phi_{\text {core }} \approx 5 \pi / 2$. However, for energies $E_{\nu} \leq 0.15 \mathrm{GeV}$ the in-matter mixing is nearly maximal both in the mantle and core, and the effect of the mantle-core density jump is small. As follows from Fig. 3 (left panel), the positions of the MSW peaks and maxima of the parametric ridges are determined very well by the intersections of the lines that correspond to the collinearity condition and the phase condition as in the case of oscillograms due to the 1-3 mixing [1]. 


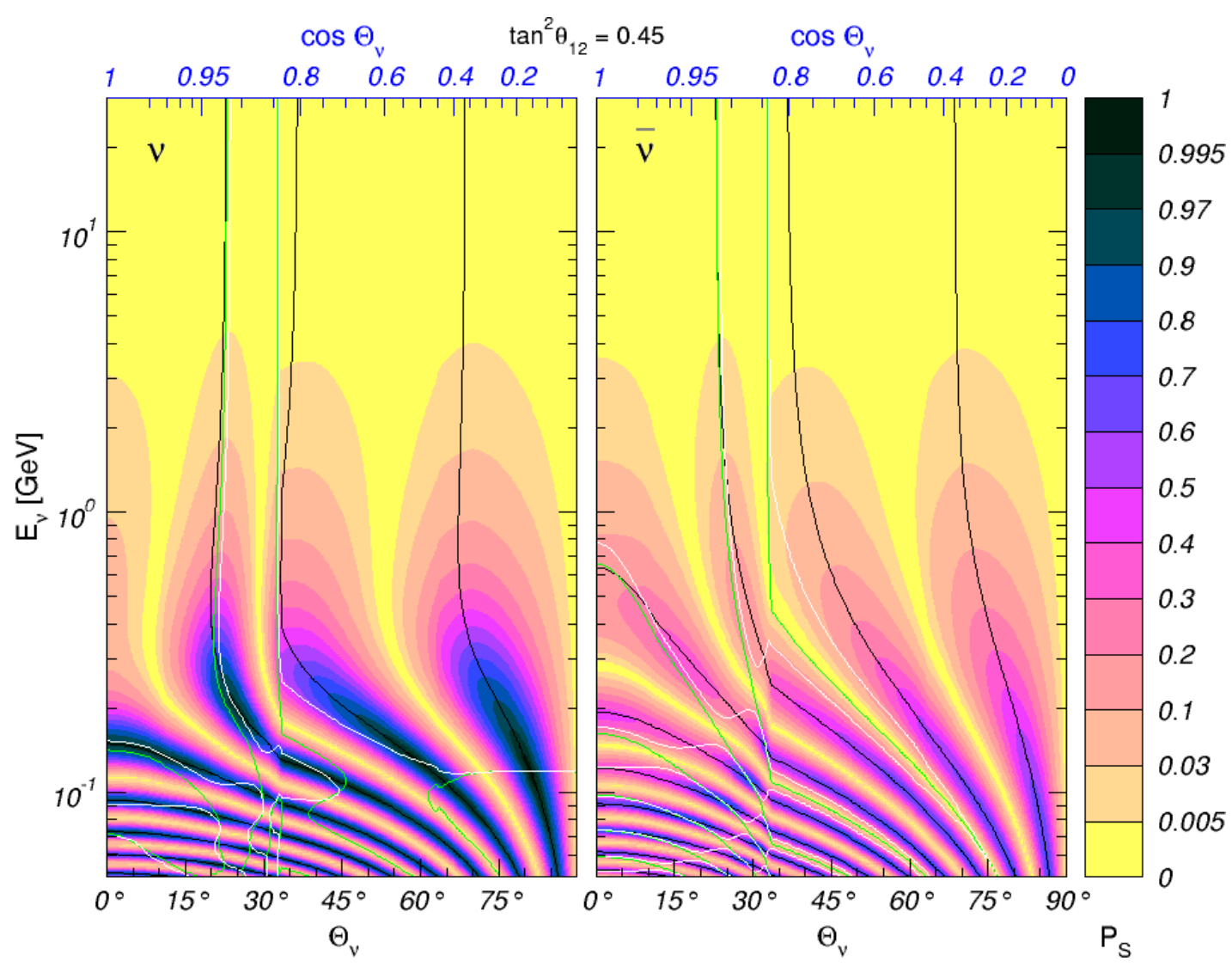

Figure 3: Interpretation of the oscillograms due to the 1-2 mixing and mass splitting, for neutrinos (left) and antineutrinos (right). Shown (in color) are contours of constant $P_{S}$ as well as lines of various conditions that explain different structures of the oscillograms. The lines correspond to collinearity condition (white), the generalized resonance condition (green), and the phase condition (black).

Recall that in terms of the elements of the evolution matrices in the mantle, $S^{m}$, and in the core, $S^{c}$, these conditions read

$$
\operatorname{Re}\left[S_{11}^{m} S_{11}^{c} S_{12}^{m}\right]=0 \text { (collinearity), } \quad \operatorname{Re}\left[\left(S^{m T} S^{c} S^{m}\right)_{11}\right]=0 \text { (phase). }
$$

In Fig. 3 shown are also the lines of the generalized resonance condition for symmetric density profile: $\operatorname{Im}\left[\left(S^{c} S^{m}\right)_{11}\right]=0$ (see [1] for details). The elements of the evolution matrix have been found by precise numerical computations. Apparently the ridges lie along the lines of the collinearity condition.

There are also several intersections of the collinearity and the phase condition lines in the core domain of the antineutrino oscillogram (right panel). This shows the existence of the parametric enhancement in the antineutrino channel.

For $E_{\nu}>0.3 \mathrm{GeV}$ the oscillation length practically does not depend on the neutrino energy and is close to the refraction length, $l_{0}=2 \pi /\left(\sqrt{2} G_{F} N_{e}\right)$. Therefore the lines of equal phases become nearly vertical. According to Fig. 3, the lines of zero oscillatory factor, $\phi=\pi k$ with $k=1,2,3$, that determine the so called "solar" magic lines (see [61]) 
are at $\Theta_{\nu} \sim 54^{\circ}$ in the mantle and at $\sim 30^{\circ}$ and $12^{\circ}$ in the core domain. The oscillation probabilities become smaller than $0.5 \%$ above $4 \mathrm{GeV}$ in the whole range of the nadir angles.

At lower energies, $E_{\nu}<0.1 \mathrm{GeV}$, one finds a regular oscillatory pattern with ridges and valleys. The distortion of this pattern at the core-mantle boundary is rather weak due to the smallness of the difference between the mixing angles in the mantle and core.

In the $2 \nu$ context the oscillation probabilities depend on $E_{\nu} / \Delta m^{2}$ and the mixing angle. Therefore with the increase of the mixing angle the oscillatory pattern obtained for the 1-3 mixing (upper parts of the oscillograms) should continuously transform into the pattern due to the 1-2 mixing (apart from the trivial shift of energy). We find that with increasing $\theta$ the parametric ridge A transforms first into the MSW peak in the mantle (1-3 mixing) and then to the outer MSW peak of the 1-2 pattern. The parametric ridge B transforms into the second MSW peak. The 1-3 core peak splits into two parts. One part transforms into the third MSW peak of the 1-2 pattern in the mantle. The second part merges with the parametric ridge $\mathrm{C}$ and appears as the parametric peak in the core domain at $E_{\nu}=0.2 \mathrm{GeV}$ at large mixings.

At high energies the patterns for neutrinos and antineutrinos are rather similar.

Turning on the non-zero 1-3 mixing leads to the appearance of an 1-3 oscillation pattern at high energies and to the interference of the 1-2 and 1-3 oscillation modes. We will discuss two types of the interference. The first one is the interference of modes characterized by the solar and atmospheric frequencies. The corresponding interference terms in probabilities do not necessarily depend on the $\mathrm{CP}$-violating phase. The second type yields the interference terms which depends on the CP-violating phase. We will call this the CP-interference.

In the following subsections we will consider the effects of the inclusion of the 1-2 mixing onto the 1-3 oscillatory pattern in different oscillation channels. We will compare the probabilities computed in the three-flavor $(3 \nu)$ context and in the two-flavor $(2 \nu)$ limit $\Delta m_{21}^{2} \rightarrow 0, \theta_{12} \rightarrow 0$. The $2 \nu$ probabilities are computed as $P\left(\Delta m_{31}^{2}, \sin ^{2} 2 \theta_{13}\right)$. That is, we take a single mass splitting in the $2 \nu$ context to coincide with the largest mass splitting in the $3 \nu$ case (the normal mass hierarchy). The oscillograms are computed for $\delta=0$.

\section{$3.3 \nu_{e}-\nu_{e}$ channel}

In Fig. 1 (upper panels) we show the probability $1-P_{e e}$ for three different values of $\sin ^{2} 2 \theta_{13}$ : zero (left), small (middle) and relatively large (right). As follows from the figure, in the first approximation the oscillograms for non-zero values of $\theta_{13}$ appear as superposition of the $2 \nu$ oscillation patterns produced by the 1-2 and 1-3 mixings with small interference effects. The smallness of the interference terms for this channel can be understood in the following way. According to Eq. (2.14), (2.15) and (2.18), the total probability of the $\nu_{e}$ disappearance is

$$
1-P_{e e}=P_{e \mu}+P_{e \tau}=P_{e \tilde{2}}+P_{e \tilde{3}}
$$

When the 1-2 splitting is neglected, this probability reduces to $P_{A}$, studied in detail in [1]. Eq. (3.8) shows that for $\Delta m_{21}^{2} \neq 0$ the $\nu_{e}-\nu_{\tilde{2}}$ and $\nu_{e}-\nu_{\tilde{3}}$ transition probabilities simply add up in $1-P_{e e}$ and no interference between the corresponding amplitudes (no CP-interference) 
occurs. Correspondingly, the probability $P_{e e}$ does not depend on the CP-violating phase in the standard parametrization. It does not depend on the 2-3 mixing either.

It follows from Eqs. (2.26) and (2.27) that $P_{e \tilde{2}}$ is driven only by the "solar" frequency $\left(\propto \Delta m_{21}^{2}\right)$, at least in the constant-density approximation, whereas $P_{e \tilde{3}}$ depends both on the "atmospheric" parameters $\left(\Delta m_{31}^{2}, \theta_{13}\right)$ and on the parameters of the 1-2 sector. Therefore the interference of the solar and atmospheric modes originates from $P_{e \tilde{3}} \equiv\left|A_{e \tilde{3}}\right|^{2}$. The interference of 1-2 and 1-3 oscillation modes can be quantified (at least for the mantle region) using the expression (2.27) valid for constant density matter:

$$
1-P_{e e}^{\mathrm{cst}}=\cos ^{2} \theta_{13}^{m}\left(1+\sin ^{2} \theta_{13}^{m} \cot ^{2} \theta_{12}^{m}\right) P_{S}^{\mathrm{cst}}+P_{A}^{\mathrm{cst}}+\cot \theta_{12}^{m} \sin 2 \theta_{13}^{m} \cos \phi_{31}^{m} A_{A}^{\mathrm{cst}} A_{S}^{\mathrm{cst}} .
$$

Here $A_{A}^{\text {cst }}$ and $A_{S}^{\text {cst }}$ are the $2 \nu$ amplitudes defined in (2.41) (note that in terms of $A_{A}^{\text {cst }}$ and $A_{S}^{\text {cst }}$ this expression is valid in the energy range between the two resonances. In the other energy ranges one needs to take into account the level crossing phenomenon, which changes the labeling of the phases). The last term in (3.9) is due to the interference of the solar and atmospheric modes which comes from $\left|A_{e \tilde{3}}\right|^{2}$. Apart from the product $A_{A}^{\text {cst }} A_{S}^{\text {cst }}$, this term contains additional small factors. In the region of the 1-2 resonance, $\theta_{13}^{m} \approx \theta_{13}, \cot \theta_{12}^{m} \sim 1$ and therefore the interference term is suppressed by a small factor $\sin 2 \theta_{13}$. In fact, all corrections to the main contribution, $P_{S}$, are of the order of the small probability $P_{A}^{\text {cst }} \sim \sin ^{2} \theta_{13}$. In the region of the $1-3$ resonance $P_{S}^{\text {cst }}$ is small, and the interference term is suppressed by $\cot \theta_{12}^{m} \sim r_{\Delta}$. Consequently, all the corrections to the dominant term $P_{A}^{\text {cst }}$ are of the order of $P_{S}^{\text {cst }}$. The interference term is further suppressed at the energies between the two resonances. Indeed, we can rewrite this term approximately as $\frac{1}{2} \sin 2 \theta_{12}^{m} \sin 2 \theta_{13}^{m} \cos \phi_{31}^{m} A_{A}^{\text {cst }} A_{S}^{\text {cst }}$. As we will see in Sec. 4.5 for small $\theta_{13}$ the product $\sin 2 \theta_{12}^{m} \sin 2 \theta_{13}^{m}$ has a minimum between the two resonances. Thus, the strong suppression of the effects of the 1-2 mixing in the $\nu_{e}-\nu_{e}$ channel is due to the absence of the CPinterference of the amplitudes $A_{e \tilde{2}}$ and $A_{e \tilde{3}}$.

To further illustrate the effects of the 1-2 mixing and mass splitting at high energies we show in Fig. 4 the oscillograms for $P_{e e}$ in the full three-flavor framework (left panels) and for the difference of probabilities with and without 1-2 mixing and splitting (right panels):

$$
\Delta P_{e e} \equiv P_{e e}-\stackrel{\circ}{P}_{e e}=P_{e e}-P_{e e}\left(\Delta m_{21}^{2}=0\right) .
$$

Recall that we compute the two-flavor probability $\stackrel{\circ}{P}_{e e}$ taking $\Delta m^{2}=\Delta m_{31}^{2}$.

In general, there are two contributions to $\Delta P_{e e}$ :

$$
\Delta P_{e e} \approx \Delta P_{e e}^{S}+\Delta P_{e e}^{A}
$$

which we will refer to as solar and atmospheric contributions. The solar contribution $\Delta P_{e e}^{S}$ is proportional to the solar amplitude, it includes term $P_{e e}^{S}$ and the interference of the amplitudes with the solar and atmospheric frequencies in $P_{e \tilde{3}}$. The second contribution in (3.11) follows from a change of the atmospheric mode: the phase and the amplitude of oscillations due to non-zero 1-2 mass splitting and mixing:

$$
\Delta P_{e e}^{A} \approx \Delta P^{A} \equiv P_{A}-\stackrel{\circ}{P}_{A} .
$$




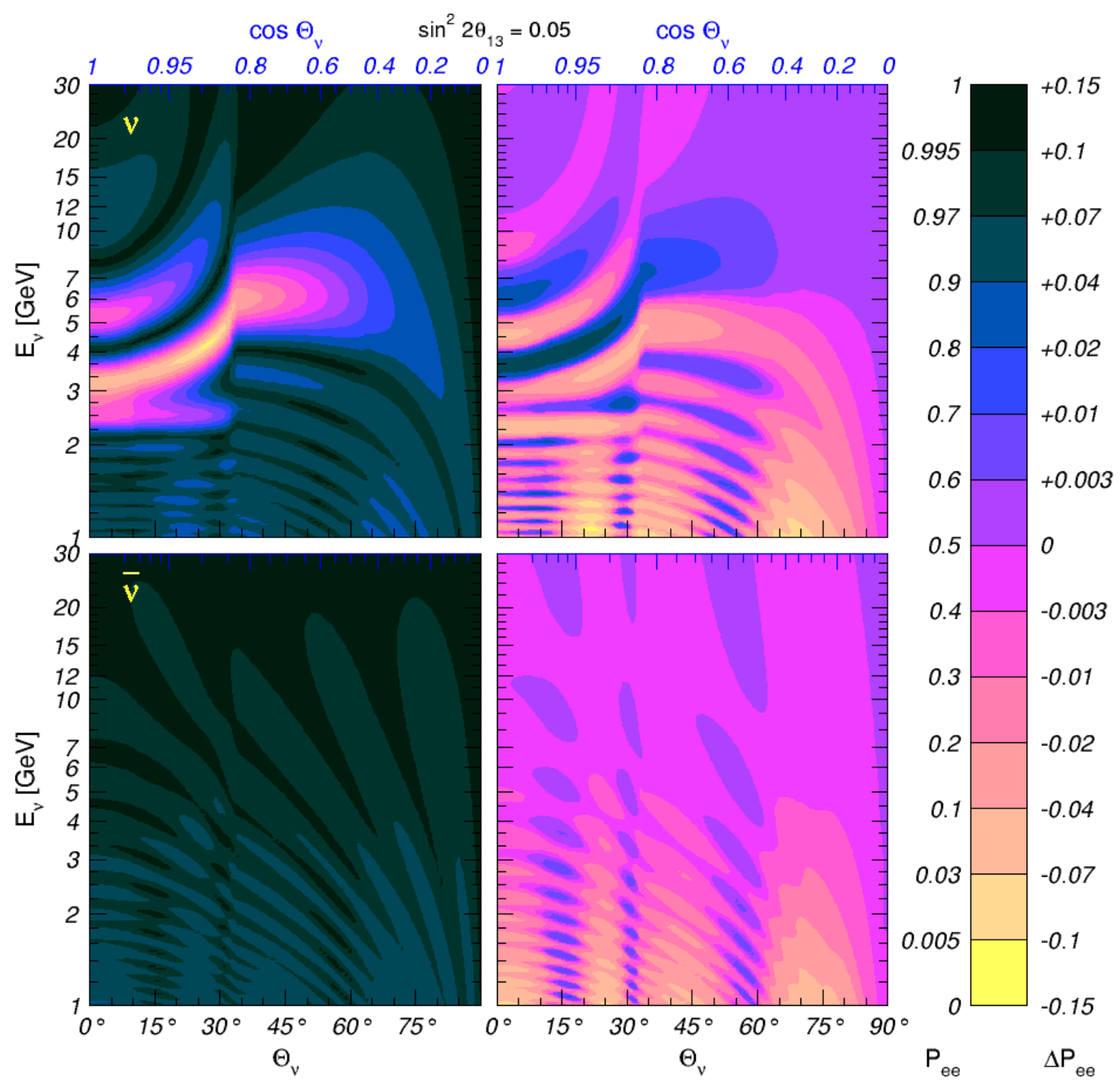

Figure 4: Oscillograms for the $\nu_{e}-\nu_{e}$ channel. Shown are the contours of constant probability $P_{e e}$ (left) as well as constant difference $\Delta P_{e e}$ of $3 \nu$ and $2 \nu$ probabilities (right), for neutrinos (upper panels) and antineutrinos (lower panels). The oscillation parameters for $3 \nu$ probabilities are $\sin ^{2} 2 \theta_{13}=0.05, \Delta m_{21}^{2}=8 \times 10^{-5} \mathrm{eV}^{2}, \tan ^{2} \theta_{12}=0.45$ and $\delta=0$. For the $2 \nu$ probabilities we used $\Delta m^{2}=\Delta m_{31}^{2}$.

Let us estimate these contributions using the results obtained for a matter of constant density. From Eq. (3.9) we have

$$
\Delta P_{e e}^{S} \approx \cos ^{2} \theta_{13}^{m} P_{S}^{\mathrm{cst}}+\cos \theta_{12}^{m} \sin 2 \theta_{13}^{m} \cos \phi_{31}^{m} A_{A}^{\mathrm{cst}} A_{S}^{\mathrm{cst}},
$$

and, according to our consideration above, for high energies $\Delta P_{e e}^{S}=\mathcal{O}\left(P_{S}^{\text {cst }}\right) \leq \sin ^{2} 2 \theta_{12}^{m}$, which is below 0.002 in the 1-3 resonance region. For the atmospheric contribution we obtain

$$
\Delta P^{A} \approx \sin ^{2} 2 \stackrel{\circ}{13}_{13}^{m} \sin ^{2} \stackrel{\circ}{\phi}_{31}^{m}-\sin ^{2} 2 \theta_{13}^{m} \sin ^{2} \phi_{32}^{m}
$$


Let us underline that due to the 1-2 level crossing, in the $3 \nu$ case the relevant atmospheric phase is $\phi_{32}^{m}$ and not $\phi_{31}^{m}$. In the lowest order

$$
\sin ^{2} 2 \theta_{13}^{m} \approx \sin ^{2} 2 \circ_{13}^{m}\left(V \rightarrow V_{1}\right)
$$

where

$$
V_{1} \approx \frac{V}{1-s_{12}^{2} r_{\Delta}}
$$

Then, using Eqs. (3.15) and (3.16), we obtain

$$
\Delta P^{A} \approx \sin ^{2} 2 \dot{\theta}_{13}^{m}\left[\Delta \phi \sin 2 \phi_{32}^{m}-2 \frac{\sin ^{2} 2 \theta_{13}^{m}}{\sin ^{2} 2 \theta_{13}} \sin ^{2} \phi_{32}^{m}\left(\cos 2 \theta_{13}-x\right) x s_{12}^{2} r_{\Delta}\right]
$$

where

$$
x \equiv \frac{2 V E_{\nu}}{\Delta m_{31}^{2}} .
$$

The first term in the brackets is proportional to the phase shift

$$
\Delta \phi=\stackrel{\circ}{\phi}_{31}^{m}-\phi_{32}^{m} \sim \frac{\Delta m_{21}^{2} L}{2 E_{\nu}},
$$

while the second one is due to the modification of the mixing angle. The doubly suppressed corrections, $\sim \Delta \phi r_{\Delta}$, are omitted. We find that for $\sin ^{2} 2 \theta_{13}=0.05$ both terms are of order $0.02-0.03$ in the region of the 1-3 resonance (in some regions of the nadir angles the second term can dominate).

The difference $\Delta P^{A}$ can be rewritten as

$$
\Delta P^{A} \approx 2 A_{A}^{\text {cst }} \sin 2 \theta_{13}^{m}\left[\cos \phi_{32}^{m} \Delta \phi-\frac{\sin ^{2} 2 \theta_{13}^{m}}{\sin ^{2} 2 \theta_{13}} \sin \phi_{32}^{m}\left(\cos 2 \theta_{13}-x\right) x s_{12}^{2} r_{\Delta}\right]
$$

so that its proportionality to $A_{A}^{\text {cst }}$ becomes manifest. Consequently, $\Delta P^{A}$ vanishes along the atmospheric magic lines, $A_{A}^{\text {cst }}=0$ [61] (see Sec. 4.2 for details).

Thus, for the $\nu_{e}-\nu_{e}$ channel, $\Delta P_{e e}^{A}$ dominates over $\Delta P_{e e}^{S}$ at high energies and therefore it describes the structure of the oscillograms for the probability differences. In particular, this explains the fact that oscillograms for $\Delta P_{e e}$ repeat the structure of $P_{e e}$ with certain shift in energy. Partly the difference $\Delta P_{e e}$ can be eliminated by modifying the $2 \nu$ value of $\Delta m_{31}^{2}$, taking $\Delta m^{2} \neq \Delta m_{31}^{2}$. In certain energy range the phase shift effect can be eliminated. Notice also that $\Delta P_{e e}^{A}$ is not proportional to $A^{S}$ or even to the corresponding oscillatory factor.

With the decrease of neutrino energy, the effect of the 1-2 mixing increases. Since $\Delta P^{A} \propto E_{\nu}^{-1}$ and $\Delta P^{S} \propto E_{\nu}^{-2}$, at lower energies the effect of the solar contribution becomes important. The interference effects of different modes are suppressed in $P_{e e}$, therefore the order of magnitude of the contributions to $1-P_{e e}$ due to nonzero 1-2 splitting can be readily estimated from the upper left panel of Fig. 1.

The oscillograms for the antineutrino channel $\bar{\nu}_{e} \rightarrow \bar{\nu}_{e}$ are shown in the lower panels of Figs. 1 and 4. Now apart from the change of the sign of $\delta$ one needs to take into account the change of the mixing and of the level crossing scheme. According to (2.23), the phases 
become $\phi_{A} \simeq \phi_{31}^{m}$ and $\phi_{S} \simeq \phi_{21}^{m}$. Therefore, the relevant oscillation phase in $A_{e \tilde{3}}^{\text {cst }}$ is $\phi_{31}^{m}$, and the amplitude (2.27) can be rewritten for antineutrinos as

$$
\bar{A}_{e \tilde{3}}^{\mathrm{cst}}=i e^{i \phi_{21}^{m}} \sin 2 \theta_{13}^{m}\left[\sin \phi_{31}^{m} e^{-i \phi_{32}^{m}}-\sin ^{2} \theta_{12}^{m} \sin \phi_{21}^{m}\right],
$$

where we used the phase exchange relation (2.37). At high energies $\sin ^{2} \theta_{12}^{m} \ll 1$, and the second term in (3.21) is very small. For the normal mass hierarchy both 1-2 and 1-3 mixings monotonically decrease with energy. The effect the of inclusion of the 1-2 mixing and splitting is illustrated in the lower panels of Fig. 4. Since for the antineutrino channel, in the absence of level crossing, the phase $\phi_{31}^{m}$ is relevant, the difference $\dot{\phi}_{31}^{m}-\phi_{31}^{m}$ is smaller than the corresponding difference in the neutrino channel. Thus, both $\Delta P_{A}$ and the total difference of the probabilities turn out to be smaller than they are in the neutrino channel. Furthermore, the solar contribution plays an important role now, determining the vertical structure of the oscillogram.

\section{$3.4 \nu_{e}-\nu_{\mu}$ and $\nu_{e}-\nu_{\tau}$ channels}

The transition probability $P_{\mu e} \equiv P\left(\nu_{\mu} \rightarrow \nu_{e}\right)$ (see (2.14)) can be rewritten as

$$
P_{\mu e}=c_{23}^{2}\left|A_{e \tilde{2}}\right|^{2}+s_{23}^{2}\left|A_{e \tilde{3}}\right|^{2}+\sin 2 \theta_{23}\left|A_{e \tilde{2}}^{*} A_{e \tilde{3}}\right| \cos (\phi-\delta),
$$

where

$$
\phi \equiv \arg \left(A_{e \tilde{2}}^{*} A_{e \tilde{3}}\right) .
$$

Unlike $1-P_{e e}$, this probability contains the interference term between $A_{e \tilde{2}}$ and $A_{e \tilde{3}}$ which depends on the CP-violation phase. Furthermore, this interference term is not suppressed by additional small factors as it happens in the $\nu_{e}-\nu_{e}$ channel. From the unitarity of the matrix $\tilde{S}$ in Eq. (2.6) we obtain for the product of amplitudes in the interference term

$$
A_{e \tilde{2}}^{*} A_{e \tilde{3}}=-A_{\tilde{3} \tilde{2}}^{*} A_{\tilde{3} \tilde{3}}-A_{\tilde{2} \tilde{3}} A_{\tilde{2} \tilde{2}}^{*},
$$

i.e., $A_{e \tilde{2}}^{*} A_{\tilde{3} e}$ is proportional to the small amplitudes $A_{\tilde{2} \tilde{3}}$ and $A_{\tilde{3} \tilde{2}}$.

Since the amplitude $A_{e \tilde{2}}$ is suppressed at high energies due to the smallness of the 1-2 mixing in matter, in the lowest approximation we have

$$
P_{\mu e} \approx \sin ^{2} \theta_{23}\left|A_{e \tilde{3}}\right|^{2} \approx \sin ^{2} \theta_{23}\left|A_{A}\right|^{2} .
$$

The maximal value of the probability $P_{\mu e} \simeq s_{23}^{2} \simeq 0.5$.

In the constant density approximation (valid for the mantle domain), using the amplitudes (2.27) we find at high energies

$$
P_{\mu e}^{\mathrm{cst}}=s_{23}^{2} P_{A}^{\mathrm{cst}}+\sin 2 \theta_{23} \cos \theta_{13}^{m} A_{A}^{\mathrm{cst}} A_{S}^{\mathrm{cst}} \cos \left(\phi_{31}^{m}+\delta\right)+\mathcal{O}\left(P_{S}^{\mathrm{cst}}\right) .
$$

Comparing this expression with (3.22) we find that for energies between the two resonances the interference phase satisfies

$$
\phi \approx-\phi_{31}^{m} .
$$

The precise expression for the phase in the constant density approximation can be obtained from $\phi \equiv \arg \left(A_{e \tilde{2}}^{\text {cst* }} A_{e \tilde{3}}^{\text {cst }}\right)=\arg \left(A_{e \tilde{3}}^{\text {cst }}\right)$. 


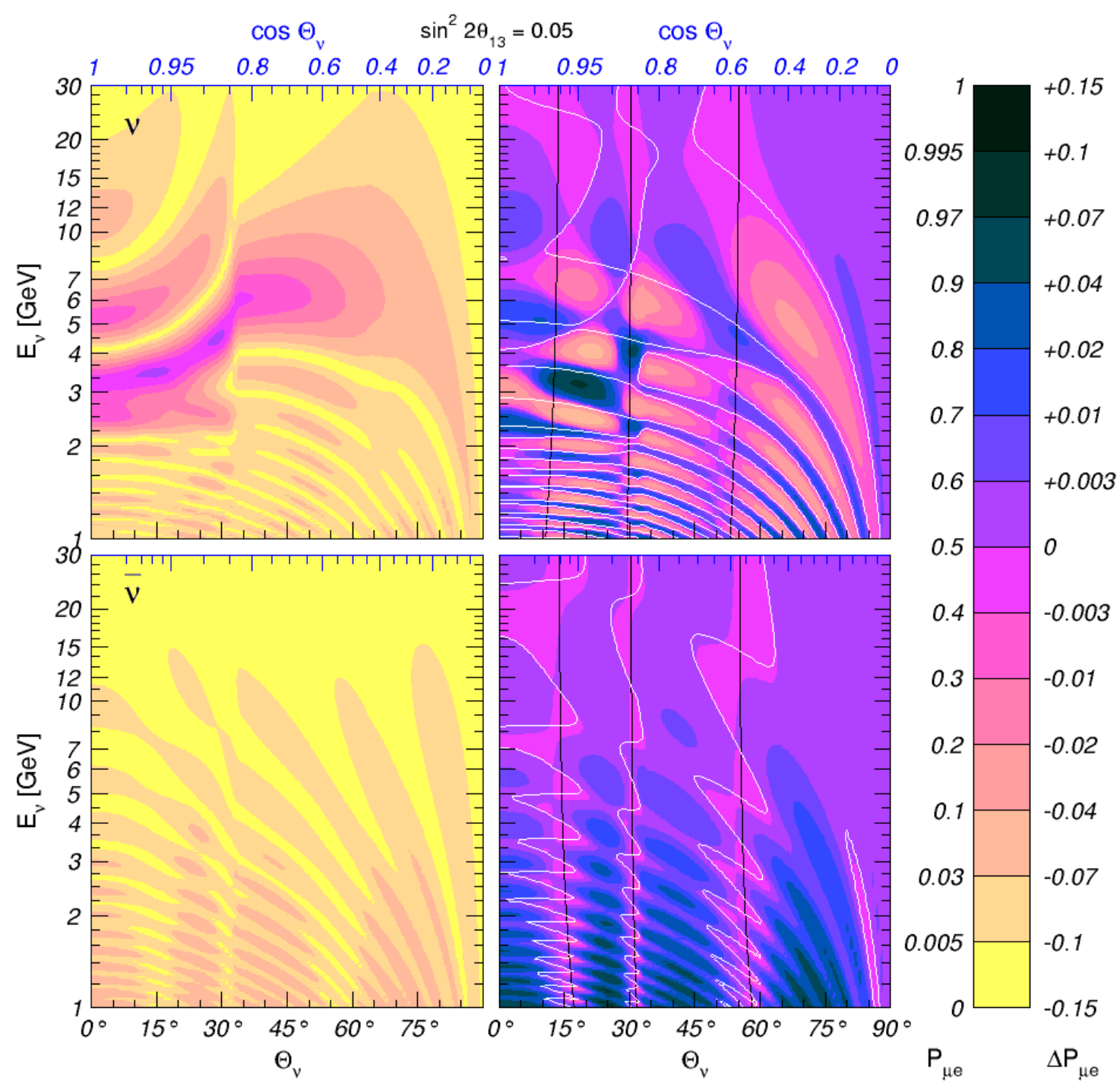

Figure 5: Oscillograms for the $\nu_{\mu}-\nu_{e}$ channel. Shown are the contours of constant probability $P_{\mu e}$ (left) as well as constant difference $\Delta P_{\mu e}$ of $3 \nu$ and $2 \nu$ probabilities (right), for neutrinos (upper panels) and antineutrinos (lower panels). The oscillation parameters for $3 \nu$ probabilities are $\sin ^{2} 2 \theta_{13}=0.05, \Delta m_{21}^{2}=8 \times 10^{-5} \mathrm{eV}^{2}, \tan ^{2} \theta_{12}=0.45$ and $\delta=0$. For the $2 \nu$ probabilities we used $\Delta m^{2}=\Delta m_{31}^{2}$. In the right panels we also show the solar magic lines (black) and the lines which correspond to the condition (3.32).

Fig. 5 illustrates the effect of inclusion of the 1-2 mixing and mass splitting on the $\nu_{\mu}-\nu_{e}$ and $\bar{\nu}_{\mu}-\bar{\nu}_{e}$ oscillation patterns for $E_{\nu} \geq 1 \mathrm{GeV}$. The left panels correspond to the $3 \nu$ case, whereas the right ones show the oscillograms for the difference of probabilities

$$
\Delta P_{\mu e} \equiv P_{\mu e}-P_{\mu e}\left(\Delta m_{21}^{2}=0\right)
$$

and, similarly, for $P_{\bar{\mu} \bar{e}}$ and $\Delta P_{\bar{\mu} \bar{e}}$. As in the $\nu_{e}-\nu_{e}$ case, this difference has two contributions:

$$
\Delta P_{\mu e} \approx \Delta P_{\mu e}^{S}+\Delta P_{\mu e}^{A}
$$


The solar amplitude contribution $\Delta P_{\mu e}^{S}$ is now dominated by the interference term and turns out to be much larger than in the $\nu_{e}-\nu_{e}$ case. The atmospheric contribution is determined by the same $\Delta P^{A}=P_{A}-\stackrel{\circ}{P}_{A}$ as before, Eqs. (3.17) and (3.20), with the additional factor $s_{23}^{2} \approx 0.5$ :

$$
\Delta P_{\mu e} \approx s_{23}^{2} \Delta P^{A}+\sin 2 \theta_{23} \cos \theta_{13}^{m} A_{A}^{\mathrm{cst}} A_{S}^{\mathrm{cst}} \cos \left(\phi_{31}^{m}+\delta\right) .
$$

Now in the 1-3 resonance region, the interference is $\leq 0.03-0.04$, whereas the atmospheric one $\leq 0.015$. Therefore, in the first approximation it is $\Delta P_{\mu e}^{S}$ that determines the structure of oscillograms for $\Delta P_{\mu e}$, whereas $\Delta P_{\mu e}^{A}$ leads to some corrections to this structure.

Using the factorization approximation we can write the solar contribution as

$$
\Delta P_{\mu e}^{S} \approx \cos \theta_{23}\left|A_{S}\right|\left(2 \sin \theta_{23}\left|A_{A}\right| \cos \left(\phi_{31}^{m}+\delta\right)+\cos \theta_{23}\left|A_{S}\right|\right) .
$$

This formula corresponds to the one in Eq. (3.22) and is valid for the energies below the energy of the 1-3 resonance. In the right panel of Fig. 5 we show the lines of vanishing solar correction: $\Delta P_{\mu e}^{S}=0$. This equality is satisfied where either $\left|A_{S}\right|=0$ (the solar magic lines), or

$$
2 \sin \theta_{23}\left|A_{A}\right| \cos \left(\phi_{31}^{m}+\delta\right)+\cos \theta_{23}\left|A_{S}\right|=0
$$

(white lines). If the second term is neglected the equality (3.32) splits into two conditions: $A_{A}=0$ (the atmospheric magic line, see Sec. 4.2) and $\cos \left(\phi_{31}^{m}+\delta\right)=0$ (the interference phase condition line). These lines form a grid which we will discuss in detail in Sec. 4. This grid describes rather well the domain structure of the oscillograms. Some deviations of the actual domain borders from the grid are related to the corrections from $\Delta P^{A}$. Representing $\Delta P^{A}$ in (3.17) as $\Delta P^{A}=A_{A}^{\text {cst }} K$, we can rewrite $\Delta P_{\mu e}$ in the constant density approximation in the following form (up to corrections $\mathcal{O}\left(P_{S}\right)$ ):

$$
\Delta P_{\mu e} \approx A_{A}^{\mathrm{cst}}\left[s_{23}^{2} K+\sin 2 \theta_{23} \cos \theta_{13}^{m} A_{S}^{\mathrm{cst}} \cos \left(\phi_{31}^{m}+\delta\right)\right] .
$$

Thus, with the atmospheric term taken into account, the lines $A_{A}^{\text {cst }}=0$ still determine zeros of the difference $\Delta P_{\mu e}$. However, the other lines of the condition $\Delta P_{\mu e}=0$ are shifted by the term $s_{23}^{2} K$ with respect to the solar magic and the interference phase lines.

Notice that the corrections to the $2 \nu$ oscillograms are enhanced in the 1-3 resonance region, especially in the core domain at $3-4 \mathrm{GeV}$, where $A_{A}$ is large due to the parametric resonance. Below $2 \mathrm{GeV}$ the corrections increase with decreasing energy because $\Delta P_{\mu e}^{S} \propto$ $P^{S} \propto 1 / E_{\nu}$ and $\Delta P_{\mu e}^{A} \propto 1 / E_{\nu}$.

One qualitatively new feature of the oscillograms with $\Delta m_{21}^{2} \neq 0$ is that the absolute minima of $P_{\mu e}$ appear there as isolated points (see Fig. 5). In contrast, in the limit $\Delta m_{21}^{2}=$ 0 the absolute minima of $P_{\mu e}$ (and $P_{\bar{\mu} \bar{e}}$ ) never appear as isolated points in the oscillograms, but always form continuous curves (valleys of zero probability). This is unlike for the absolute maxima, such as the MSW mantle peak or the parametric resonance peak in the core region, where even in the limit of zero 1-2 splitting the value $P_{A}=1$ (i.e., $\left.P_{\mu e}=s_{23}^{2} P_{A}=s_{23}^{2}\right)$ is reached only at a few isolated points. This feature is a consequence 
of the symmetry of the matter density profile of the Earth, and can be readily understood in the following way.

The condition for the absolute minimum of the transition probability, $P_{\mu e}=0$, or $S_{e \mu}=0$, where $S$ is the evolution matrix, can be written as

$$
\operatorname{Re}\left(S_{e \mu}\right)=0, \quad \operatorname{Im}\left(S_{e \mu}\right)=0,
$$

and for a generic profile the absolute minima of $P_{\mu e}$ are found as the points where the curves corresponding to the two conditions in (3.34) intersect. However, due to the symmetry of the Earth's matter density profile, in the 2-flavor approximation $\left(\Delta m_{21}^{2}=0\right)$ in the basis where the effective 2-flavor Hamiltonian of the system is traceless the transition amplitude is pure imaginary [11]. This means that the condition $\operatorname{Re}\left[S_{e \mu}\right]=0$ is satisfied automatically for all values of $E_{\nu}$ and $\Theta_{\nu}$. Therefore, the zeros of $P_{\mu e}$ simply coincide with the contour curves $\operatorname{Im}\left[S_{e \mu}\right]=0$. For $\Delta m_{21}^{2} \neq 0$ this is no longer the case, because the 2-flavor approximation does not apply.

For antineutrinos (lower panels of Fig. 5) the corrections are again determined by the interference term with somewhat smaller atmospheric contribution. Therefore, one can see a domain structure with vertical lines. With the decrease of energy the maxima of the corrections inside the domains monotonically increase, since so do both the solar and atmospheric amplitudes. Notice also that for $\delta=0$ the positive corrections are larger than negative; the situation in the neutrino channel is opposite.

As we pointed out in Sec. 2, for the inverse channel one has $P_{e \mu}=P_{\mu e}(\delta \rightarrow-\delta)$, where it has been taken into account that the Earth density profile is symmetric.

According to Eqs. (2.14) and (2.15) the oscillation probabilities $P_{\tau e}$ and $P_{e \tau}$ can be obtained from the corresponding probabilities $P_{\mu e}$ and $P_{e \mu}$ through the substitution $s_{23} \rightarrow$ $c_{23}, c_{23} \rightarrow-s_{23}$ [47]. The interference term has the opposite signs for channels including $\nu_{\tau}$ as compared with those with $\nu_{\mu}$, which can be obtained from the unitarity condition $P_{e e}+P_{\mu e}+P_{\tau e}=1$ and the fact that $P_{e e}$ does not depend on $\delta$.

\section{$3.5 \nu_{\mu}-\nu_{\mu}$ and $\nu_{\tau}-\nu_{\tau}$ channels}

The $\nu_{\mu}$ survival probability, $P_{\mu \mu}$, for symmetric density profiles is given in Eq. (2.16). It can be rewritten as

$$
\begin{aligned}
P_{\mu \mu}=\mid c_{23}^{2} A_{\tilde{2} \tilde{2}} & +\left.s_{23}^{2} A_{\tilde{3} \tilde{3}}\right|^{2} \\
& +2 \sin 2 \theta_{23} \cos \delta \operatorname{Re}\left[A_{\tilde{2} \tilde{3}}^{*}\left(c_{23}^{2} A_{\tilde{2} \tilde{2}}+s_{23}^{2} A_{\tilde{3} \tilde{3}}\right)\right]+\sin ^{2} 2 \theta_{23} \cos ^{2} \delta\left|A_{\tilde{2} \tilde{3}}\right|^{2} .
\end{aligned}
$$

Note that $P_{\mu \mu}$ is an even function of $\delta$. Since $A_{\tilde{2} \tilde{3}}=\mathcal{O}\left(r_{\Delta} s_{13}\right)$ is a small quantity, one can to a very good approximation neglect the term $\sim \cos ^{2} \delta$ in Eq. (2.16), which is proportional to $\left|A_{\tilde{2} \tilde{3}}\right|^{2}$. The term $\sim \cos ^{2} \delta$ can only become important when the main terms in $P_{\mu \mu}$ are small; however, this happens only in very small regions of the experimental parameter space.

In the limit $\Delta m_{21}^{2} \rightarrow 0$ we have $A_{\tilde{2} \tilde{2}}=1, A_{\tilde{2} \tilde{3}}=0$. Then, parametrizing the 33amplitude as

$$
A_{\tilde{3} \tilde{3}} \equiv\left|A_{\tilde{3} \tilde{3}}\right| e^{-i 2 \phi_{\tilde{3}}^{m}}=\sqrt{1-P_{A}} e^{-i 2 \phi_{\tilde{3}}^{m}}
$$


we obtain from (3.35)

$$
P_{\mu \mu}\left(\Delta m_{21}^{2}=0\right)=1-\sin ^{2} 2 \theta_{23} \sin ^{2} \phi_{\tilde{3} \tilde{3}}^{m}-s_{23}^{2} P_{A}-\sin ^{2} 2 \theta_{23} \cos 2 \phi_{\tilde{3} \tilde{3}}^{m}\left(1-\sqrt{1-P_{A}}\right) .
$$

If in addition $\theta_{13}=0$, then $P_{A}=0$ and $P_{\mu \mu}\left(\Delta m_{21}^{2}=0\right)$ is reduced to the standard $2 \nu$ vacuum oscillation probability with $\phi_{\tilde{3} \tilde{3}}^{m}=\phi_{A}^{0}$.

It is easy to estimate the effect of the 1-2 mixing in the limit $\theta_{13}=0$. In this case the eigenstate $\tilde{\nu}_{3}$ decouples in the propagation basis, $A_{\tilde{2} \tilde{3}}=0$, and the probability takes a very simple form

$$
P_{\mu \mu}=\left|c_{23}^{2} A_{\tilde{2} \tilde{2}}+s_{23}^{2} A_{\tilde{3} \tilde{3}}\right|^{2}
$$

Now

$$
A_{\tilde{3} \tilde{3}}=e^{-i 2 \phi_{\tilde{3} \tilde{3}}^{0}}, \quad \phi_{\tilde{3} \tilde{3}}^{0}=\frac{\Delta m_{31}^{2} L}{4 E_{\nu}},
$$

and parametrizing $A_{\tilde{2} \tilde{2}}$ as

$$
A_{\tilde{2} \tilde{2}}=\left|A_{\tilde{2} \tilde{2}}\right| e^{-i 2 \phi_{\tilde{2} \tilde{2}}^{m}}=\sqrt{1-P_{S}} e^{-i 2 \phi_{\tilde{2} \tilde{2}}^{m}},
$$

we obtain

$$
P_{\mu \mu}=1-\sin ^{2} 2 \theta_{23} \sin ^{2}\left(\phi_{\tilde{3} \tilde{3}}^{0}-\phi_{\tilde{2} \tilde{2}}^{m}\right)-c_{23}^{4} P_{S}-\frac{1}{2} \sin ^{2} 2 \theta_{23} \cos 2\left(\phi_{\tilde{3} \tilde{3}}^{0}-\phi_{\tilde{2} \tilde{2}}^{m}\right)\left(1-\sqrt{1-P_{S}}\right) .
$$

At high energies, $P_{S} \ll 1$, and consequently Eq. (3.41) becomes

$$
P_{\mu \mu}=P_{\mu \mu}^{2 \nu}-P_{S} c_{23}^{2}\left[c_{23}^{2}+s_{23}^{2} \cos 2\left(\phi_{\tilde{3} \tilde{3}}^{0}-\phi_{\tilde{2} \tilde{2}}^{m}\right)\right],
$$

where $P_{\mu \mu}^{2 \nu} \equiv 1-\sin ^{2} 2 \theta_{23} \sin ^{2}\left(\phi_{\tilde{3} \tilde{3}}^{m}-\phi_{\tilde{2} \tilde{2}}^{m}\right)$. In the constant density approximation and above the 1-2 resonance we have from $(2.32) \phi_{\tilde{2} \tilde{2}}^{m}=\phi_{21}^{m}$, and therefore $\phi_{\tilde{3} \tilde{3}}^{m}-\phi_{\tilde{2} \tilde{2}}^{m}=\phi_{32}^{m}$. Thus, the effect of the 1-2 mixing is reduced to a shift of the oscillation phase and small additive correction of order $P_{S}$. Larger corrections are expected for non-zero 1-3 mixing due to the interference of the 1-2 and 1-3 modes.

In Fig. 6 we show the oscillograms for $P_{\mu \mu}$ and $P_{\bar{\mu} \bar{\mu}}$ (left upper and lower panels) and the differences $\Delta P_{\mu \mu} \equiv P_{\mu \mu}-P_{\mu \mu}\left(\Delta m_{21}^{2}=0\right)$ and $\Delta P_{\bar{\mu} \bar{\mu}} \equiv P_{\bar{\mu} \bar{\mu}}-P_{\bar{\mu} \bar{\mu}}\left(\Delta m_{21}^{2}=0\right)$ (right panels). One can see in this figure the regular oscillatory pattern dominated by the vacuum $\nu_{\mu} \rightarrow \nu_{\tau}$ oscillations with certain distortion in the region of 1-3 the resonances: the MSW resonance in the mantle and core and the parametric ridges in the core domain. The dominant effect of the 1-2 mixing is related to the phase shift of this main mode, which increases with decreasing energy: at low energies the corrections become of order 1. $\Delta P_{\mu \mu}$ follows to a large extend the structure of $P_{\mu \mu}$. No domain structure appears here. All these features can be seen from the formulas (3.35), (3.37). In particular, according to (3.41), for zero 1-3 mixing we obtain

$$
\Delta P_{\mu \mu}=\sin ^{2} 2 \theta_{23}\left[\sin ^{2}\left(\phi_{\tilde{3} \tilde{3}}^{0}-\phi_{\tilde{2} \tilde{2}}^{m}\right)-\sin ^{2} \phi_{\tilde{3} \tilde{3}}^{0}\right]+\mathcal{O}\left(P_{S}\right) .
$$

An additional insight can be gained using the constant density approximation. Let us consider the case of maximal 2-3 mixing for which the figures have been plotted. For 


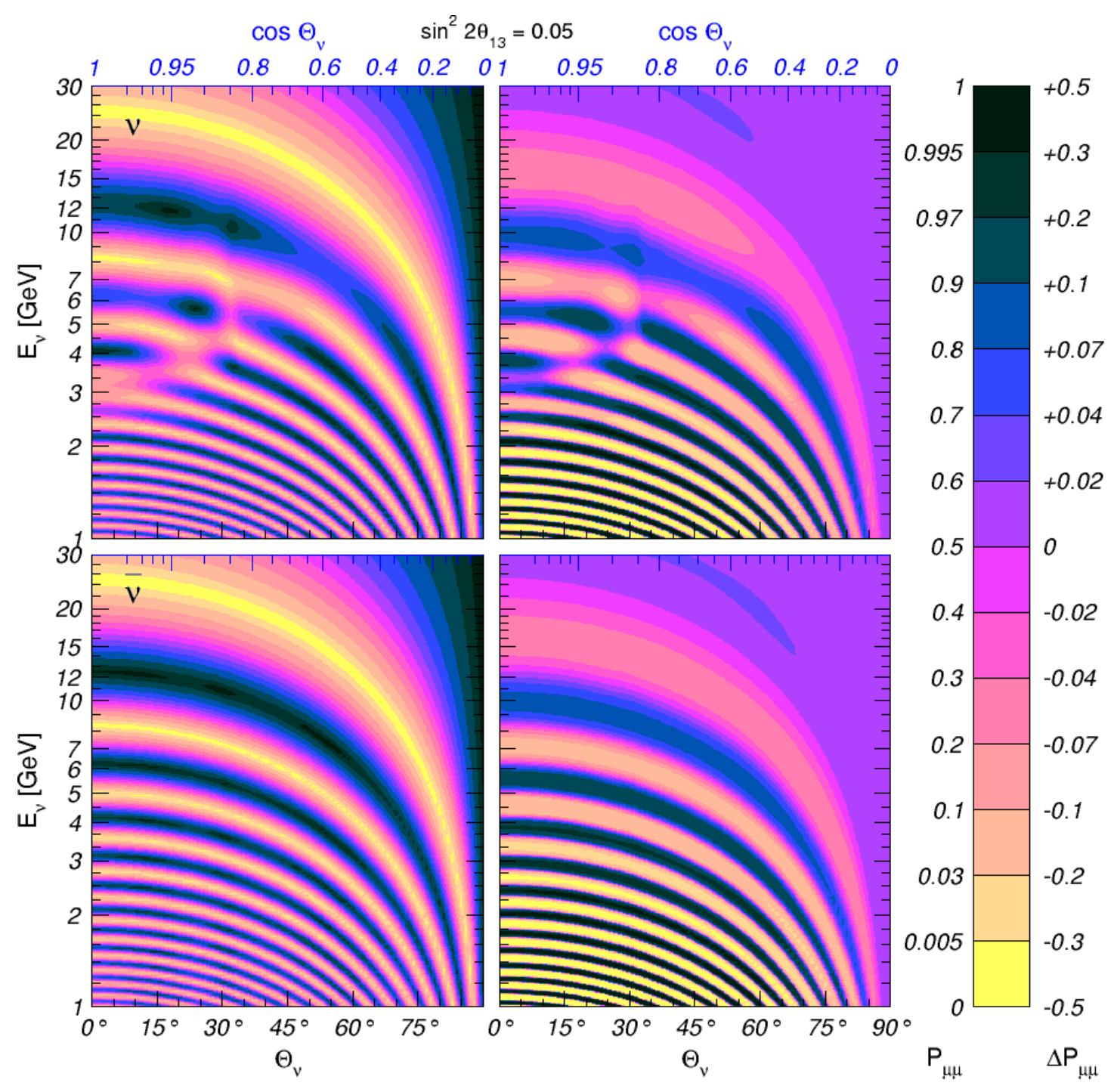

Figure 6: Oscillograms for the $\nu_{\mu}-\nu_{\mu}$ channel. Shown are the contours of constant probability $P_{\mu \mu}$ (left) as well as constant difference $\Delta P_{\mu \mu}$ of $3 \nu$ and $2 \nu$ probabilities (right), for neutrinos (upper panels) and antineutrinos (lower panels). The oscillation parameters for $3 \nu$ probabilities are $\sin ^{2} 2 \theta_{13}=0.05, \Delta m_{21}^{2}=8 \times 10^{-5} \mathrm{eV}^{2}, \tan ^{2} \theta_{12}=0.45$ and $\delta=0$. For the $2 \nu$ probabilities we used $\Delta m^{2}=\Delta m_{31}^{2}$.

maximal mixing and zero phase $\delta$ the interference term in the $\nu_{\mu}-\nu_{\mu}$ channel coincides, up to the sign, with the one in the $\nu_{\mu}-\nu_{e}$ channel (see Sec. 4), which has been estimated in the previous subsection. Therefore, let us now consider the first term in (3.35). In the limit $\Delta m_{21}^{2} \rightarrow 0$ we obtain for this term a very simple expression:

$$
P_{\mu \mu}\left(\Delta m_{21}^{2}=0\right)=1-\left(1-\sin ^{4} \theta_{13}^{m}\right) \sin ^{2} \stackrel{\circ}{\phi}_{32}^{m} .
$$

Here the phase $\stackrel{\circ}{\phi}_{32}^{m}$ should be calculated in the $2 \nu$ context with $H_{2}^{m}=0$.

The survival probabilities $P_{\tau \tau}$ and $P_{\bar{\tau} \bar{\tau}}$ can be obtained from the corresponding probabilities $P_{\mu \mu}$ and $P_{\bar{\mu} \bar{\mu}}$ through the substitution $s_{23} \rightarrow c_{23}, c_{23} \rightarrow-s_{23}$ [47]. 


\section{6 $\nu_{\mu}-\nu_{\tau}$ channel}

The probability of $\nu_{\mu} \rightarrow \nu_{\tau}$ oscillations for symmetric matter density profiles is given in (2.17). It can be rewritten as

$$
\begin{aligned}
P_{\mu \tau}=\frac{1}{4} \sin ^{2} 2 \theta_{23} \mid A_{\tilde{2} \tilde{2}} & -\left.A_{\tilde{3} \tilde{3}}\right|^{2}+\sin 2 \theta_{23} \cos 2 \theta_{23} \cos \delta \operatorname{Re}\left[\left(A_{\tilde{3} \tilde{3}}^{*}-A_{\tilde{2} \tilde{2}}^{*}\right) A_{\tilde{2} \tilde{3}}\right] \\
& -\sin 2 \theta_{23} \sin \delta \operatorname{Im}\left[A_{e \tilde{2}}^{*} A_{e \tilde{3}}\right]+\left(1-\sin ^{2} 2 \theta_{23} \cos ^{2} \delta\right)\left|A_{\tilde{2} \tilde{3}}\right|^{2} .
\end{aligned}
$$

The oscillations are mainly driven by $\Delta m_{31}^{2}$ and the large mixing angle $\theta_{23}$. The amplitude depends on $\delta$ through the terms proportional to $\cos \delta$ and $\sin \delta$, and therefore $P_{\mu \tau}$ contains both CP- and T-even and odd terms. Due to unitarity, all the information on $\nu_{\mu} \rightarrow \nu_{\tau}$ oscillations is contained in the already discussed probabilities $P_{\mu \mu}$ and $P_{\mu e}: P_{\mu \tau}=1-P_{\mu \mu}-$ $P_{\mu e}$. Furthermore, one can show that the $\delta$-dependent interference terms proportional to $\sin \delta$ and $\cos \delta$ satisfy the following relation

$$
P_{\mu \tau}^{\delta}=-P_{\mu e}^{\delta}-P_{\mu \mu}^{\delta}
$$

(see the next section for details).

Notice that for the maximal 2-3 mixing and $\delta=0$, the probability takes a very simple form

$$
P_{\mu \tau}=\frac{1}{4}\left|A_{\tilde{2} \tilde{2}}-A_{\tilde{3} \tilde{3}}\right|^{2} .
$$

In the limit $\Delta m_{21}^{2} \rightarrow 0$ we obtain

$$
P_{\mu \tau}\left(\Delta m_{21}^{2}=0\right)=\frac{1}{4}\left|1-A_{\tilde{3} \tilde{3}}\right|^{2}=\frac{1}{4}\left(2-P_{A}-2 \sqrt{1-P_{A}} \cos 2 \phi_{\tilde{3} \tilde{3}}^{m}\right),
$$

where $\phi_{\tilde{3} \tilde{3}}^{m}$ was defined in (3.36). For small $P_{A}$ the probability becomes

$$
P_{\mu \tau}\left(\Delta m_{21}^{2}=0\right)=\left(1-\frac{1}{2} P_{A}\right) \sin ^{2} \phi_{32}^{m}
$$

which in turn reduces to the standard $2 \nu$ probability for $\theta_{13}=0$.

The oscillograms for the $\nu_{\mu}-\nu_{\tau}$ channel are very similar to those for the $\nu_{\mu}-\nu_{\mu}$ channel plotted in Fig. 6. To a large extent they are complementary in the sense that the corresponding minima and maxima are interchanged. The $P_{\mu \tau}$ oscillograms exhibit the vacuum oscillations pattern everywhere apart from the region $E_{\nu} \simeq 3-12 \mathrm{GeV}$. In this region the pattern is distorted by the 1-3 level crossing, as well as by the parametric enhancement of the oscillations in the 1-3 mode. This distortion is absent in the antineutrino channel. As in the $\nu_{\mu}-\nu_{\mu}$ case, the difference of the probabilities, $\Delta P_{\mu \tau}$, is dominated by the phase shift, and the corrections have the opposite sign compared to $\Delta P_{\mu \mu}$.

Let us now present some results for constant density matter which will allow us to quantify the features described above. Using the expressions for the amplitudes in Eqs. (2.32) and (2.33), we find from Eq. (3.45) for $E_{\nu} \gg E_{12}^{R}$ (the maximal 2-3 mixing and $\delta=0$ )

$$
\begin{aligned}
P_{\mu \tau}^{\mathrm{cst}} & =\left|e^{-i \phi_{31}^{m}} \cos ^{2} \theta_{13}^{m} \sin \phi_{32}^{m}+\left[1-\cos ^{2} \theta_{12}^{m}\left(1+\sin ^{2} \theta_{13}^{m}\right)\right] \sin \phi_{21}^{m}\right|^{2} \\
& \approx\left|e^{-i \phi_{31}^{m}} \cos ^{2} \theta_{13}^{m} \sin \phi_{32}^{m}+\sin \phi_{21}^{m}\right|^{2} .
\end{aligned}
$$


For energies above the 1-3 resonance one, $\cos ^{2} \theta_{13}^{m} \rightarrow 0$ and Eq. (3.50) gives $P_{\mu \tau} \approx \sin ^{2} \phi_{21}^{m}$ with $\phi_{21}^{m} \approx \Delta m_{31}^{2} L / 2 E_{\nu}$. Therefore the corrections to $P_{\mu \tau}$ due to the vacuum 1-2 mixing and splitting are strongly suppressed. For energies between the two resonances, using the phase exchange relation (2.37), we obtain

$$
P_{\mu \tau}^{\mathrm{cst}} \approx \cos ^{4} \theta_{13}^{m} \sin ^{2} \phi_{31}^{m}+\frac{1}{2} \sin ^{2} 2 \theta_{13}^{m} \sin \phi_{21}^{m} \sin \phi_{31}^{m} \cos \phi_{32}^{m}+\sin ^{4} \theta_{13}^{m} \sin ^{2} \phi_{21}^{m} .
$$

For $\Delta m_{21}^{2}=0$ we have

$$
\begin{aligned}
P_{\mu \tau}^{\mathrm{cst}}\left(\Delta m_{21}^{2}=0\right) \approx \cos ^{4} \theta_{13}^{m} & \sin ^{2} \phi_{32}^{m} \\
& -\frac{1}{2} \sin ^{2} 2 \theta_{13}^{m} \sin \phi_{21}^{m} \sin \phi_{31}^{m} \cos \phi_{32}^{m}+\sin ^{4} \theta_{13}^{m} \sin ^{2} \phi_{21}^{m},
\end{aligned}
$$

where the mixing angle $\theta_{13}^{m}$ and the phases should be calculated in the $2 \nu$ context. From the level crossing scheme for the normal mass hierarchy we obtain for energies below the

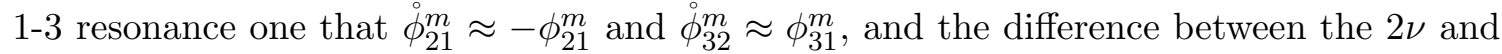
$3 \nu$ phases is proportional to $\Delta m_{21}^{2} L / 2 E_{\nu}$. This difference increases with decreasing energy and at $E_{\nu} \sim 1 \mathrm{GeV}$ it can be of order $\pi / 2$.

\subsection{Inverted mass hierarchy}

Let us briefly comment on the features of the oscillograms for the inverted mass hierarchy, i.e., for $\Delta m_{31}^{2}<0$. The main change as compared to the normal hierarchy is due to the 1-3 resonance structure which appears in the antineutrino channel now. Pulling out of the brackets in the Hamiltonian (2.5) the positive factor $\left|\Delta m_{31}^{2}\right|$, we have to change the sign in front of all the terms without $r_{\Delta}$ and $V$ in the matrix (2.5). The general oscillation formulas we presented before are valid in this case, however the eigenvalues of the Hamiltonian and mixing angles in matter should be changed. The level crossing scheme is also modified. In the neutrino channel (where there is the 1-2 resonance only), in the limit of large energies we have

$$
H_{1}^{m} \approx \frac{\Delta m_{21}^{2} c_{12}^{2}}{2 E_{\nu}}, \quad H_{2}^{m} \approx V, \quad H_{3}^{m} \approx \frac{\Delta m_{31}^{2}}{2 E_{\nu}} .
$$

In the antineutrino channel, below the 1-3 resonance the eigenvalues are

$$
H_{1}^{m} \approx V, \quad H_{2}^{m} \approx \frac{\Delta m_{21}^{2} c_{12}^{2}}{2 E_{\nu}}, \quad H_{3}^{m} \approx \frac{\Delta m_{31}^{2} c_{13}^{2}}{2 E_{\nu}},
$$

while above the 1-3 resonance,

$$
H_{1}^{m} \approx \frac{\Delta m_{31}^{2} c_{13}^{2}}{2 E_{\nu}}, \quad H_{2}^{m} \approx \frac{\Delta m_{21}^{2} c_{12}^{2}}{2 E_{\nu}}, \quad H_{3}^{m} \approx V .
$$

Here for illustration we will consider the oscillograms for the $\nu_{\mu}-\nu_{e}$ channel only. The other channels can be analyzed in a similar way. In Fig. 7 we show the oscillograms for $P_{\mu e}$ (left panels), and for the difference of $3 \nu$ and $2 \nu$ probabilities, $\Delta P_{\mu e}$ (right panels).

In the approximation of $\Delta m_{21}^{2}=0$ the neutrino oscillograms for the inverted hierarchy coincide with the antineutrino oscillograms for the normal hierarchy, and vice-versa, provided that $\Delta m_{31}^{2}$ are taken to be the same in both cases. The inclusion of the 1-2 mixing 


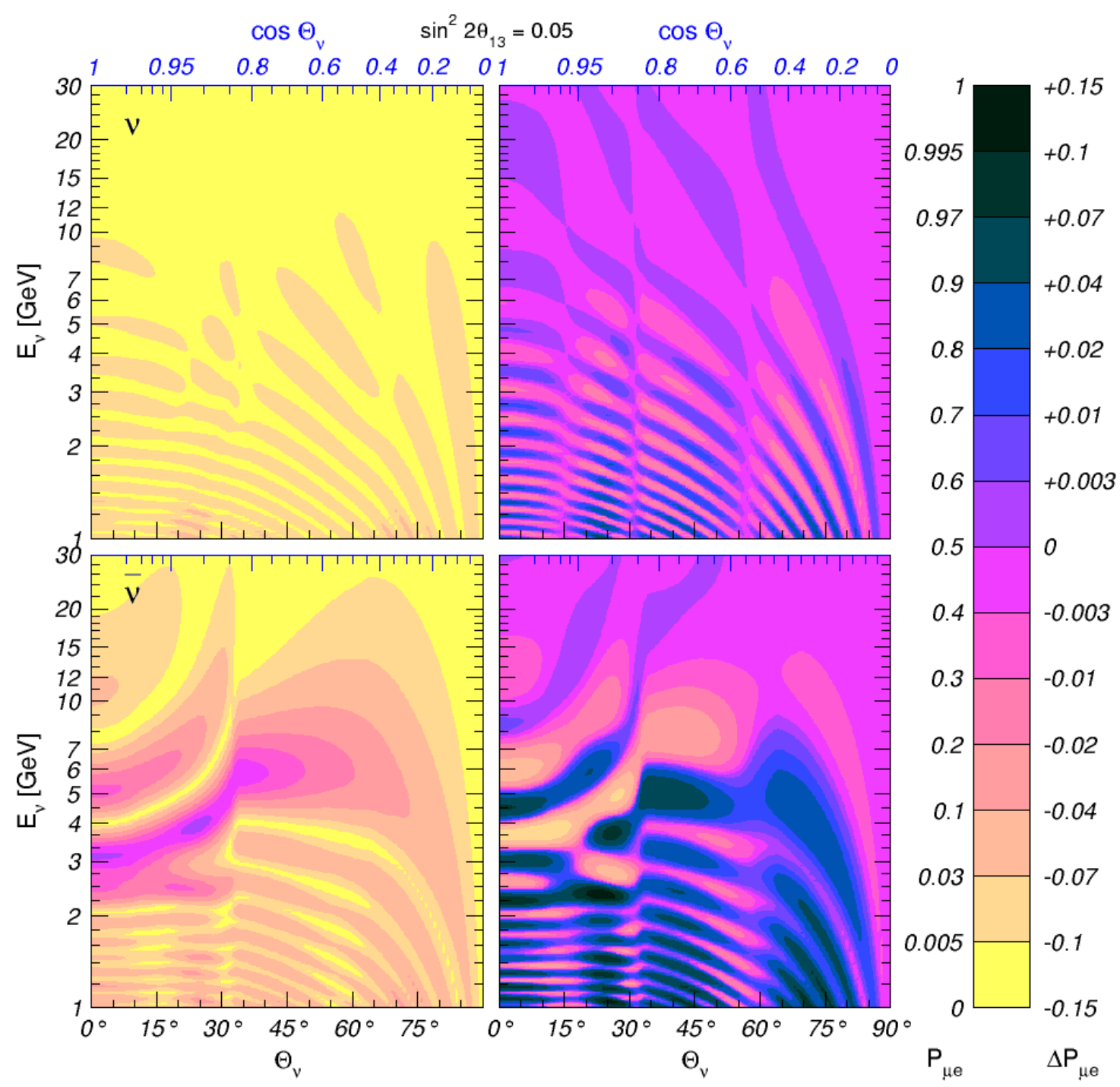

Figure 7: Oscillograms for the $\nu_{\mu}-\nu_{e}$ channel in the case of the inverted mass hierarchy. Shown are the contours of constant probability $P_{\mu e}$ (left) as well as constant difference $\Delta P_{\mu e}$ of $3 \nu$ and $2 \nu$ probabilities (right), for neutrinos (upper panels) and antineutrinos (lower panels). The oscillation parameters for $3 \nu$ probabilities are $\sin ^{2} 2 \theta_{13}=0.05, \Delta m_{21}^{2}=8 \times 10^{-5} \mathrm{eV}^{2}, \tan ^{2} \theta_{12}=0.45$ and $\delta=0$. For the $2 \nu$ probabilities we used $\Delta m^{2}=\Delta m_{31}^{2}$.

and mass splitting breaks this symmetry. However, at high energies where the corrections are small, the correspondence " $\nu$ inverted $\leftrightarrow \bar{\nu}$ normal", " $\bar{\nu}$ inverted $\leftrightarrow \nu$ normal" approximately holds. In our computations in the limit $\Delta m_{21}^{2} \rightarrow 0$ we have taken the remaining mass squared difference to be equal to the largest splitting in the $3 \nu$ context, $\Delta m^{2}=\Delta m_{32}^{2}$.

In the neutrino channel now there is only the 1-2 resonance and the height of the oscillation peaks of the probability increases with decreasing energy. The difference of $3 \nu$ and $2 \nu$ probabilities is mainly due to the interference terms, as in the normal mass hierarchy case. This explains why one can clearly see a domain structure with vertical lines. The solar magic lines are shifted due to the contribution from $\Delta P^{A}$. At high energies negative 
corrections are larger in the absolute value than the positive ones (for $\delta=0$ ). Notice also that the shape of domains for neutrinos in the case of inverted hierarchy is similar to that for antineutrinos in the normal mass hierarchy case.

In the antineutrino channel the oscillation pattern is dominated by the MSW and parametric resonances. The difference of probabilities in the $3 \nu$ and $2 \nu$ contexts is again due to the interference term $\propto A_{S} A_{A}$, with some corrections from $\Delta P_{A}$. According to the figure, the 1-2 mixing and splitting effect is enhanced in the regions of the MSW resonance peaks and along the parametric ridges, since $A_{A}$ is enhanced there. The oscillogram $\Delta P_{\mu e}$ has a domain structure. The heights of the peaks are maximal in the resonance regions. In the mantle domain the heights reach minimum at $E_{\nu} \sim 3 \mathrm{GeV}$ and in the core domain at $E_{\nu} \sim 2 \mathrm{GeV}$, similarly to what we had for the CP-sensitivity peaks (Sec. 4). Due to the interplay of the interference terms and $\Delta P^{A}$, for $\delta=0$ the corrections in the case of the inverted mass hierarchy are larger than those in the normal mass hierarchy case: e.g., in the region of the 1-3 resonance we have $\Delta P_{\mu e} \sim \pm(0.07-0.10)$. Below $3-4 \mathrm{GeV}$ the positive corrections dominate.

One can use the analytic formulas of Sec. 3 with appropriately changed phases and mixing angles to describe these results quantitatively.

\section{Effects of CP-violating phase $\delta$}

In this section we consider in detail the properties of the CP-interference terms and in particular their dependence on the phase $\delta$ in different channels. As can be seen from the expressions given in Sec. 2, the survival probability $P_{e e}$ does not depend on the CP-violating phase $\delta$, both for oscillations in vacuum and in matter $[34,38]$. This is a consequence of the facts that (i) $\delta$ is rotated away by transforming to the propagation basis, and (ii) the probability $P_{e e}$ is not affected by this transformation. Note that for oscillations in vacuum or in matter with symmetric density profiles, the other two survival probabilities, $P_{\mu \mu}$ and $P_{\tau \tau}$, depend on $\delta$ only through the terms proportional to $\cos \delta$ and $\cos 2 \delta$ [43] since they are T-even quantities. In contrast to this, for oscillations in a matter whose density profile is not symmetric with respect to the midpoint of the neutrino trajectory, these probabilities acquire also terms proportional to $\sin \delta$ and $\sin 2 \delta$.

\subsection{Interference and CP-violation}

The unitarity of the evolution matrix in the propagation basis (2.6) for symmetric density profiles gives

$$
A_{\tilde{2} \tilde{3}} A_{\tilde{2} \tilde{2}}^{*}+A_{\tilde{2} \tilde{3}}^{*} A_{\tilde{3} \tilde{3}}=-A_{e \tilde{2}}^{*} A_{e \tilde{3}} \cdot
$$

This relation allows one to write explicitly the $\delta$-dependent terms for different oscillation channels as

$$
\begin{aligned}
& P_{\mu e}^{\delta}=\sin 2 \theta_{23}\left\{\cos \delta \operatorname{Re}\left[A_{e \tilde{2}}^{*} A_{e \tilde{3}}\right]+\sin \delta \operatorname{Im}\left[A_{e \tilde{2}}^{*} A_{e \tilde{3}}\right]\right\} \\
& P_{\mu \mu}^{\delta}=\sin 2 \theta_{23} \cos \delta\left\{-\operatorname{Re}\left[A_{e \tilde{2}}^{*} A_{e \tilde{3}}\right]-\cos 2 \theta_{23} \operatorname{Re}\left[A_{\tilde{2} \tilde{3}}^{*}\left(A_{\tilde{3} \tilde{3}}-A_{\tilde{2} \tilde{2}}\right)\right]\right\} \\
& P_{\mu \tau}^{\delta}=\sin 2 \theta_{23}\left\{-\sin \delta \operatorname{Im}\left[A_{e \tilde{2}}^{*} A_{e \tilde{3}}\right]+\cos \delta \cos 2 \theta_{23} \operatorname{Re}\left[A_{\tilde{2} \tilde{3}}^{*}\left(A_{\tilde{3} \tilde{3}}-A_{\tilde{2} \tilde{2}}\right)\right]\right\}
\end{aligned}
$$


Here in $P_{\mu \mu}^{\delta}$ and $P_{\mu \tau}^{\delta}$ we have omitted small terms proportional to $\left|A_{\tilde{2} \tilde{3}}\right|^{2}$. The sum of these interference terms is zero. For maximal 2-3 mixing Eqs. (4.2), (4.3) and (4.4) reduce to

$$
\begin{aligned}
P_{\mu e}^{\delta} & =\cos \delta \operatorname{Re}\left[A_{e \tilde{2}}^{*} A_{e \tilde{3}}\right]+\sin \delta \operatorname{Im}\left[A_{e \tilde{2}}^{*} A_{e \tilde{3}}\right] \\
P_{\mu \mu}^{\delta} & =-\cos \delta \operatorname{Re}\left[A_{e \tilde{2}}^{*} A_{e \tilde{3}}\right] \\
P_{\mu \tau}^{\delta} & =-\sin \delta \operatorname{Im}\left[A_{e \tilde{2}}^{*} A_{e \tilde{3}}\right] .
\end{aligned}
$$

The following consequences of these expressions can be useful for measurements of $\delta$ : if $\delta=0$, the probability $P_{\mu \tau}$ does not contain the interference term and $P_{\mu \mu}^{\delta}=-P_{\mu e}^{\delta}$; if $\delta=\pi / 2, P_{\mu \mu}$ has no interference term and $P_{\mu \tau}^{\delta}=-P_{\mu e}^{\delta}$.

Using the phase $\phi \equiv \arg \left(A_{e \tilde{2}}^{*} A_{e \tilde{3}}\right)$ defined in Eq. (3.23) we obtain in the general case

$$
\begin{aligned}
& P_{\mu e}^{\delta}=\sin 2 \theta_{23} \cos (\phi-\delta)\left|A_{e \tilde{2}} A_{e \tilde{3}}\right|, \\
& P_{\mu \mu}^{\delta}=-\sin 2 \theta_{23} \cos \delta \cos \phi\left|A_{e \tilde{2}} A_{e \tilde{3}}\right|-D_{23}, \\
& P_{\mu \tau}^{\delta}=-\sin 2 \theta_{23} \sin \delta \sin \phi\left|A_{e \tilde{2}} A_{e \tilde{3}}\right|+D_{23},
\end{aligned}
$$

where

$$
D_{23} \equiv \frac{1}{2} \sin 4 \theta_{23} \cos \delta \operatorname{Re}\left[A_{\tilde{2} \tilde{3}}^{*}\left(A_{\tilde{3} \tilde{3}}-A_{\tilde{2} \tilde{2}}\right)\right]
$$

is proportional to the deviation of the 2-3 mixing from the maximal one. Notice that $D_{23}$ enters into $P_{\mu \mu}^{\delta}$ and $P_{\mu \tau}^{\delta}$ with opposite signs while $P_{\mu e}^{\delta}$ does not depend on $D_{23}$ at all. $D_{23}$ is CP-even. It can be estimated using the constant density approximation as

$$
D_{23}^{\mathrm{cst}} \approx-\frac{1}{2} \sin 4 \theta_{23} \cos \delta\left[\cos \phi_{31}^{m} c_{13}^{m} A_{A}^{\mathrm{cst}} A_{S}^{\mathrm{cst}}-2 \sin \theta_{13}^{m} \sin \phi_{21}^{m} A_{S}^{\mathrm{cst}}\right] .
$$

This expression shows that corrections to the term proportional to $A_{A}^{\text {cst }} A_{S}^{\text {cst }}$ in $D_{23}$ are in general not small.

For maximal 2-3 mixing one has

$$
\begin{aligned}
& P_{\mu e}^{\delta}=\cos (\phi-\delta)\left|A_{e \tilde{2}} A_{e \tilde{3}}\right|, \\
& P_{\mu \mu}^{\delta}=-\cos \delta \cos \phi\left|A_{e \tilde{2}} A_{e \tilde{3}}\right| \\
& P_{\mu \tau}^{\delta}=-\sin \delta \sin \phi\left|A_{e \tilde{2}} A_{e \tilde{3}}\right|
\end{aligned}
$$

The other $\delta$-dependent terms in the probabilities, which are proportional to the square of the small quantity $\left|A_{\tilde{2} \tilde{3}}\right|^{2}$, are

$$
P_{\mu \mu}^{\delta \delta} \equiv \cos ^{2} \delta \sin ^{2} 2 \theta_{23}\left|A_{\tilde{2} \tilde{3}}\right|^{2}, \quad P_{\mu \tau}^{\delta \delta} \equiv\left(1-\cos ^{2} \delta \sin ^{2} 2 \theta_{23}\right)\left|A_{\tilde{2} \tilde{3}}\right|^{2} .
$$

Notice that the sum of these terms does not depend on $\delta$ and equals $\left|A_{\tilde{2} \tilde{3}}\right|^{2}$.

Let us present also the $\delta$ - dependent terms of the probabilities for other channels. As we have already mentioned, $P_{\tau e}^{\delta}=-P_{\mu e}^{\delta}$, and for the reverse channels, according to (2.18) we obtain

$$
\begin{aligned}
& P_{e \mu}^{\delta}=\sin 2 \theta_{23} \cos (\phi+\delta)\left|A_{e \tilde{2}} A_{e \tilde{3}}\right| \\
& P_{\tau \mu}^{\delta}=\sin 2 \theta_{23} \sin \delta \sin \phi\left|A_{e \tilde{2}} A_{e \tilde{3}}\right|+D_{23}
\end{aligned}
$$


As was pointed out above, $P_{\tau \tau}$ can be obtained from $P_{\mu \mu}$ through the substitution $s_{23} \rightarrow$ $c_{23}, c_{23} \rightarrow-s_{23}:$

$$
P_{\tau \tau}^{\delta}=\sin 2 \theta_{23} \cos \delta \cos \phi\left|A_{e \tilde{2}} A_{e \tilde{3}}\right|-D_{23} .
$$

For antineutrinos, according to (2.19), the probabilities have the same form as the corresponding probabilities derived above with the changed sign of $\delta$ and the amplitudes computed for the opposite sign of the potential.

Thus, the $\delta$ dependent terms in all channels are expressed in terms of two combinations of the propagation basis amplitudes, $\left|A_{e \tilde{2}} A_{e \tilde{3}}\right|$ and $D_{23}$. In the case of the maximal 2-3 mixing $\left(D_{23}=0\right)$, only the first combination enters the interference terms. Furthermore, for the channels involving electron neutrinos, $\nu_{\mu}-\nu_{e}$ and $\nu_{\tau}-\nu_{e}$, only the first combination is relevant, even for the non-maximal 2-3 mixing. For generic values of $\delta$ the CP-dependent terms in all the channels but $\nu_{e}-\nu_{e}$ are of the same order.

To assess the $\delta$-dependent interference terms, one can consider the difference of the oscillation probabilities for two different values of the CP-phase:

$$
\Delta P_{\alpha \beta}^{\mathrm{CP}}(\delta) \equiv P_{\alpha \beta}(\delta)-P_{\alpha \beta}\left(\delta_{0}\right)
$$

In practice this would correspond to fit of the probability with the true value of the phase $\delta$ by the probability with some assumed value of the phase $\delta_{0}$. In Figs. 8,10 and 11 we show some examples of the oscillograms for $\Delta P_{\mu e}^{\mathrm{CP}}$ and $\Delta P_{\mu \mu}^{\mathrm{CP}}$ for different values of $\delta$ and $\delta_{0}=0^{\circ}$. Although the CP-oscillograms appear to have a complex structure, this structure can be readily understood in terms of the three grids of curves, which we consider next.

\section{2 "Magic" lines and interference phase lines}

Let us first consider the $\nu_{\mu} \rightarrow \nu_{e}$ oscillation probability, for which the equality

$$
\Delta P_{\mu e}^{\mathrm{CP}}(\delta) \equiv P_{\mu e}(\delta)-P_{\mu e}\left(\delta_{0}\right)=P_{\mu e}^{\delta}(\delta)-P_{\mu e}^{\delta}\left(\delta_{0}\right)
$$

is exact. The condition $\Delta P_{\mu e}^{\mathrm{CP}}=0$ is equivalent to

$$
\left|A_{e \tilde{2}} A_{e \tilde{3}}\right| \cos (\phi-\delta)=\left|A_{e \tilde{2}} A_{e \tilde{3}}\right| \cos \left(\phi-\delta_{0}\right) .
$$

The same condition holds for the $\nu_{e}-\nu_{\tau}$ channel. This equality is satisfied if at least one of the following three conditions is fulfilled

$$
\begin{aligned}
& \text { (A) } A_{e \tilde{2}}\left(E_{\nu}, \Theta_{\nu}\right)=0 \\
& \text { (B) } A_{e \tilde{3}}\left(E_{\nu}, \Theta_{\nu}\right)=0 \\
& \text { (C) } \quad \phi\left(E_{\nu}, \Theta_{\nu}\right)-\delta_{0}=-\left[\phi\left(E_{\nu}, \Theta_{\nu}\right)-\delta\right]+2 \pi l .
\end{aligned}
$$

The last condition implies

$$
\phi\left(E_{\nu}, \Theta_{\nu}\right)=\left(\delta+\delta_{0}\right) / 2+\pi l .
$$


Under conditions (A) and (B) the equality (4.22) is satisfied identically for all values of $\delta$. In these cases the transition probability does not depend on CP-phase. It is also satisfied trivially if $\delta=\delta_{0}+2 \pi n$, since the true and assumed values of the phase coincide. ${ }^{2}$

In general, the conditions (A) and (B) can be satisfied at isolated points in the $\left(\Theta_{\nu}, E_{\nu}\right)$ plane only. Indeed, in order for $A_{e \tilde{3}}$ to vanish, both its real and imaginary parts must be zero. The conditions $\operatorname{Re} A_{e \tilde{3}}=0$ and $\operatorname{Im} A_{e \tilde{3}}=0$ each define a set of curves in the $\left(\Theta_{\nu}, E_{\nu}\right)$ plane, and the curves from one set can only intersect with those from the other at isolated points. The same applies to the condition $A_{e \tilde{2}}=0$. In contrast to this, as we will discussed below, in the factorization approximation both the conditions $A_{e \tilde{2}}=A_{S}=0$ and $A_{e \tilde{3}}=A_{A}=0$ are fulfilled along certain curves in the oscillograms. This happens because the amplitudes $A_{S}$ and $A_{A}$ take a 2 -flavor form. In the bases where the corresponding $2 \times 2$ Hamiltonians are traceless, both $A_{A}$ and $A_{S}$ are pure imaginary because of the symmetry of the Earth's density profile [11]. Therefore for $A_{S}$ and $A_{A}$ to be zero, it is enough to require that their imaginary parts vanish. So, instead of conditions (A) and (B) in (4.23) we will consider equalities $A_{S}=0$ and $A_{A}=0$.

In the factorization approximation the conditions in (A), (B) and (C) define three sets of curves in the oscillograms (see Fig. 8), which play crucial role in understanding effects of $\mathrm{CP}$ violation. Along the lines determined by (A) and (B) the probabilities $P_{\mu e}, P_{e \mu} P_{\tau e}$ and $P_{e \tau}$ do not depend on the CP-phase. The other probabilities (as we will discuss later) only weakly depend on the phase along these lines. The lines shown in Fig. 8 were calculated in the factorization approximation, without assuming constant-density matter, by solving numerically the corresponding 2-flavor evolution problems with the PREM Earth density profile.

In what follows we will consider these lines and their connection to the conditions in Eqs. (4.23) in turn.

\section{- Solar magic lines}

Let us discuss the condition $A_{S}\left(E_{\nu}, \Theta_{\nu}\right)=0$. Notice that at $A_{e \tilde{2}} \approx A_{S}=0$ the "solar" contribution to the amplitudes of the $\nu_{\mu} \leftrightarrow \nu_{e}$ and $\nu_{\tau} \leftrightarrow \nu_{e}$ transitions vanishes, and

$$
P_{\mu e}=P_{e \mu}=s_{23}^{2}\left|A_{e \tilde{3}}\right|^{2}, \quad P_{\tau e}=P_{e \tau}=c_{23}^{2}\left|A_{e \tilde{3}}\right|^{2} .
$$

In Fig. 8 the condition $A_{S}=0$ determines nearly vertical lines at the values of the nadir angle $\Theta_{\nu} \approx 54^{\circ}, 30^{\circ}$ and $12^{\circ}$. This feature can be immediately understood using the constant density approximation. Indeed, according to (2.41) the condition $A_{S}=0$ is fulfilled when

$$
\sin \phi_{S}\left(E_{\nu}, \Theta_{\nu}\right)=0 .
$$

As follows from (2.42), Eq. (4.26) is satisfied when

$$
L\left(\Theta_{\nu}\right) \approx \frac{4 E_{\nu} \pi n}{\Delta m_{21}^{2} \sqrt{\left(\cos 2 \theta_{12} \mp 2 V E_{\nu} / \Delta m_{21}^{2}\right)^{2}+\sin ^{2} 2 \theta_{12}}}, \quad n=1,2, \ldots
$$

\footnotetext{
${ }^{2}$ In Figs. 8, 10 and 11 we show the lines of the condition $(\mathrm{C})$ which correspond to certain values of phases, which we denote by $\left(\delta+\delta_{0}\right)_{\text {fig. }}$. According to (4.13) the interference term vanishes if $\phi=\delta+\pi / 2+\pi n$. Comparing this last equality with (4.24), we find that along the lines of the condition (C) the interference term vanishes for value of phase $\delta=-\left(\delta+\delta_{0}\right)_{\text {fig }} / 2+\pi / 2+\pi n$.
} 


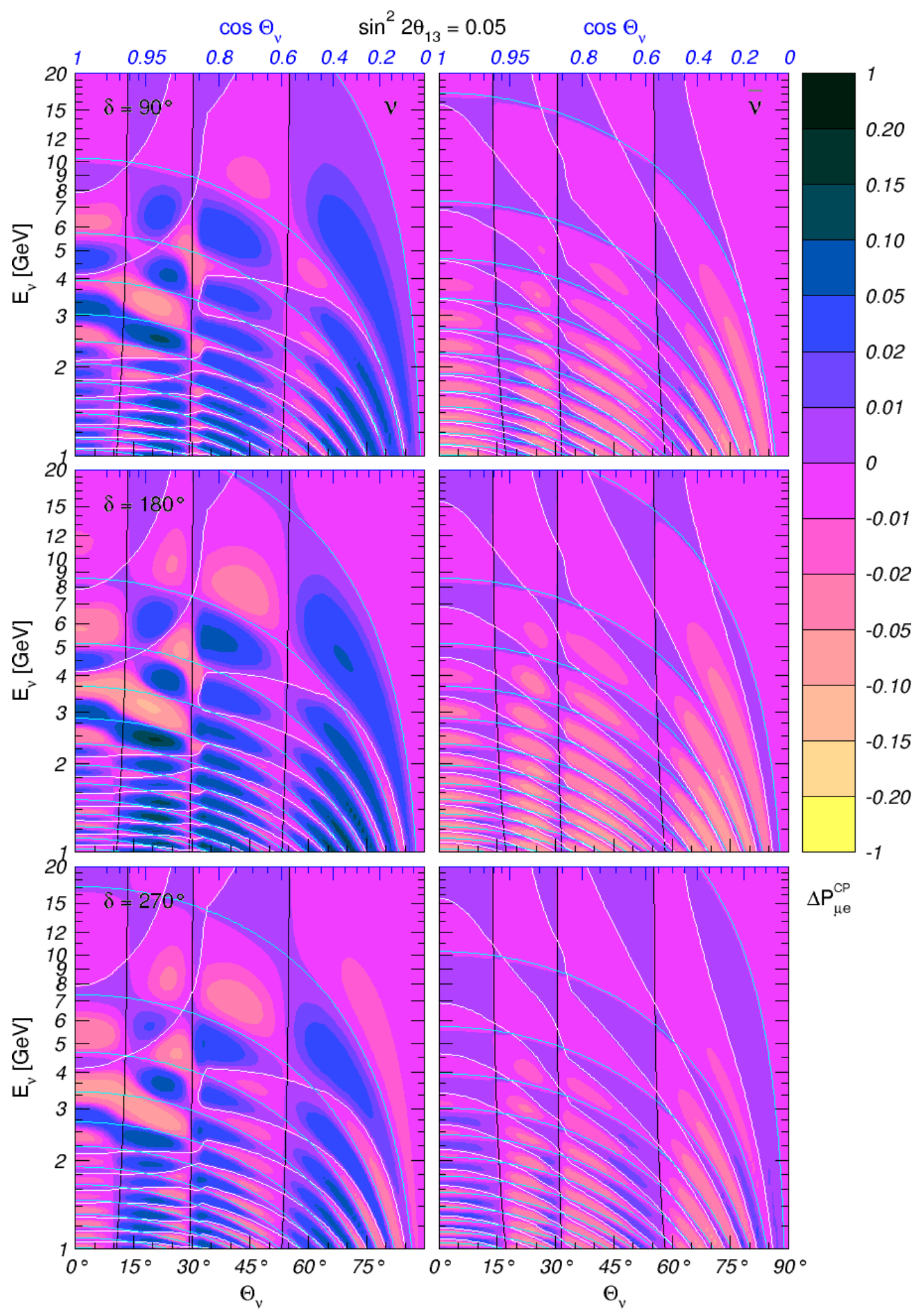

Figure 8: Oscillograms for the difference of probabilities $\Delta P_{\mu e}^{\mathrm{CP}}(\delta)=P_{\mu e}(\delta)-P_{\mu e}\left(\delta_{0}\right)$ with $\delta_{0}=0^{\circ}$. Shown are the solar (black), atmospheric (white) and interference phase condition (cyan) curves. 
Furthermore, at energies that are much higher than the solar MSW resonance energies in the mantle and in the core of the Earth, $E_{\nu} \gtrsim 0.5 \mathrm{GeV}$, the condition (4.27) becomes

$$
L\left(\Theta_{\nu}\right) \simeq \frac{2 \pi n}{V} .
$$

Note that it is energy independent and determines the baselines for which the "solar" contribution to the probability vanishes. In the plane $\left(\Theta_{\nu}, E_{\nu}\right)$ it represents nearly vertical lines $\Theta_{\nu} \approx$ const.

There are three solar magic lines which correspond to $n=1$ (in the mantle domain) and $n=2,3$ (in the core domain). The existence of a baseline ( $L \approx 7600 \mathrm{~km})$ for which the probability of $\nu_{e} \leftrightarrow \nu_{\mu}$ oscillations in the Earth is approximately independent of the "solar" parameters $\left(\Delta m_{21}^{2}, \theta_{12}\right)$ and of the CP-phase $\delta$ was first pointed out in [62] and later discussed in a number of publications (see, e.g., Refs. [63, 64, 15, 65, 66, 61, 67]). This baseline was dubbed "magic" in [63]. The interpretation of this baseline as corresponding to vanishing "solar" amplitude $A_{e \tilde{2}}$, according to Eq. (4.28) with $n=1$, was given in [61]. In [61] it was also shown that for neutrino trajectories crossing the core of the Earth there exist two more solar "magic" baselines, corresponding to the oscillation phase equal $\pi n$ with $n=2$ and 3, and the existence of the atmospheric "magic curves" was pointed out. The three solar "magic" baselines, for which the amplitude $A_{e \tilde{2}}$ vanishes, can be clearly seen in the left panels of Fig. 1.

In the 1-3 resonance region and above, the factorization approximation becomes invalid since the angle $\theta_{13}^{m}$ is large. As a result, $A_{e \tilde{2}}$ and $A_{S}$ become substantially different. Indeed in the constant density approximation, Eq. (2.26), the equality $A_{e \tilde{2}}=0$ is satisfied when

$$
\sin \phi_{21}^{m}\left(E_{\nu}, \Theta_{\nu}\right)=0
$$

For $E_{\nu} \ll E_{13}^{R}$ we have $\phi_{21}^{m} \approx \phi_{S}$. But for energies of the 1-3 resonance and above $\phi_{21}^{m} \neq \phi_{S}$. In particular, for energies substantially above the resonance energy, $\phi_{21}^{m} \approx \phi_{A}^{0}$, and according to Eq. (2.46),

$$
L\left(\Theta_{\nu}\right) \approx \frac{4 E_{\nu} \pi n}{\Delta m_{31}^{2}} .
$$

Thus, in the 1-3 resonance region, the condition $\sin \phi_{S} \approx 0$ transforms into $\sin \phi_{A}^{0}=0$, and the lines of condition $A_{e \tilde{2}} \approx 0$ bend. This can be seen in Figs. 8, where the solar magic lines substantially deviate from the lines of $\Delta P_{\mu e}^{\mathrm{CP}}=0$.

In the antineutrino channel no level crossing occurs and $\phi_{21}^{m} \approx \phi_{S}$ everywhere.

\section{- Atmospheric magic lines}

The atmospheric magic lines are determined by the condition $A_{A}\left(E_{\nu}, \Theta_{\nu}\right)=0$. When the condition $A_{e \tilde{3}}\left(E_{\nu}, \Theta_{\nu}\right) \approx A_{A}\left(E_{\nu}, \Theta_{\nu}\right)=0$ is satisfied, the "atmospheric" contribution to the amplitudes of $\nu_{\mu} \leftrightarrow \nu_{e}$ and $\nu_{\tau} \leftrightarrow \nu_{e}$ transitions vanishes. In this case, too, there are no effects of CP phase on the probabilities of oscillations involving $\nu_{e}$ or $\bar{\nu}_{e}$.

The properties of atmospheric magic lines can be easily understood in the constant density approximation. As follows from Eq. (2.41), the condition $A_{A}=0$ is satisfied when 
$\sin \phi_{A}=0\left(\phi_{A}=\pi k, k=1,2, \ldots\right)$ or explicitly

$$
L\left(\Theta_{\nu}\right) \approx \frac{4 E_{\nu} \pi k}{\Delta m_{31}^{2} \sqrt{\left(\cos 2 \theta_{13} \mp 2 V E_{\nu} / \Delta m_{31}^{2}\right)^{2}+\sin ^{2} 2 \theta_{13}}}, \quad k=1,2, \ldots
$$

For energies which are not too close to the atmospheric MSW resonance energy, the condition (4.31) reduces to

$$
E_{\nu} \simeq \frac{\Delta m_{31}^{2} L\left(\Theta_{\nu}\right)}{\left|4 \pi k \pm 2 V L\left(\Theta_{\nu}\right)\right|},
$$

which corresponds to the bent curves in the $\left(\Theta_{\nu}, E_{\nu}\right)$ plane. For very large energies, where $\Delta m_{31}^{2} / 2 E \ll V$, the atmospheric lines approach the same vertical lines as the solar magic lines (4.28).

Let us now consider the condition $A_{e \tilde{3}}\left(E_{\nu}, \Theta_{\nu}\right)=0$. In the constant density approximation it gives, according to (2.27),

$$
\sin \phi_{32}^{m}=-e^{i \phi_{31}^{m}} \cos ^{2} \theta_{12}^{m} \sin \phi_{21}^{m} .
$$

In turn, Eq. (4.33) implies two conditions which follow from the real and imaginary parts of the equality:

$$
\begin{gathered}
\sin \phi_{32}^{m}=-\cos \phi_{31}^{m} \cos ^{2} \theta_{12}^{m} \sin \phi_{21}^{m}, \\
\sin \phi_{31}^{m} \sin \phi_{21}^{m}=0 .
\end{gathered}
$$

Both conditions can be satisfied simultaneously only at certain points of the parameter space, which illustrates our general statement in the beginning of this subsection. However at high energies $\cos ^{2} \theta_{12}^{m} \ll 1$, and the equality (4.33) approximately reduces to

$$
\sin \phi_{32}^{m}=0 \text {. }
$$

Furthermore, since $\phi_{32}^{m} \approx \phi_{A}$ in the energy range above the 1-2 resonance, in this channel the factorization approximation works well. The atmospheric magic lines reproduce very well the lines of $\Delta P_{\mu e}^{\mathrm{CP}}=0$. No interconnection of the atmospheric magic lines occurs.

\section{- The interference phase condition}

Let us now find the lines in the $\left(\Theta_{\nu}, E_{\nu}\right)$ plane which correspond to the interference phase condition (4.24). We shall call these lines the interference phase lines. Consider the condition (4.24) in the factorization approximation. Although $A_{e \tilde{2}}$ and $A_{e \tilde{3}}$ are pure imaginary in the bases where their respective $2 \times 2$ Hamiltonians are traceless, their relative complex phase $\phi$ in any fixed basis is different from zero. It just equals the rotation phase between the aforementioned 2 -flavor bases. In the constant density approximation (3.27) $\phi \approx-\phi_{31}^{m}$. In the energy range between the two resonances we have

$$
\phi_{31}^{m} \approx \frac{\Delta m_{31}^{2} L}{4 E_{\nu}}=\phi_{A}^{0},
$$

i.e., in the first approximation $\phi$ does not depend on the matter density. From (4.24) we then obtain

$$
\frac{\Delta m_{31}^{2} L}{4 E_{\nu}}=-\frac{\delta+\delta_{0}}{2}+\pi l,
$$




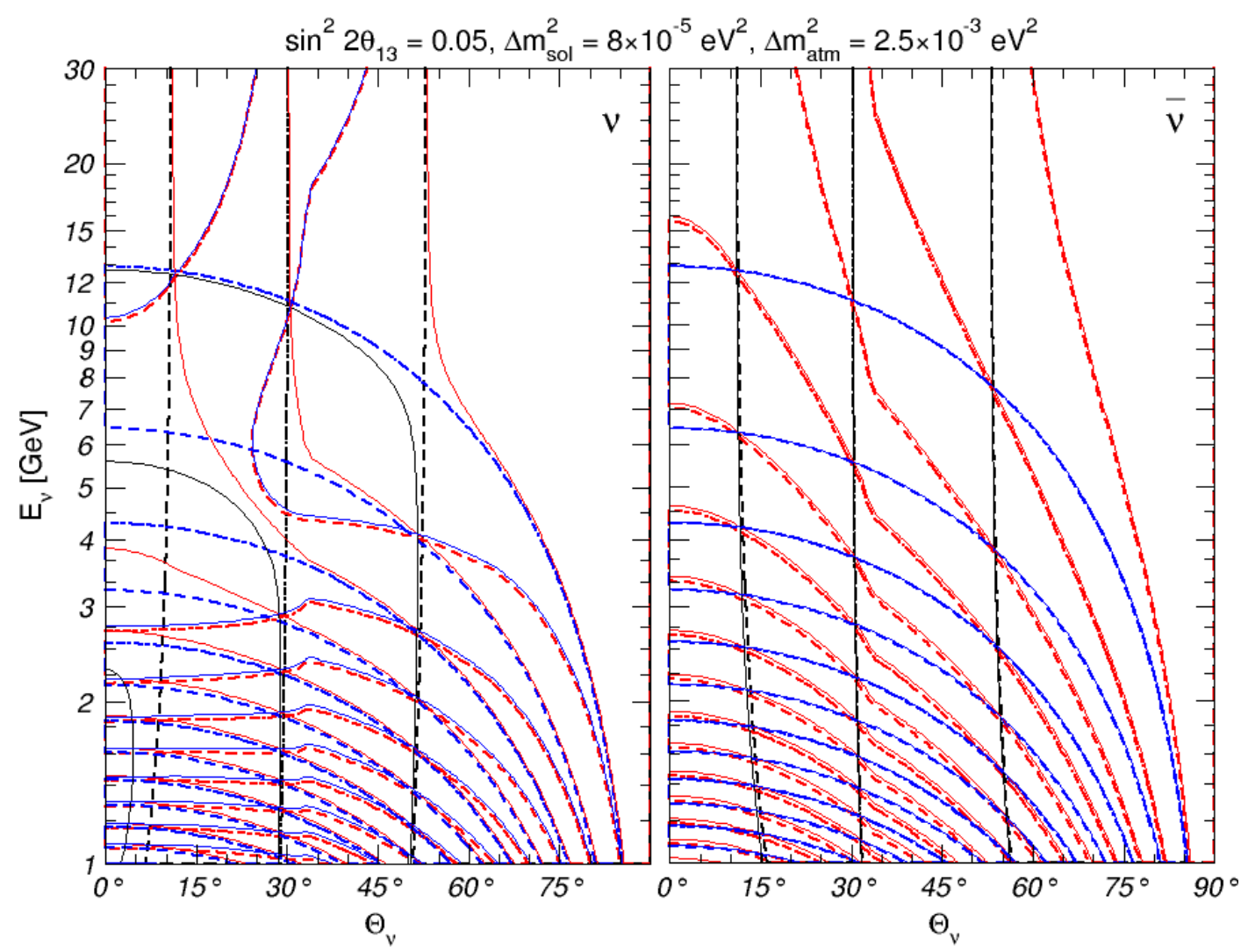

Figure 9: Grids of lines along which $\sin \phi_{i}=0$ for different adiabatic phases $\phi_{i}$. Dashed lines correspond to $\phi_{S}$ (black), $\phi_{A}$ (red) and $\phi_{A}^{0}$ (blue) obtained in $2 \nu$ context. Solid lines correspond to $3 \nu$ calculations: $\phi_{21}^{m}$ (black), $\phi_{31}^{m}$ (red) and $\phi_{32}^{m}$ (blue).

or

$$
E_{\nu}=\frac{\Delta m_{31}^{2} L\left(\Theta_{\nu}\right)}{4 \pi l-2\left(\delta+\delta_{0}\right)} .
$$

This gives rather accurate description of the lines $\Delta P_{\mu e}^{\mathrm{CP}}=0$ below the 1-3 resonance.

In the $3 \nu$ framework $\phi_{31}^{m}$ differs from $\phi_{A}^{0}$ in the $1-3$ resonance region. Above the $1-3$ resonance $\phi_{31}^{m} \approx \phi_{S}$, and as we discussed before, the lines of $\phi_{S}=$ const (Eq. (4.28)) depend on energy. Hence, the interference phase lines become nearly vertical with increasing energy, as can be seen in Fig. 9 .

Summarizing, the phase $\phi_{21}^{m}$ that enters into the amplitude $A_{e \tilde{2}}$ nearly coincides with $\phi_{S}$ below the 1-3 resonance. However, above the 1-3 resonance it approaches the vacuum phase $\phi_{A}^{0}$. In turn, the interference phase $\phi \approx \phi_{31}^{m}$, which approximately coincides with $\phi_{A}^{0}$ between the 1-2 and 1-3 resonances, approaches $\phi_{S}$ with increase of energy above the 1-3 resonance. The lines $\sin \phi_{S}=0$ and $\sin \phi_{A}^{0}=0$ cross, whereas $\sin \phi_{21}^{m}=0$ and $\sin \phi_{31}^{m}=0$ do not. Thus, in the region of the 1-3 resonance, where the factorization approximation is strongly broken in the 1-2 channel, interconnections of the lines occur: the contours of zero $\Delta P_{\mu e}^{\mathrm{CP}}$ transform (interpolate) from the solar magic lines to the interference phase lines and vice versa. The interconnection is related to the level crossing phenomenon and reflects the level crossing scheme, it reflects the described change of the phases $\phi_{21}^{m}$ and $\phi_{31}^{m}$. To 
illustrate this effect explicitly, we show in Fig. 9 the lines $\sin \phi_{S}=0$ and $\sin \phi_{A}=0$, which represent the magic lines (defined in the $2 \nu$ context), and the lines $\sin \phi_{21}^{m}=0, \sin \phi_{31}^{m}=0$ and $\sin \phi_{32}^{m}=0$ which represent the conditions of vanishing amplitudes in the $3 \nu$ context, that is, the contours of vanishing $\Delta P_{\mu e}^{\mathrm{CP}}$. Clearly, they do not coincide with the contours $\Delta P_{\mu e}^{\mathrm{CP}}=0$, since we have taken into account the phase factors only. This grid reproduces qualitatively well all the features of the lines shown in Fig. 8.

There is no level crossing in the antineutrino channels for the normal mass hierarchy, and therefore there is no interconnection of the lines there.

\subsection{CP-phase domains for channels involving $\nu_{e}$}

The solar and atmospheric "magic" lines and the interference phase curves allow a simple interpretation of the CP oscillograms. The solar (nearly vertical) and atmospheric (bent) curves divide the oscillograms into a set of domains, which are in turn divided by the grid of the interference phase curves into sub-domains (see Fig. 8). From these figures one can see that the interference phase curves are steeper than the atmospheric curves in the case of neutrinos and less steep than atmospheric curves for antineutrinos, in full agreement with Eqs. (4.32) and (4.39). This fact is related with the sign in Eq. (4.32). The probability difference $\Delta P_{\mu e}^{\mathrm{CP}}(\delta)$ vanishes at the borders of these sub-domains: On the solar and atmospheric "magic" curves because the probabilities are $\delta$-independent there, and on the interference phase curves because they correspond to $\cos (\phi-\delta)=\cos \left(\phi-\delta_{0}\right)$. The signs of the probability differences in the neighboring sub-domains are opposite, with the maxima of the difference in the central parts of sub-domains.

As can be seen from Fig. 8, with changing the true (or assumed) values of $\delta$, the solar and atmospheric grids remain unchanged, whereas the grid of the interference phase curves moves up or down, in accord with Eqs. (4.28), (4.32) and (4.39). The constant-density approximation results of Eqs. (4.28), (4.32) and (4.39) reproduce the main features of these curves quite well.

As can be seen from the figures, the borders between the regions of the positive and negative CP-phase effect do not coincide exactly with the magic lines, especially in the regions of intersection of these lines. This indicates deviation from the factorization approximation and is related to the level crossing phenomenon, as we have discussed in the previous subsection.

\subsection{CP-domains for channels not involving $\nu_{e}$}

As follows from our consideration in Sec. 4.1, the $\delta$-dependent parts of the probabilities for the channels which do not contain $\nu_{e}$ have more complicated structure than those which do. Apart from the term proportional the product of the amplitudes $A_{e \tilde{2}} A_{e \tilde{3}}^{*}$ they contain contributions proportional to the deviation of the 2-3 mixing from the maximal one as well as terms $P^{\delta \delta}$ proportional to the square of the small amplitude $A_{\tilde{2} \tilde{3}}$. In what follows we will neglect the latter.

In the case of the maximal 2-3 mixing the $\delta$-dependent parts of the probabilities are

given in Eqs. (4.14) and (4.15). Furthermore, $P_{\tau \tau}^{\delta}=-P_{\mu \mu}^{\delta}$ and $P_{\tau \mu}^{\delta}=-P_{\mu \tau}^{\delta}$. From Eq. (4.14) it follows that for $\theta_{23}=45^{\circ}$ the CP-oscillograms for the survival probability $P_{\mu \mu}$ 


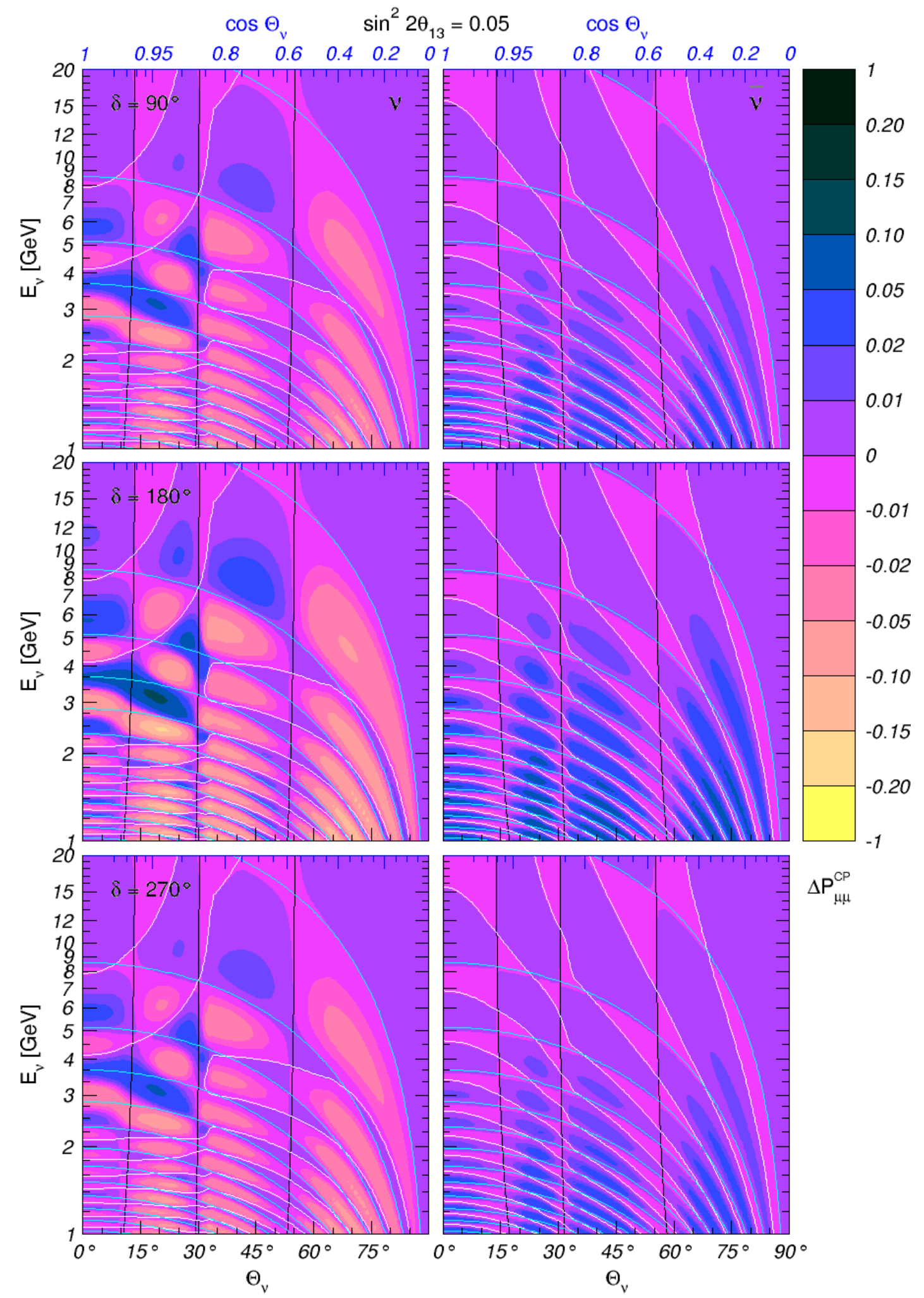

Figure 10: Oscillograms for the difference of probabilities $\Delta P_{\mu \mu}^{\mathrm{CP}}(\delta)=P_{\mu \mu}(\delta)-P_{\mu \mu}\left(\delta_{0}\right)$ with $\delta_{0}=0^{\circ}$. Shown are the solar (black), atmospheric (white) and interference phase condition (cyan) curves. 
can be interpreted in terms of the same grids of the solar and atmospheric "magic" curves $A_{S}=0$ and $A_{A}=0$ that we used for the analysis of the oscillograms for $P_{\mu e}$ and $P_{\tau e}$ (see Fig. 10).

The key difference from the previous case is that now the CP-phase and the interference phase dependencies factorize. In the interference terms $P_{\mu \mu}^{\delta}$ and $P_{\tau \tau}^{\delta}$ they appear as $\cos \delta \cos \phi$. Therefore, the third grid describing oscillograms in these channels consists of the interference phase curves

$$
\phi \approx-\phi_{31}^{m}=\frac{\pi}{2}+\pi n,
$$

which, unlike the interference phase curves for the $\nu_{\mu}-\nu_{e}$ and $\nu_{\tau}-\nu_{e}$ channels, do not depend on the values of $\delta$ and $\delta_{0}$. The difference between the probabilities calculated with the true and assumed values of $\delta$ (as well as the effects of $\delta$ in general) vanishes on the curves of all three types and take maximum values in the central parts the domains delimited by these curves. The borders of the domains do not move with change of $\delta$, and the only change that happens is that within each domain the probability varies proportionally to $\cos \delta$.

The $\delta$ dependent terms of the transition probabilities, $P_{\mu \tau}^{\delta}$ and $P_{\tau \mu}^{\delta}$ are proportional to $\sin \delta \sin \phi$. Therefore for these probabilities the interference phase condition reads

$$
\phi \approx \phi_{31}^{m}=\pi n
$$

Again the borders of the domains do not depend on $\delta$ and with changing $\delta$ the interference terms vary as $\sin \delta$.

Notice that the borders of domains are rather stable with respect to variations of the neutrino parameters. Since the grids are determined mainly by the phases, their dependence on the 1-3 mixing is weak. With the decrease of the 1-3 mixing angle in vacuum the grid lines becomes closer to the lines of vanishing $\Delta P_{\mu e}^{\mathrm{CP}}$. Since $\theta_{23}$ is experimentally known to be rather close to $45^{\circ}$, the discussed solar, atmospheric and interference phase curves give a rather good description of the CP-oscillograms for $P_{\mu \mu}$ even when $\theta_{23}$ deviates from the maximal-mixing value (see Fig. 11). ${ }^{3}$

\subsection{Sensitivity to the CP phase in the $\nu_{\mu}-\nu_{e}$ channel}

Let us now identify the regions of the experimental parameters $\Theta_{\nu}$ and $E_{\nu}$ for which the oscillation probabilities have maximal sensitivity to the CP phase $\delta$.

Consider the variation of the oscillation probabilities with varying $\delta$ while all the other oscillation parameters are fixed. As follows from Eq. (4.8), the maximal variation of the probability $P_{\mu e}$ with $\delta$ changing between $0^{\circ}$ and $360^{\circ}$ is

$$
\Delta P_{\mu e}^{\max } \equiv \max \left[P_{\mu e}(\delta)\right]-\min \left[P_{\mu e}(\delta)\right]=2 \sin 2 \theta_{23}\left|A_{e \tilde{2}} A_{e \tilde{3}}\right| \cdot
$$

(Note that a similar quantity was considered in [68]). This quantity is maximized when $\left|A_{e \tilde{2}} A_{e \tilde{3}}\right|$ takes the maximum possible value. Let us now discuss the dependence of $\left|A_{e \tilde{2}} A_{e \tilde{3}}\right|$

\footnotetext{
${ }^{3}$ Actually, the correction due to $\theta_{23} \neq 45^{\circ}$ is of the order $\frac{1}{2} \cos 2 \theta_{23} \lesssim 0.15$.
} 


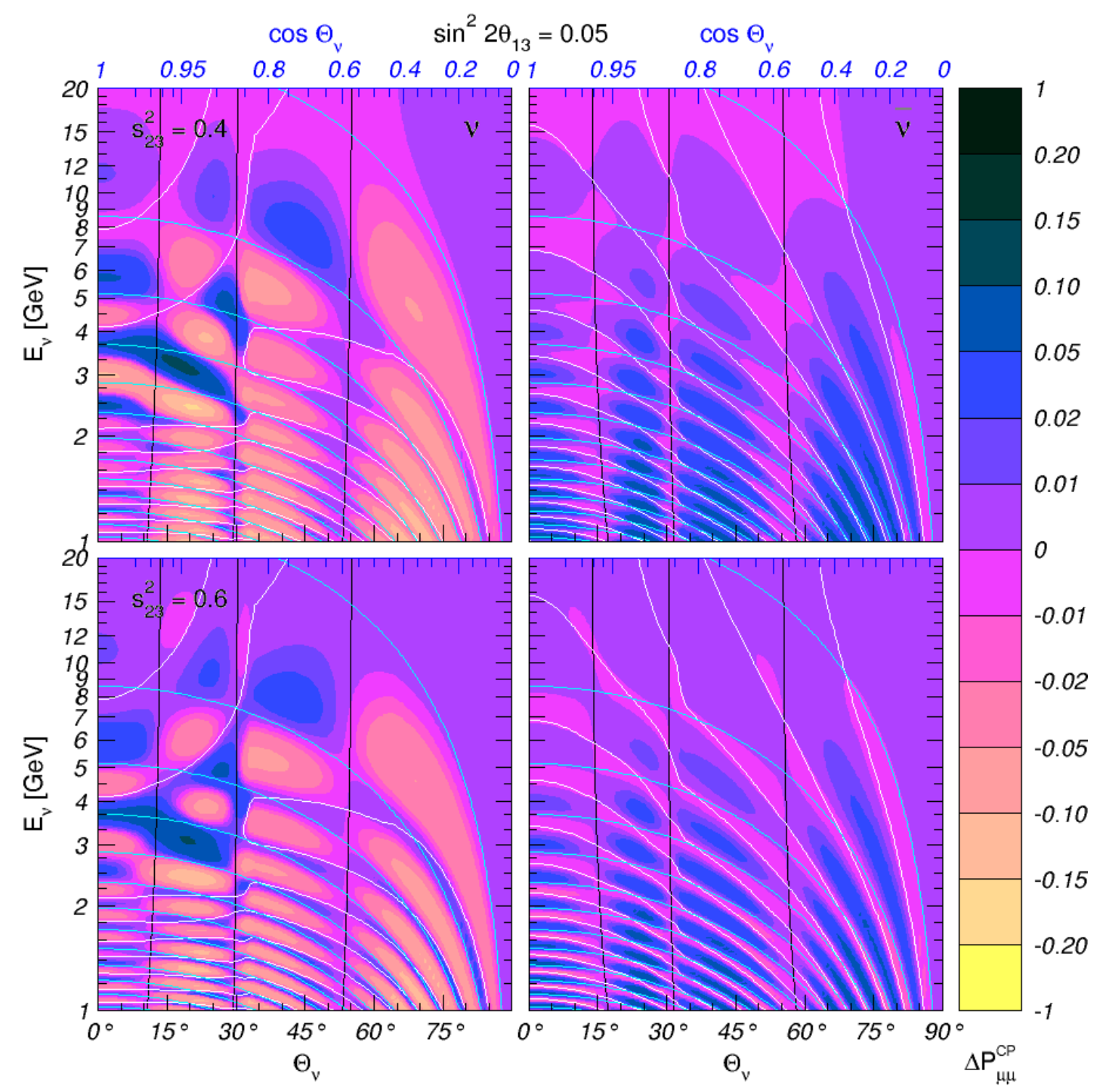

Figure 11: Contour plots for the probability difference $\Delta P_{\mu \mu}^{\mathrm{CP}}(\delta)=P_{\mu \mu}(\delta)-P_{\mu \mu}\left(\delta_{0}\right)$ with $\delta_{0}=0^{\circ}$ and $\delta=180^{\circ}$. Upper panels: $s_{23}^{2}=0.4$, lower panels: $s_{23}^{2}=0.6$. Shown are the solar (black), atmospheric (white) and interference phase (cyan) curves.

on the experimental parameters $\Theta_{\nu}$ and $E_{\nu}$ in the factorization approximation. For mantleonly crossing trajectories it is sufficient to use the constant-density matter factorization approximation, in which

$$
\left|A_{e \tilde{2}}\right|=\sin 2 \theta_{12}^{m}\left|\sin \phi_{S}\right|, \quad\left|A_{e \tilde{3}}\right|=\sin 2 \theta_{13}^{m}\left|\sin \phi_{A}\right| .
$$

Thus, we have to find the maxima of the quantity

$$
A \equiv \sin 2 \theta_{12}^{m} \sin 2 \theta_{13}^{m}\left|\sin \phi_{21}^{m} \sin \phi_{31}^{m}\right|
$$

with respect to $\Theta_{\nu}$ and $E_{\nu}$. To do this exactly is a rather complicated problem, and the result would be bulky and not easily tractable; fortunately, an approximate maximization 
can be readily carried out by studying the energy dependence of the different factors in (4.44).

First, recall that for energies $E_{\nu} \gtrsim 0.5 \mathrm{GeV}$ the "solar" phase $\phi_{S}$ is essentially energy independent. Therefore $\sin \phi_{S}$ can be considered a constant factor when maximizing $A$ with respect to the neutrino energy. The maximum $\left|\sin \phi_{S}\right|=1$ can be achieved by properly choosing the values of the baseline $L$. These are approximately equal to the central values of $L$ (i.e., of $\cos \Theta_{\nu}$ ) between the solar "magic" lines in the oscillograms of Fig. 8 (note that in the second band, due to the existence of the mantle-core boundary, the maximum is shifted from the center).

Next, consider the extrema of the remaining factor, $\sin 2 \theta_{12}^{m} \sin 2 \theta_{13}^{m}\left|\sin \phi_{A}\right|$, with respect to the neutrino energy for fixed $L$. To do this, we make use of the fact that the function

$$
f\left(E_{\nu}\right) \equiv \sin 2 \theta_{12}^{m} \sin 2 \theta_{13}^{m}
$$

varies with $E_{\nu}$ significantly more slowly than $\sin \phi_{A}$. If $f\left(E_{\nu}\right)$ were constant, the maxima of $A$ with respect to $E_{\nu}$ would coincide with the maxima of $\left|\sin \phi_{A}\right|$; in reality, the (relatively) weak energy dependence of $f\left(E_{\nu}\right)$ on $E_{\nu}$ leads to a slight shift of the exact maxima $A$ from those of $\left|\sin \phi_{A}\right|$. The main effect of the energy dependence of $f\left(E_{\nu}\right)$ is actually to modulate the maxima of $\left|\sin \phi_{A}\right|$. In other words, the maxima of $\Delta P_{\mu e}^{\max }$ nearly coincide with the absolute maxima of $\left|\sin \phi_{S} \sin \phi_{A}\right|$ (equal to 1), which are achieved by a proper choice of the values of the nadir angle and energy; the actual height of the local maxima of $\Delta P_{\mu e}^{\max }$ is determined by the value of $f\left(E_{\nu}\right)$ in these maxima.

This is illustrated by Fig. 12. In the $\Theta_{\nu}$ direction, the maxima of $\Delta P_{\mu e}^{\max }$ correspond to the maxima of $\left|\sin \phi_{S}\right|$. This fixes the values of the baseline $L$. The vertical "domain structure" (i.e., the structure in the energy direction) is due to the oscillatory dependence of $\left|\sin \phi_{A}\right|$. For fixed $L$ the energies $E_{n}$ at which $\Delta P_{\mu e}^{\max }$ has maxima are approximately found from the condition $\left|\sin \phi_{A}\right|=1$, or $\phi_{A} \approx \omega_{31} L=\pi / 2+\pi n$. The heights of these local maxima are determined by $f\left(E_{n}\right)$.

To find out which of the peaks of $\Delta P_{\mu e}^{\max }$ are the highest, one has to consider the maxima of the envelop function $f\left(E_{\nu}\right)$. We will do that here for the resonance channel (neutrinos); the analysis for antineutrinos can be easily performed along the same lines.

The condition $d f / d E_{\nu}=0$ yields the third-order equation

$$
\begin{aligned}
& 2 x^{3}-3 x^{2}\left(\cos 2 \theta_{13}+r_{\Delta} \cos 2 \theta_{12}\right) \\
& \quad+x\left(1+4 r_{\Delta} \cos 2 \theta_{12} \cos 2 \theta_{13}+r_{\Delta}^{2}\right)-r_{\Delta} \cos 2 \theta_{12}-r_{\Delta}^{2} \cos 2 \theta_{13}=0,
\end{aligned}
$$

where $x$ is defined in (3.18). For $\sin ^{2} 2 \theta_{13} \lesssim 1 / 9$ it has three solutions, which correspond to two maxima of $f\left(E_{\nu}\right)$ and a minimum between the two maxima. Expanding in $r_{\Delta}$, we find for the position of the low-energy maximum

$$
x_{1} \simeq r_{\Delta} \cos 2 \theta_{12}+r_{\Delta}^{2} \sin ^{2} 2 \theta_{12} \cos 2 \theta_{13},
$$

or

$$
E_{1} \simeq E_{12}^{R}\left(1+r_{\Delta} \sin 2 \theta_{12} \tan 2 \theta_{12} \cos 2 \theta_{13}\right)
$$




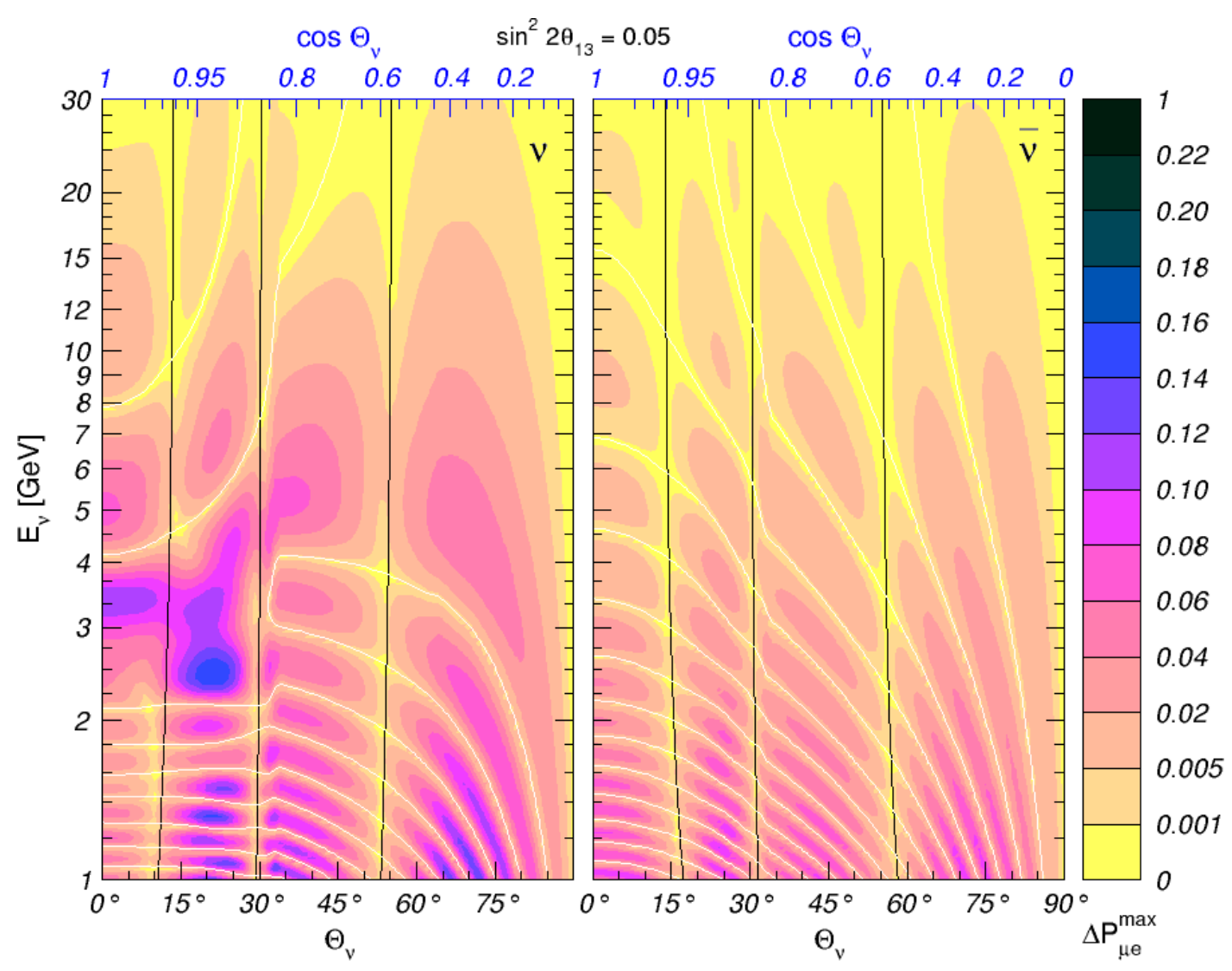

Figure 12: Contour plots for the probability difference $\Delta P_{\mu e}^{\max }=\max P_{\mu e}-\min P_{\mu e}$ for $\delta$ varying between $0^{\circ}$ and $360^{\circ}$ and all the other oscillation parameters fixed. Also shown are the solar (black) and atmospheric (white) magic curves. We set $\Delta m_{21}^{2}=8 \times 10^{-5} \mathrm{eV}^{2}$ and $\sin ^{2} 2 \theta_{13}=0.05$.

Here $E_{12}^{R}$ is the energy of the 1-2 resonance (which corresponds to $x=r_{\Delta} \cos 2 \theta_{12}$ ). Thus, the low-energy maximum of $f\left(E_{\nu}\right)$ practically coincides with the low-energy MSW resonance, and the 1-3 mixing produces only a slight $\left(\mathcal{O}\left(r_{\Delta}\right)\right)$ upward shift of the position of the maximum.

The minimum of $f\left(E_{\nu}\right)$ is given by

$$
x_{2} \simeq \frac{3}{4} \cos 2 \theta_{13}-\frac{1}{4} \sqrt{1-9 \sin ^{2} 2 \theta_{13}},
$$

while the second maximum is at

$$
x_{3} \simeq \frac{3}{4} \cos 2 \theta_{13}+\frac{1}{4} \sqrt{1-9 \sin ^{2} 2 \theta_{13}} .
$$

Recall that the MSW resonance energy in the 1-3 channel corresponds to $x=x_{13}^{R} \equiv \cos 2 \theta_{13}$.

Consider the dependence of $f\left(E_{\nu}\right)$ on $E_{\nu}$ for different values of the 1-3 mixing. For $\theta_{13} \rightarrow 0$ we have $x_{3} \rightarrow 1$ and $x_{2} \rightarrow 1 / 2$, i.e., $E_{3} \rightarrow E_{13}^{R}$ and $E_{2} \rightarrow E_{13}^{R} / 2$. Thus, in this limit the positions of the maxima of $f\left(E_{\nu}\right)$ coincide with the 1-2 and 1-3 MSW resonance energies, while the minimum is approximately in the middle between them.

At maxima, the values of function $f$ can be estimated as $f\left(x_{1}\right) \sim \sin 2 \theta_{13}$ and $f\left(x_{1}\right) \sim$ $r_{\Delta} \sin 2 \theta_{12}$. 
With increasing 1-3 mixing, the minimum of $f\left(E_{\nu}\right)$ shifts to larger energies:

$$
x_{2} \approx \frac{1}{2}+\frac{3}{4} \sin ^{2} 2 \theta_{13}
$$

whereas the second maximum moves to lower energies:

$$
x_{3} \approx 1-\frac{3}{2} \sin ^{2} 2 \theta_{13} \approx x_{13}^{R} \cos ^{2} 2 \theta_{13} .
$$

For $\sin ^{2} 2 \theta_{13}=1 / 9$ one has $x_{2}=x_{3}=1 / \sqrt{2}$, i.e., the minimum and the second maximum of $f\left(E_{\nu}\right)$ merge. This just corresponds to the situation when the local minimum of the cubic function on the l.h.s. of Eq. (4.46) touches the $x$-axis.

For $\sin ^{2} 2 \theta_{13}>1 / 9$ only the low-energy maximum of $f\left(E_{\nu}\right)$ persists. Thus, for these values of $\sin ^{2} 2 \theta_{13}$ the effect of the CP phase is maximal at the 1-2 resonance and decreases with increasing energy. For the other value, $\sin ^{2} 2 \theta_{13}=0.05$ (which in fact is not too small), we find $x_{2} \approx 0.57$ and $x_{3} \approx 0.89$.

These results allow one to readily understand the oscillograms for $\Delta P_{\mu e}^{\max }$ (Fig. 12), at least for mantle-only crossing neutrinos. For $\sin ^{2} 2 \theta_{13}=0.05$ the minimum of $\Delta P_{\mu e}^{\max }$ is situated at $E_{\nu} \sim 3-3.5 \mathrm{GeV}(x \approx 0.57)$, which can be seen in the strip between the first and the second solar magic lines: the peaks increase in height both with energies decreasing and increasing from $E_{\nu} \sim 3-3.5 \mathrm{GeV}$ (in contrast to this, for $\sin ^{2} 2 \theta_{13}=0.125$ the height of the peaks would monotonically decrease with increasing neutrino energy). Note that the situation is somewhat different in the strip between the Earth's surface $\left(\Theta_{\nu}=90^{\circ}\right)$ and the first solar "magic" line: the peak at $E_{\nu} \sim 5 \mathrm{GeV}$ is actually lower than that at $\sim 3 \mathrm{GeV}$ because the baseline is relatively short, and at high energies the oscillation phase $\phi_{A}$ is too small for the condition $\left|\sin \phi_{A}\right|=1$ to be satisfied.

For core-crossing neutrino trajectories the constant-density approximation is not in general applicable, and a different approach is necessary. The maxima of $\Delta P_{\mu e}^{\max }$ in that case can, in principle, be analyzed in the factorization approximation by making use of simple formulas for 2-flavor neutrino evolution in 3-layer matter density profiles obtained in [31]. In Fig. 12 one can see strong enhancement of the difference of amplitudes in the core domain at $E_{\nu} \sim 2.5 \mathrm{GeV}$, which is apparently due to the mantle-core effect.

\subsection{Sensitivity to the CP phase in the $\nu_{\mu}-\nu_{\mu}$ channel}

Let us now discuss the sensitivity of the survival probability $P_{\mu \mu}$ to the phase $\delta$. For other discussions of this issue see, e.g., [52, 54, 55]. From Eq. (2.16) one finds

$$
P_{\mu \mu}=|C+D z|^{2}
$$

where

$$
C=c_{23}^{2} A_{\tilde{2} \tilde{2}}+s_{23}^{2} A_{\tilde{3} \tilde{3}}, \quad D=2 s_{23} c_{23} A_{\tilde{2} \tilde{3}}, \quad z \equiv \cos \delta .
$$

The maximum and minimum values of $P_{\mu \mu}$ with varying $\delta$ then correspond to the maximum and minimum of the modulus of the complex number $C+D z$ when $C$ and $D$ are fixed 
and $z$ is allowed to vary between -1 and 1 . A simple geometrical consideration then shows that for

$$
2 s_{23} c_{23}\left|A_{\tilde{2} \tilde{3}}\right|^{2} \leq\left|\operatorname{Re}\left[A_{\tilde{2} \tilde{3}}^{*}\left(c_{23}^{2} A_{\tilde{2} \tilde{2}}+s_{23}^{2} A_{\tilde{3} \tilde{3}}\right)\right]\right|
$$

the minimum of $P_{\mu \mu}$ corresponds to $\delta=0$ and maximum to $\delta=180^{\circ}$ or vice-versa, so that for $\Delta P_{\mu \mu}^{\max } \equiv \max \left[P_{\mu \mu}(\delta)\right]-\min \left[P_{\mu \mu}(\delta)\right]$ one finds

$$
\begin{aligned}
\Delta P_{\mu \mu}^{\max } & =\left|P_{\mu \mu}\left(\delta=180^{\circ}\right)-P_{\mu \mu}\left(\delta=0^{\circ}\right)\right| \\
& =8 s_{23} c_{23}\left|\operatorname{Re}\left[A_{\tilde{2} \tilde{3}}^{*}\left(c_{23}^{2} A_{\tilde{2} \tilde{2}}+s_{23}^{2} A_{\tilde{3} \tilde{3}}\right)\right]\right| .
\end{aligned}
$$

If, on the contrary,

$$
2 s_{23} c_{23}\left|A_{\tilde{2} \tilde{3}}\right|^{2}>\left|\operatorname{Re}\left[A_{\tilde{2} \tilde{3}}^{*}\left(c_{23}^{2} A_{\tilde{2} \tilde{2}}+s_{23}^{2} A_{\tilde{3} \tilde{3}}\right)\right]\right|,
$$

then for the maximal variation of $P_{\mu \mu}$ with $\delta$ one finds

$$
\Delta P_{\mu \mu}^{\max }=\left(\frac{\left|\operatorname{Re}\left[A_{\tilde{2} \tilde{3}}^{*}\left(c_{23}^{2} A_{\tilde{2} \tilde{2}}+s_{23}^{2} A_{\tilde{3} \tilde{3}}\right)\right]\right|}{\left|A_{\tilde{2} \tilde{3}}\right|}+2 s_{23} c_{23}\left|A_{\tilde{2} \tilde{3}}\right|\right)^{2} .
$$

Note that $A_{\tilde{2} \tilde{3}}$ is a small quantity, so that the condition (4.55) is satisfied in most of the parameter space. Exceptions are the regions where the "main" contribution to $\Delta P_{\mu \mu}^{\max }$, i.e., $\left|c_{23}^{2} A_{\tilde{2} \tilde{2}}+s_{23}^{2} A_{\tilde{3} \tilde{3}}\right|^{2}$, is anomalously small, that is the regions along the magic lines. This is illustrated by Fig. 13, where we show the oscillograms for the maximum probability differences $\Delta P_{\mu \mu}^{\max }$ for $\delta$ varying between $0^{\circ}$ and $360^{\circ}$. The areas corresponding to the regions where the condition (4.55) is not satisfied occupy a rather small fraction of the parameter space. Moreover, $\Delta P_{\mu \mu}^{\max }$ is small in these regions, so that they correspond to low sensitivity to the effects of the $\mathrm{CP}$ phase. Note that these regions would never appear if one neglected the term of the order of $\left|A_{\tilde{2} \tilde{3}}\right|^{2}$ in the expression for $P_{\mu \mu}$.

For the regions where the condition (4.55) is satisfied, one can obtain a simple expression for $\Delta P_{\mu \mu}^{\max }$ in the limit $\theta_{23}=45^{\circ}$. From Eqs. (4.56) and (4.1) one then finds

$$
\Delta P_{\mu \mu}^{\max }=2\left|\operatorname{Re}\left(A_{e \tilde{2}} A_{e \tilde{3}}^{*}\right)\right| .
$$

Thus, for $\theta_{23}=45^{\circ}$ the oscillograms for the maximum probability difference $\Delta P_{\mu \mu}^{\max }$ are also governed by the solar and atmospheric "magic" curves, as well as by the interference phase curves (4.40), in full accord with our discussion in Sec. 4.2.

\section{Discussion and conclusions}

The main purpose of the present paper is to gain a physics insight into the complex pattern of full 3-flavor neutrino oscillations in the Earth. To this end, we presented a detailed description of the three-flavor neutrino oscillation effects in the Earth in terms of the neutrino oscillograms, i.e., contours of equal oscillation probabilities or probability differences in the neutrino nadir angle - energy plane.

We have found that for very small or vanishing 1-3 mixing the oscillation pattern appears in the low energy region with large (maximal) transition probabilities below $0.3 \mathrm{GeV}$. 


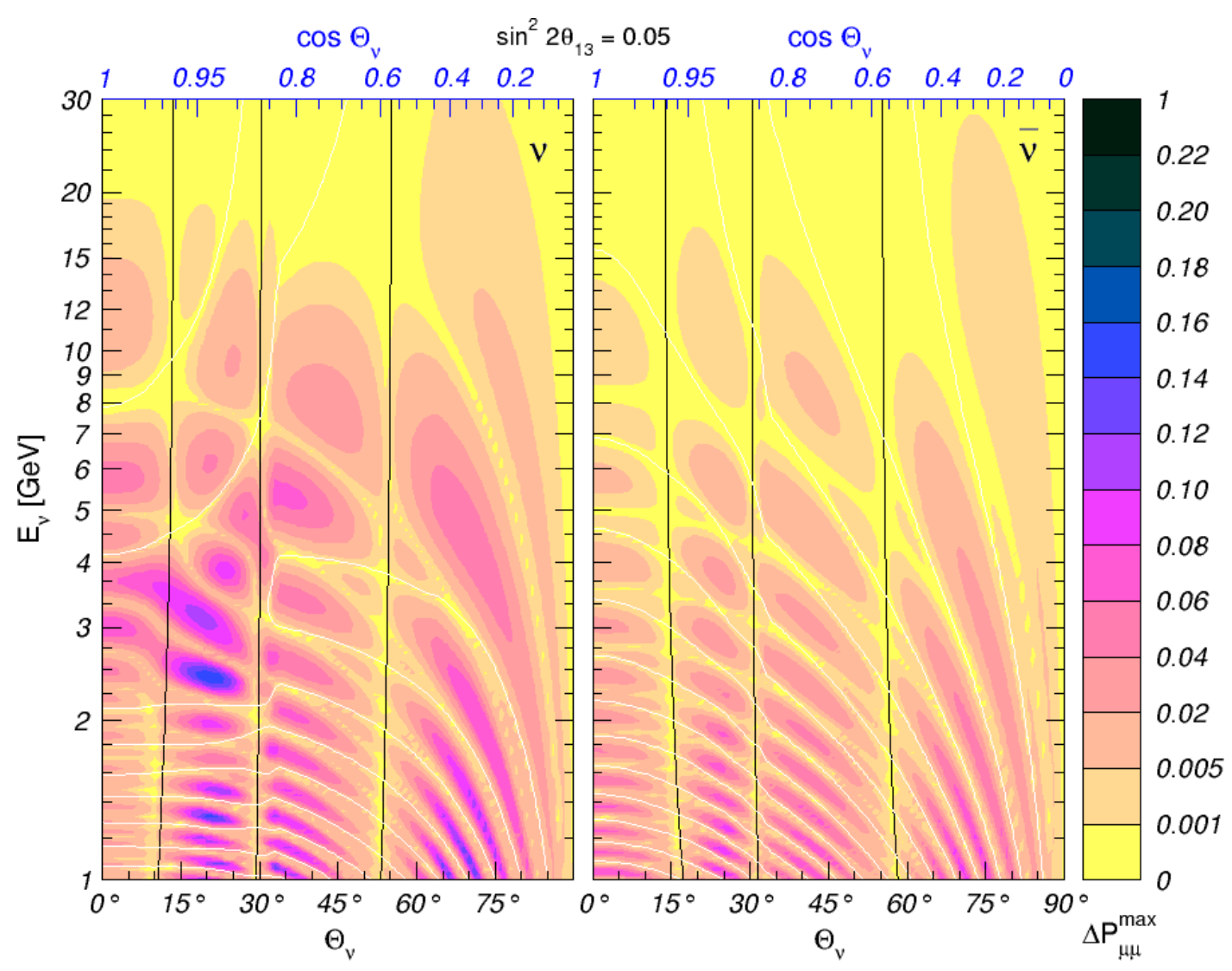

Figure 13: Contour plots for the probability difference $\Delta P_{\mu \mu}^{\max }=\max P_{\mu \mu}-\min P_{\mu \mu}$ for $\delta$ varying between $0^{\circ}$ and $360^{\circ}$ and all the other oscillation parameters fixed. Also shown are the solar (black) and atmospheric (white) magic curves. We set $\Delta m_{21}^{2}=8 \times 10^{-5} \mathrm{eV}^{2}$ and $\sin ^{2} 2 \theta_{13}=0.05$.

In the mantle domain the oscillation pattern consists of three MSW resonance peaks, which correspond to the oscillation phases $\pi / 2,3 \pi / 2$ and $5 \pi / 2$, and the parametric resonance ridge in the core domain at $E_{\nu} \approx 0.2 \mathrm{GeV}$ and $\Theta_{\nu} \sim 28-30^{\circ}$.

For non-zero 1-3 mixing the oscillograms consists of the low energy pattern, where the effect of the 1-2 mixing dominates, and the high energy pattern, determined mainly by the 1-3 mixing and mass splitting, if the 1-3 mixing is not too small. The low energy pattern is modulated by the high frequency and small amplitude effect induced by the 1-3 mode, whereas the high energy structure is modulated by the low (refraction) frequency small amplitude effect due to the 1-2 mass splitting and mixing.

We studied in detail the effect of the 1-2 mode on the oscillograms for energies $E_{\nu}>$ $1 \mathrm{GeV}$. At these energies, if $\theta_{13}$ is not very small, the oscillation pattern is determined mainly by the 1-3 mixing and mass splitting, whereas the 1-2 mass splitting and mixing lead to small corrections. In the $\nu_{e}-\nu_{e}$ channel the interference of the 1-2 and 1-3 modes is strongly suppressed, and the effect of the 1-2 mixing is due to corrections to the 1-3 mixing and the atmospheric phase. In the $\nu_{\mu}-\nu_{e}$ channel the effect of the 1-2 mixing is dominated by the interference of the solar and atmospheric amplitudes. These corrections have a domain structure in the $E_{\nu}-\Theta_{\nu}$ plane. In the $\nu_{\mu}-\nu_{\mu}$ and $\nu_{\mu}-\nu_{\tau}$ channels the effect 
of the 1-2 mixing is essentially due to the corrections to the phase of the main (vacuum) oscillation mode. This consideration is important in discussions of the degeneracies of parameters in terms of the oscillograms.

We studied the properties of the interference of the amplitudes $A_{e \tilde{2}}$ and $A_{e \tilde{3}}$ and, in particular, the effects of $\mathrm{CP}$-violation which are associated with this term. The structure of the interference term is simply and rather accurately determined in the factorization approximation, when one has $A_{e \tilde{3}} \approx A_{A}\left(\Delta m_{31}^{2}, \theta_{13}\right)$ and $A_{e \tilde{2}} \approx A_{S}\left(\Delta m_{21}^{2}, \theta_{12}\right)$. This means that the dependence of the parameters of 1-2 sector and 1-3 sector factorizes in the interference term. This approximation does not work in the resonance regions.

We showed that the interference term, and therefore CP-violation, exhibit a domain structure in the $E_{\nu}-\Theta_{\nu}$ plane. The borders of the domains are determined by the grids of magic lines (solar and atmospheric) and by the lines of the interference phase condition. In the neighboring domains the sign of the $\mathrm{CP}$ effect is opposite. Beyond the factorization approximation the interconnections of the solar and phase condition lines occur, which are related to the level crossing phenomenon.

We studied the dependence of the probabilities on the CP-phase. The character of the dependence on the CP-phase is different for survival and transition probabilities. In the standard parametrization, the $\nu_{e}$ survival probability does not depend on $\delta$. For the survival channels $\nu_{\mu}-\nu_{\mu}$ and $\nu_{\tau}-\nu_{\tau}$ as well as for the transition channel $\nu_{\mu}-\nu_{\tau}$ all three sets of the lines - the borders of the domains - do not depend on $\delta$. Within a given domain the interference term changes as $\propto \cos \delta$ in the $\nu_{\mu}-\nu_{\mu}$ channel, and as $\propto \sin \delta$ in the $\nu_{\mu}-\nu_{\tau}$ channel. For the probabilities of transitions which involve $\nu_{e}$, i.e. $\nu_{e}-\nu_{\mu}$ and $\nu_{e}-\nu_{\tau}$, the interference phase condition depends on $\delta$, so that with changing $\delta$ the corresponding lines move. Therefore, the modification of the pattern of $\mathrm{CP}$ violation is determined by this motion of the interference phase lines.

The phase $\delta$ can affect significantly all the oscillation probabilities but $\nu_{e} \leftrightarrow \nu_{e}$. Thus, in principle, one can study the effects of leptonic CP violation by precision measurements of the energy and zenith angle dependence of these probabilities. We find that the strongest variation of the probability with $\delta$ occurs in the region of the 1-2 resonance. For $\sin ^{2} 2 \theta_{13}<$ $1 / 9$ the local maximum appears in the 1-3 resonance region. The weakest variation is at $E_{\nu} \approx 0.5 E_{13}^{R}$.

Many features of the oscillograms discussed in this paper are unobservable in the present and forthcoming experiments. The accelerator experiments cover only several peripheral regions of the oscillograms, which correspond to large values of the nadir angles $\Theta_{\nu}>77^{\circ}$. The large underwater and ice detectors have high energy thresholds $E>15$ $\mathrm{GeV}$. Thus, the most interesting and structured regions of the oscillograms turn out to be uncovered. Measurements of the oscillograms with atmospheric neutrinos could be performed using multi-megaton water Cherenkov detectors, which will have sufficient statistics in the energy range $E>1-2 \mathrm{GeV}$, where the energy and angular resolutions are good enough $[69,70]$. Detailed consideration of various features of the oscillograms performed in this paper can help develop methods which will enhance the sensitivity of future experiments to the CP-violating phase and other neutrino parameters. 


\section{Acknowledgments}

A.Yu.S. is grateful to the Max-Planck-Institut für Kernphysik, Heidelberg, for hospitality. The work of A.Yu.S. has been supported in part by the Alexander von Humboldt Foundation. M.M. is supported by MCI through the Ramón y Cajal program and through the national project FPA2006-01105, and by the Comunidad Autónoma de Madrid through the HEPHACOS project P-ESP-00346.

\section{References}

[1] E. K. Akhmedov, M. Maltoni, and A. Y. Smirnov, 1-3 leptonic mixing and the neutrino oscillograms of the earth, JHEP 05 (2007) 077, [hep-ph/0612285].

[2] M. C. Gonzalez-Garcia and M. Maltoni, Phenomenology with massive neutrinos, Phys. Rept. (2008) in press, [arXiv:0704.1800].

[3] E. Lisi and D. Montanino, Earth regeneration effect in solar neutrino oscillations: An analytic approach, Phys. Rev. D56 (1997) 1792-1803, [hep-ph/9702343].

[4] Q. Y. Liu and A. Y. Smirnov, Neutrino mass spectrum with $\nu_{\mu} \rightarrow \nu_{s}$ oscillations of atmospheric neutrinos, Nucl. Phys. B524 (1998) 505-523, [hep-ph/9712493].

[5] Q. Y. Liu, S. P. Mikheyev, and A. Y. Smirnov, Parametric resonance in oscillations of atmospheric neutrinos?, Phys. Lett. B440 (1998) 319-326, [hep-ph/9803415].

[6] A. De Rujula, M. B. Gavela, and P. Hernandez, Neutrino oscillation physics with a neutrino factory, Nucl. Phys. B547 (1999) 21-38, [hep-ph/9811390].

[7] M. Freund, M. Lindner, S. T. Petcov, and A. Romanino, Testing matter effects in very long baseline neutrino oscillation experiments, Nucl. Phys. B578 (2000) 27-57, [hep-ph/9912457].

[8] M. Freund and T. Ohlsson, Matter enhanced neutrino oscillations with a realistic earth density profile, Mod. Phys. Lett. A15 (2000) 867-874, [hep-ph/9909501].

[9] E. K. Akhmedov, Matter effects in short-baseline neutrino oscillations, Phys. Lett. B503 (2001) 133-139, [hep-ph/0011136].

[10] I. Mocioiu and R. Shrock, Matter effects on neutrino oscillations in long baseline experiments, Phys. Rev. D62 (2000) 053017, [hep-ph/0002149].

[11] E. K. Akhmedov, P. Huber, M. Lindner, and T. Ohlsson, T violation in neutrino oscillations in matter, Nucl. Phys. B608 (2001) 394-422, [hep-ph/0105029].

[12] M. C. Banuls, G. Barenboim, and J. Bernabeu, Medium effects for terrestrial and atmospheric neutrino oscillations, Phys. Lett. B513 (2001) 391-400, [hep-ph/0102184].

[13] D. Indumathi and M. V. N. Murthy, A question of hierarchy: Matter effects with atmospheric neutrinos and anti-neutrinos, Phys. Rev. D71 (2005) 013001, [hep-ph/0407336].

[14] S. Palomares-Ruiz and S. T. Petcov, Three-neutrino oscillations of atmospheric neutrinos, $\theta_{13}$, neutrino mass hierarchy and iron magnetized detectors, Nucl. Phys. B712 (2005) 392-410, [hep-ph/0406096].

[15] R. Gandhi, P. Ghoshal, S. Goswami, P. Mehta, and S. Uma Sankar, Earth matter effects at very long baselines and the neutrino mass hierarchy, Phys. Rev. D73 (2006) 053001, [hep-ph/0411252]. 
[16] R. Gandhi, P. Ghoshal, S. Goswami, P. Mehta, and S. Uma Sankar, Large matter effects in $\nu_{\mu} \rightarrow \nu_{\tau}$ oscillations, Phys. Rev. Lett. 94 (2005) 051801, [hep-ph/0408361].

[17] K. Kimura, A. Takamura, and H. Yokomakura, Enhancement of cp violating terms for neutrino oscillation in earth matter, Phys. Lett. B600 (2004) 91-103, [hep-ph/0407126].

[18] G.-L. Lin and Y. Umeda, The matter effects to neutrino oscillations $\nu_{\mu} \rightarrow \nu_{e}, \nu_{\mu}$ at very long baselines and the neutrino mixing angles $\theta_{13}$ and $\theta_{23}$, hep-ph/0505009.

[19] E. K. Akhmedov, M. Maltoni, and A. Y. Smirnov, Oscillations of high energy neutrinos in matter: Precise formalism and parametric resonance, Phys. Rev. Lett. 95 (2005) 211801, [hep-ph/0506064].

[20] A. N. Ioannisian and A. Y. Smirnov, Describing neutrino oscillations in matter with magnus expansion, arXiv:0803.1967.

[21] A. D. Supanitsky, J. C. D'Olivo, and G. Medina-Tanco, Perturbative exponential expansion and matter neutrino oscillations, arXiv:0804.1105.

[22] M. Chizhov, M. Maris, and S. T. Petcov, On the oscillation length resonance in the transitions of solar and atmospheric neutrinos crossing the earth core, hep-ph/9810501.

[23] T. Ohlsson and H. Snellman, Neutrino oscillations with three flavors in matter: Applications to neutrinos traversing the earth, Phys. Lett. B474 (2000) 153-162, [hep-ph/9912295].

[24] M. Jacobson and T. Ohlsson, Extrinsic cpt violation in neutrino oscillations in matter, Phys. Rev. D69 (2004) 013003, [hep-ph/0305064].

[25] T. Kajita, Atmospheric neutrinos, New J. Phys. 6 (2004) 194.

[26] L. Wolfenstein, Neutrino oscillations in matter, Phys. Rev. D17 (1978) 2369.

[27] S. P. Mikheev and A. Y. Smirnov, Resonance enhancement of oscillations in matter and solar neutrino spectroscopy, Sov. J. Nucl. Phys. 42 (1985) 913-917.

[28] V. K. Ermilova, V. A. Tsarev, and V. A. Chechin, Parametric enhancement of neutrino oscillations in matter, Kr. Soob. Fiz. 5 (1986) 26. Short Notices of the Lebedev Institute.

[29] E. K. Akhmedov, On neutrino oscillations in a nonhomogeneous medium, Sov. J. Nucl. Phys. 47 (1988) 301-302.

[30] S. T. Petcov, Diffractive-like (or parametric-resonance-like?) enhancement of the earth (day-night) effect for solar neutrinos crossing the earth core, Phys. Lett. B434 (1998) 321-332, [hep-ph/9805262].

[31] E. K. Akhmedov, Parametric resonance of neutrino oscillations and passage of solar and atmospheric neutrinos through the earth, Nucl. Phys. B538 (1999) 25-51, [hep-ph/9805272].

[32] E. K. Akhmedov, A. Dighe, P. Lipari, and A. Y. Smirnov, Atmospheric neutrinos at super-kamiokande and parametric resonance in neutrino oscillations, Nucl. Phys. B542 (1999) 3-30, [hep-ph/9808270].

[33] M. V. Chizhov and S. T. Petcov, Enhancing mechanisms of neutrino transitions in a medium of nonperiodic constant-density layers and in the earth, Phys. Rev. D63 (2001) 073003, [hep-ph/9903424].

[34] T.-K. Kuo and J. T. Pantaleone, T nonconservation in three neutrino oscillations, Phys. Lett. B198 (1987) 406. 
[35] H. Minakata and H. Nunokawa, Cp violation vs. matter effect in long-baseline neutrino oscillation experiments, Phys. Rev. D57 (1998) 4403-4417, [hep-ph/9705208].

[36] K. Dick, M. Freund, M. Lindner, and A. Romanino, Cp-violation in neutrino oscillations, Nucl. Phys. B562 (1999) 29-56, [hep-ph/9903308].

[37] O. L. G. Peres and A. Y. Smirnov, Testing the solar neutrino conversion with atmospheric neutrinos, Phys. Lett. B456 (1999) 204-213, [hep-ph/9902312].

[38] H. Minakata and S. Watanabe, Solar neutrinos and leptonic cp violation, Phys. Lett. B468 (1999) 256-260, [hep-ph/9906530].

[39] T. Ota and J. Sato, Matter profile effect in neutrino factory, Phys. Rev. D63 (2001) 093004, [hep-ph/0011234].

[40] S. J. Parke and T. J. Weiler, Optimizing t-violating effects for neutrino oscillations in matter, Phys. Lett. B501 (2001) 106-114, [hep-ph/0011247].

[41] I. Mocioiu and R. Shrock, Neutrino oscillations with two $\delta m^{2}$ scales, JHEP 11 (2001) 050, [hep-ph/0106139].

[42] M. Freund, Analytic approximations for three neutrino oscillation parameters and probabilities in matter, Phys. Rev. D64 (2001) 053003, [hep-ph/0103300].

[43] H. Yokomakura, K. Kimura, and A. Takamura, Overall feature of cp dependence for neutrino oscillation probability in arbitrary matter profile, Phys. Lett. B544 (2002) 286-294, [hep-ph/0207174].

[44] M. C. Gonzalez-Garcia and M. Maltoni, Two mass-scale oscillation analysis of atmospheric and reactor data, Eur. Phys. J. C26 (2003) 417-428, [hep-ph/0202218].

[45] B. Brahmachari, S. Choubey, and P. Roy, Cp violation and matter effect for a variable earth density in very long baseline experiments, Nucl. Phys. B671 (2003) 483-497, [hep-ph/0303078].

[46] O. L. G. Peres and A. Y. Smirnov, Atmospheric neutrinos: Lma oscillations, $u_{e 3}$ induced interference and cp-violation, Nucl. Phys. B680 (2004) 479-509, [hep-ph/0309312].

[47] E. K. Akhmedov, R. Johansson, M. Lindner, T. Ohlsson, and T. Schwetz, Series expansions for three-flavor neutrino oscillation probabilities in matter, JHEP 04 (2004) 078, [hep-ph/0402175].

[48] H. Minakata, M. Sonoyama, and H. Sugiyama, Determination of $\theta_{23}$ in long-baseline neutrino oscillation experiments with three-flavor mixing effects, Phys. Rev. D70 (2004) 113012, [hep-ph/0406073].

[49] M. C. Gonzalez-Garcia, M. Maltoni, and A. Y. Smirnov, Measuring the deviation of the 2-3 lepton mixing from maximal with atmospheric neutrinos, Phys. Rev. D70 (2004) 093005, [hep-ph/0408170].

[50] A. Takamura and K. Kimura, Large non-perturbative effects of small $\delta m_{21}^{2} / \delta m_{31}^{2}$ and $\sin \theta_{13}$ on neutrino oscillation and cp violation in matter, JHEP 01 (2006) 053, [hep-ph/0506112].

[51] K. Kimura, A. Takamura, and T. Yoshikawa, Maximal cp phase effect and $\theta_{13}$ screening in long baseline neutrino experiments, hep-ph/0603141.

[52] K. Kimura, A. Takamura, and T. Yoshikawa, Measurement of leptonic cp phase in $\nu_{\mu} \rightarrow \nu_{\mu}$ oscillation, hep-ph/0605308. 
[53] W. Liao, Precise formulation of neutrino oscillation in the earth, Phys. Rev. D77 (2008) 053002, [arXiv:0710.1492].

[54] K. Kimura, A. Takamura, and T. Yoshikawa, Effect of leptonic cp phase in $\nu_{\mu} \rightarrow \nu_{\mu}$ oscillations, JHEP 03 (2008) 016, [arXiv:0711.1567].

[55] K. Kimura, A. Takamura, and T. Yoshikawa, Avoiding the uncertainty from correlation between $\left|\delta m_{31}^{2}\right|$ and cp phase delta in $\nu_{\mu} \rightarrow \nu_{\mu}$ long baseline experiments, arXiv:0803.0787.

[56] S. Toshev, On t violation in matter neutrino oscillations, Mod. Phys. Lett. A6 (1991) $455-460$.

[57] A. M. Dziewonski and D. L. Anderson, Preliminary reference earth model, Phys. Earth Planet. Interiors 25 (1981) 297-356.

[58] M. Maltoni, T. Schwetz, M. A. Tortola, and J. W. F. Valle, Status of global fits to neutrino oscillations, New J. Phys. 6 (2004) 122, [hep-ph/0405172].

[59] G. L. Fogli, E. Lisi, A. Marrone, and A. Palazzo, Global analysis of three-flavor neutrino masses and mixings, Prog. Part. Nucl. Phys. 57 (2006) 742-795, [hep-ph/0506083].

[60] A. Strumia and F. Vissani, Neutrino masses and mixings and..., hep-ph/0606054.

[61] A. Y. Smirnov, Neutrino oscillations: What is magic about the 'magic' baseline?, hep-ph/0610198.

[62] V. Barger, D. Marfatia, and K. Whisnant, Breaking eight-fold degeneracies in neutrino cp violation, mixing, and mass hierarchy, Phys. Rev. D65 (2002) 073023, [hep-ph/0112119].

[63] P. Huber, Cp, t and cpt violation in future long baseline experiments, J. Phys. G29 (2003) 1853-1856, [hep-ph/0210140].

[64] P. Huber and W. Winter, Neutrino factories and the 'magic' baseline, Phys. Rev. D68 (2003) 037301, [hep-ph/0301257].

[65] A. Blondel et al., Future neutrino oscillation facilities, Acta Phys. Polon. B37 (2006) 2077-2113, [hep-ph/0606111].

[66] P. Huber, M. Lindner, M. Rolinec, and W. Winter, Optimization of a neutrino factory oscillation experiment, Phys. Rev. D74 (2006) 073003, [hep-ph/0606119].

[67] S. K. Agarwalla, S. Choubey, and A. Raychaudhuri, Unraveling neutrino parameters with a magical beta-beam experiment at ino, arXiv:0711.1459.

[68] K. Kimura, A. Takamura, and T. Yoshikawa, New index of CP phase effect and $\theta_{13}$ screening in long baseline neutrino experiments, Phys. Lett. B640 (2006) 32-36.

[69] Y. Suzuki, An ultimate neutrino and proton decay detector: TITAND, in Proceedings of the 3rd NO-VE Workshop (M. Baldo Ceolin, ed.), pp. 239-246, Edizioni Papergraf, 2006.

[70] E. K. Akhmedov, M. Maltoni, and A. Y. Smirnov, in progress. 